\title{
WestVirginiaUniversity
}

THE RESEARCH REPOSITORY @ WVU

Graduate Theses, Dissertations, and Problem Reports

2012

\section{Cycles, Disjoint Spanning Trees and Orientations of Graphs}

Yanting Liang

West Virginia University

Follow this and additional works at: https://researchrepository.wvu.edu/etd

\section{Recommended Citation}

Liang, Yanting, "Cycles, Disjoint Spanning Trees and Orientations of Graphs" (2012). Graduate Theses, Dissertations, and Problem Reports. 3559.

https://researchrepository.wvu.edu/etd/3559

This Dissertation is protected by copyright and/or related rights. It has been brought to you by the The Research Repository @ WVU with permission from the rights-holder(s). You are free to use this Dissertation in any way that is permitted by the copyright and related rights legislation that applies to your use. For other uses you must obtain permission from the rights-holder(s) directly, unless additional rights are indicated by a Creative Commons license in the record and/ or on the work itself. This Dissertation has been accepted for inclusion in WVU Graduate Theses, Dissertations, and Problem Reports collection by an authorized administrator of The Research Repository @ WVU.

For more information, please contact researchrepository@mail.wvu.edu. 


\title{
Cycles, Disjoint Spanning Trees and Orientations of Graphs
}

\author{
Yanting Liang \\ Dissertation submitted to the \\ Eberly College of Arts and Sciences \\ at West Virginia University \\ in partial fulfillment of the requirements \\ for the degree of \\ Doctor of Philosophy \\ in \\ Mathematics \\ Adam Halasz, Ph.D., Co-Chair \\ Hong-Jian Lai, Ph.D., Co-Chair \\ Muhammad A. Choudhry, Ph.D. \\ John Goldwasser, Ph.D. \\ Jerzy Wojciechowski, Ph.D. \\ Cun-Quan Zhang, Ph.D. \\ Department of Mathematics
}

Keywords: degree sequence, hamiltonian-connected, line graphs, Supereulerian, edge-disjoint spanning trees, nowhere zero flows, $\bmod (2 p+1)$-orientations, stick-pulling problem

Copyright 2012 Yanting Liang 


\section{ABSTRACT \\ Cycles, Disjoint Spanning Trees and Orientations of Graphs}

\section{Yanting Liang}

A graph $G$ is hamiltonian-connected if any two of its vertices are connected by a Hamilton path (a path including every vertex of $G$ ); and $G$ is $s$-hamiltonian-connected if the deletion of any vertex subset with at most $s$ vertices results in a hamiltonianconnected graph. We prove that the line graph of a $(t+4)$-edge-connected graph is $(t+2)$-hamiltonian-connected if and only if it is $(t+5)$-connected, and for $s \geq 2$ every $(s+5)$-connected line graph is $s$-hamiltonian-connected.

For integers $l$ and $k$ with $l>0$, and $k \geq 0, C_{h}(l, k)$ denotes the collection of $h$-edgeconnected simple graphs $G$ on $n$ vertices such that for every edge-cut $X$ with $2 \leq|X| \leq 3$, each component of $G-X$ has at least $(n-k) / l$ vertices. We prove that for any integer $k>0$, there exists an integer $N=N(k)$ such that for any $n \geq N$, any graph $G \in C_{2}(6, k)$ on $n$ vertices is supereulerian if and only if $G$ cannot be contracted to a member in a well characterized family of graphs.

An orientation of an undirected graph $G$ is a $\bmod (2 p+1)$-orientation if under this orientation, the net out-degree at every vertex is congruence to zero $\bmod 2 p+1$. A graph $H$ is $\bmod (2 p+1)$-contractible if for any graph $G$ that contains $H$ as a subgraph, the contraction $G / H$ has a mod $(2 p+1)$-orientation if and only if $G$ has a $\bmod (2 p+1)$ orientation (thus every mod $(2 p+1)$-contractible graph has a mod $(2 p+1)$-orientation). Jaeger in 1984 conjectured that every $(4 p)$-edge-connected graph has a mod $(2 p+1)$ orientation. It has also been conjectured that every $(4 p+1)$-edge-connected graph is $\bmod (2 p+1)$-contractible. We investigate graphs that are mod $(2 p+1)$-contractible, and as applications, we prove that a complete graph $K_{m}$ is $(2 p+1)$-contractible if and only if $m \geq 4 p+1$; that every $(4 p-1)$-edge-connected $K_{4}$-minor free graph is $\bmod$ $(2 p+1)$-contractible, which is best possible in the sense that there are infinitely many $(4 p-2)$-edge-connected $K_{4}$-minor free graphs that are not mod $(2 p+1)$-contractible; and that every $(4 p)$-connected chordal graph is $\bmod (2 p+1)$-contractible. We also prove that 
the above conjectures on line graphs would imply the truth of the conjectures in general, and that if $G$ has a mod $(2 p+1)$-orientation and $\delta(G) \geq 4 p$, then $L(G)$ also has a mod $(2 p+1)$-orientation.

The design of an $n$ processor network with given number of connections from each processor and with a desirable strength of the network can be modelled as a degree sequence realization problem with certain desirable graphical properties. A nonincreasing sequence $d=\left(d_{1}, d_{2}, \cdots, d_{n}\right)$ is graphic if there is a simple graph $G$ with degree sequence $d$. It is proved that for a positive integer $k$, a graphic nonincreasing sequence $d$ has a simple realization $G$ which has $k$-edge-disjoint spanning trees if and only if either both $n=1$ and $d_{1}=0$, or $n \geq 2$ and both $d_{n} \geq k$ and $\sum_{i=1}^{n} d_{i} \geq 2 k(n-1)$.

We investigate the emergence of specialized groups in a swarm of robots, using a simplified version of the stick-pulling problem [56], where the basic task requires the collaboration of two robots in asymmetric roles. We expand our analytical model [57] and identify conditions for optimal performance for a swarm with any number of species. We then implement a distributed adaptation algorithm based on autonomous performance evaluation and parameter adjustment of individual agents. While this algorithm reliably reaches optimal performance, it leads to unbounded parameter distributions. Results are improved by the introduction of a direct parameter exchange mechanism between selected high- and low-performing agents. The emerging parameter distributions are bounded and fluctuate between tight unimodal and bimodal profiles. Both the unbounded optimal and the bounded bimodal distributions represent partitions of the swarm into two specialized groups. 


\section{Acknowledgements}

First and foremost, I am most indebted to my co-advisors, Dr. Hong-Jian Lai and Dr. Adam Halasz, for their continued encouragement and support over these last few years. It is a pleasure to work under their supervision. Without them, this dissertation could not have come about.

I would like to take this opportunity to thank Dr. Bolian Liu at South China Normal University, who brought me to the research field of combinatorics and graph theory.

I would also like to thank other committee members: Dr. Muhammad A. Choudhry, Dr. John Goldwasser, Dr. Jerzy Wojciechowski, and Dr. Cun-Quan Zhang, for their help during my studies.

And finally, I would like to thank the Department of Mathematics and Eberly College

of Arts and Sciences at West Virginia University for providing me with an excellent study environment and support during my study as a graduate student. 


\section{DEDICATION}

To

my father Zhaosen Liang , my mother Qiulian Zhang

and

my husband Haosheng Chen, my daughter Chloe L. Chen 


\section{Contents}

1 Preliminaries 1

1.1 Notation and Terminology . . . . . . . . . . . . . . . . . . 1

1.2 Catlin's Reduction Method . . . . . . . . . . . . . . . . . . 4

$1.3 \operatorname{Mod}(2 p+1)$-orientations $\ldots \ldots \ldots \ldots \ldots \ldots \ldots$

1.4 Main Results . . . . . . . . . . . . . . . . . . . . . . . . 8

2 Hamiltonian-connected Line Graphs $\quad 10$

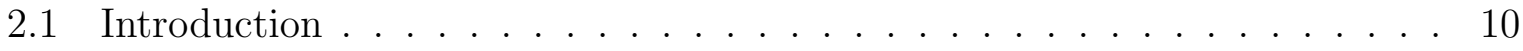

2.2 Proof of Theorems ?? and $? ? \ldots \ldots \ldots \ldots$

2.3 A Remark . . . . . . . . . . . . . . . . . . . . 16

3 Characterization of Supereulerian Graphs in $C_{2}(6, k) \quad 17$

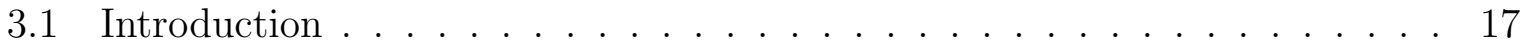

3.2 An Associate Result . . . . . . . . . . . . . . . . . . . . . . . . . . . 19

3.3 Proof of the Main Result . . . . . . . . . . . . . . . . . . . . . 34 
4 Characterization of Graphs with Mod $(2 p+1)$-orientations

4.1 Some Useful Facts . . . . . . . . . . . . . . . . . . . . . . . . . . 37

4.2 A Characterization of Graphs with $\operatorname{Mod}(2 p+1)$-orientations . . . . . . . 42

5 Characterization of Mod $(2 p+1)$-contractible Graphs 46

5.1 Proof of Theorem ?? . . . . . . . . . . . . . . . . . 46

6 Some Families of Graphs That Are Mod $(2 p+1)$-contractible $\quad 51$

6.1 Graphs without $K_{4}$-minors . . . . . . . . . . . . . . . . 51

6.2 Chordal Graphs . . . . . . . . . . . . . . . . . 52

$7 \operatorname{Mod}(2 p+1)$-orientation of Line Graphs $\quad 56$

7.1 Introduction . . . . . . . . . . . . . . . . 56

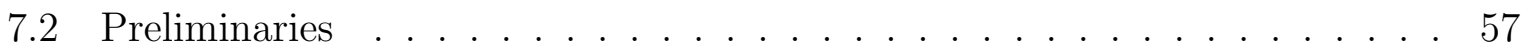

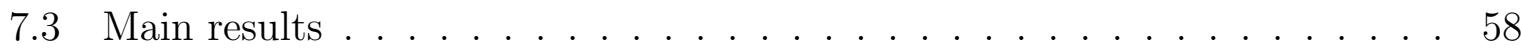

8 Graphs with Disjoint Spanning Trees $\quad 62$

8.1 Introduction . . . . . . . . . . . . . . . . . 62

8.2 Properties of Graphs with $k$ edge-disjoint Spanning Trees . . . . . . . . . . 63

8.3 Characterizations of Graphic Sequences with Realizations Having $k$ edgedisjoint Spanning Trees . . . . . . . . . . . . . . . . . . . . 69

9 Emergence of Specialization in a Swarm of Robots $\quad 74$ 
9.1 Introduction . . . . . . . . . . . . . . . . . . 74

9.2 Model and Analytical Results . . . . . . . . . . . . . . . . . . 76

9.2.1 The Stick Pulling Problem . . . . . . . . . . . . . . 76

9.2.2 Equations of Motion .................. 76

9.2.3 Steady state analysis . . . . . . . . . . . . 78

9.2.4 Pulling rates and optimality . . . . . . . . . . . . 79

9.3 Simulation Methods . . . . . . . . . . . . . . . . 82

9.3.1 Basic simulation algorithm . . . . . . . . . . . . . 82

9.3 .2 Individual adaptation . . . . . . . . . . . . . . 83

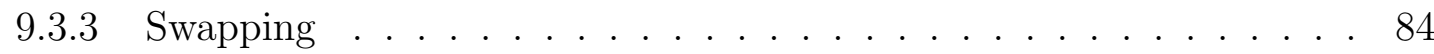

9.4 Results and Discussion . . . . . . . . . . . . . . . . 84

9.4.1 Model Validation - Equilibration ............. 84

9.4.2 Individual adaptation algorithm . . . . . . . . . . . 85

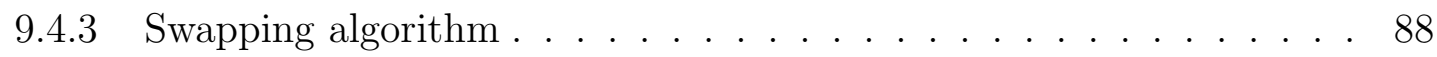

9.4.4 Waiting time parameter distributions . . . . . . . . . . . . 90

9.5 Summary and Conclusions . . . . . . . . . . . . . . . . . 90 


\section{Chapter 1}

\section{Preliminaries}

\section{$1.1 \quad$ Notation and Terminology}

We use [1] for terminology and notations not defined here. Let $G$ be a graph. We use $V(G)$ and $E(G)$ to denote the set of vertices and the set of edges of $G$, respectively. Two vertices $u, v$ are adjacent if $u v \in E(G)$. If any two vertices are adjacent in $G$, then $G$ is called a complete graph. A complete graph on $n$ vertices is denoted by $K_{n}$. A graph is bipartite if its vertex set can be partitioned into two subsets $X$ and $Y$ so that every edge has one end in $X$ and one end in $Y$; if every vertex in $X$ is joined to every vertex in $Y$, then $G$ is called a complete bipartite graph, denoted $K_{m, n}$ where $|X|=m$ and $|Y|=n$.

The number of edges incident with a vertex $v \in V(G)$ is called the degree of $v$ in $G$, and is denoted by $d_{G}(v)$ or $d(v)$. We use $\Delta(G)$ and $\delta(G)$ to denote the maximum and minimum degree of $G$, respectively. A graph with at least two vertices is called a nontrivial graph. For an integer $k>0$, a $k$-cycle, denoted by $C_{k}$, is a connected graph with $k$ vertices and in which each vertex has degree 2. A vertex cut of $G$ is a subset $V^{\prime}$ of $V(G)$ such that $G-V^{\prime}$ is disconnected. A $k$-vertex cut is a vertex cut of $k$ elements. A complete graph has no vertex cut; in fact, the only graphs that do not have vertex cuts are those that contain complete graphs as spanning subgraphs. If $G$ has at least one pair 
of distinct nonadjacent vertices, the connectivity $\kappa(G)$ of $G$ is the minimum $k$ for which $G$ has a $k$-vertex cut; otherwise, we define $\kappa(G)$ to be $|V(G)|-1$. A graph $G$ is said to be $k$-connected if $\kappa(G) \geq k$. Similarly, an edge cut of $G$ is a subset $E^{\prime}$ of $E(G)$ such that $G-E^{\prime}$ is disconnected. A $k$-edge cut is an edge cut of $k$ elements. We define the edge-connectivity $\kappa^{\prime}(G)$ of $G$ to be the minimum $k$ for which $G$ has a $k$-edge cut. A graph $G$ is said to be $k$-edge-connected if $\kappa^{\prime}(G) \geq k$. An edge $e=u v$ is called a pendant edge of graph $G$ if either $d_{G}(u)=1$ or $d_{G}(v)=1$. For a vertex or an edge subset $X$ of $G, G[X]$ denotes the subgraph of $G$ induced by $X$. For subsets $S, S^{\prime} \subseteq V(G)$, [S, $\left.S^{\prime}\right]$ denotes the set of edges of $G$ with one end in $S$ and the other in $S^{\prime}$.

For a graph $G$ and for $v \in V(G)$, the neighborhood $N_{G}(v)$ denotes the set of all vertices adjacent to $v$ in $G$ and $\tau(G)$ denotes the number of edge-disjoint spanning trees of $G$. .

For a graph $G$ and each $i=0,1,2, \cdots$, we let $d_{G}(v)$ denote the degree of $v$ in $G$ and $D_{i}(G)=\left\{v \in V(G) \mid d_{G}(v)=i\right\}$. Let $d_{i}(G)=\left|D_{i}(G)\right|$. When the graph $G$ is understood in the context, we use the following short-hand notations: $D_{i}=D_{i}(G), d(v)=d_{G}(v)$ and $d_{i}=d_{i}(G)$. Moreover, for an integer $k \geq 0$, a vertex of degree $k$ in a graph $G$ is sometimes referred as a $k$-vertex of $G$. If $A \subseteq V(G)$, we let $G-A=G[V(G)-A]$. When $A=\{v\}$, we use $G-v$ for $G-\{v\}$.

An edge cut $X$ of $G$ is essential if at least two components of $G-X$ are nontrivial. A graph $G$ is essentially $k$-edge-connected if $|E(G)| \geq k+1$ and if for every $E_{0} \subseteq E(G)$ with $\left|E_{0}\right|<k, G-E_{0}$ has exactly one component $H$ with $E(H) \neq \varnothing$.

Let $X \subseteq E(G)$. The contraction $G / X$ is the graph obtained from $G$ by identifying the two ends of each edge in $X$ and then deleting the resulting loops. For convenience, we use $G / e$ for $G /\{e\}$ and $G / \varnothing=G$; and if $H$ is a subgraph of $G$, we write $G / H$ for $G / E(H)$. Note that even if $G$ is a simple graph, contracting some edges of $G$ may result in a graph with multiple edges. If $K$ is a connected subgraph of $G$, and if $v_{K}$ is the vertex in $G / K$ onto which $K$ is contracted, then $K$ is called the pre-image of $v_{K}$.

The line graph of a graph $G$, denoted by $L(G)$, has $E(G)$ as its vertex set, where two vertices in $L(G)$ are adjacent if and only if the corresponding two edges in $G$ share 
one common end-vertex.

A cycle of a graph $G$ is called a hamiltonian cycle if it visits each vertex of $G$ exactly once. A path of a graph $G$ is called a hamiltonian path if it visits each vertex of $G$ exactly once. A graph $G$ is called hamiltonian if it has a hamiltonian cycle; $G$ is called hamiltonian-connected if for any two distinct vertices $u, v \in V(G), G$ has a hamiltonian path connecting $u$ and $v$; and $G$ is s-hamiltonian-connected if the deletion of any vertex subset with at most $s$ vertices results in a Hamiltonian-connected graph.

An Euler circuit is a closed walk that traverses each edge exactly once. A graph $G$ is called eulerian if it has an Euler circuit; $G$ is Supereulerian if it has a spanning eulerian subgraph. We denote by $\mathcal{S}$ the family of all Supereulerian graphs.

A subgraph $H$ of a graph $G$ is dominating if $G-V(H)$ is edgeless. Harary and Nash-Williams proved a very useful connection between hamiltonian cycles in the line grpah $L(G)$ and dominating eulerian subgraphs in $G$.

Theorem 1.1.1 (Harary and Nash-Willaims [6]) For a connected graph $G$ with $|E(G)| \geq$ $3, L(G)$ is hamiltonian if and only if $G$ has a dominating eulerian subgraph.

Let $e_{1}, e_{2} \in E(G)$. A trail in $G$ whose first edge is $e_{1}$ and last edge is $e_{2}$ is called an $\left(e_{1}, e_{2}\right)$-trail. Let $T$ be an $\left(e_{1}, e_{2}\right)$-trail. Then $T$ is dominating if every $e \in E(G)$ is incident with an internal vertex of $T$; and $T$ is spanning if $T$ is dominating and $V(T)=V(G)$. For $v_{1}, v_{2} \in V(G)$, a trail in $G$ whose origin is $v_{1}$ and terminus is $v_{2}$ is called a $\left(v_{1}, v_{2}\right)$-trail, and it is a spanning $\left(v_{1}, v_{2}\right)$-trail if it contains every vertex of $G$.

Theorem 1.1.2 (Catlin and Lai, Theorem 4 in [5]) Let $G$ be a graph and let $e_{1}, e_{2} \in$ $E(G)$. If $G$ has two edge-disjoint spanning trees, then exactly one of the following holds:

(a) G has a spanning $\left(e_{1}, e_{2}\right)$-trail;

(b) $\left\{e_{1}, e_{2}\right\}$ is an essential edge-cut of $G$.

With similar arguments in [6], the following is obtained. 
Theorem 1.1.3 Let $G$ be a connected graph with at least 3 edges. The line graph $L(G)$ is hamiltonian-connected if and only if for any $e_{1}, e_{2} \in E(G), G$ has a dominating $\left(e_{1}, e_{2}\right)$ trail.

\subsection{Catlin's Reduction Method}

A graph $G$ is collapsible if for any even subset $R \subseteq V(G), G$ has a spanning connected subgraph $H$ such that $O(H)=R$ where $O(H)$ denotes the set of odd vertices of $H$. The reduction of $G$ is the graph obtained from $G$ by contracting each maximal collapsible subgraph of $G$ to a distinct vertex. If $G$ is the reduction of itself, then $G$ is reduced.

By definition, the 3 -cycle $C_{3}$ is collapsible, and any collapsible graph is supereulerian.

Theorem 1.2.1 (Li, Lai and Zhan, Lemma 2.2 in [8]) If $G$ is collapsible, then $\forall x, y \in$ $V(G)$, there exists a $(x, y)$-trail $T$ of $G$ such that $V(T)=V(G)$.

Define $F(G)$ to be the minimum number of edges that must be added to $G$ so that the resulting graph has two edge-disjoint spanning trees. The edge arboricity $a(G)$ of a graph $G$ is the minimum number of forests in $G$ whose union contains $G$. Nash-Williams [25] proved

$$
a(G)=\max _{H \subseteq G}\left\lceil\frac{|E(H)|}{|V(H)|-1}\right\rceil .
$$

Theorem 1.2.2 (Catlin) Let $G$ be a graph.

(i) (Theorem 2 in [2]) If $F(G)=0$, then $G$ is collapsible.

(ii) (Theorem 3 in [2]) If $H$ is a collapsible subgraph of $G$, then $G \in \mathcal{S}$ if and only if $G / H \in \mathcal{S}$.

(iii) (Theorem 8(iv) in [2]) If $H$ is a collapsible subgraph of $G$, then $G$ is collapsible if and only if $G / H$ is collapsible.

(iv) (Theorem 5 and 8(iii) in [2]) If $G$ is reduced, then any subgraph of $G$ is reduced and $a(G) \leq 2$. 
(v) (Theorem 8(iv) in [2]) If $a(G) \leq 2$, then $F(G)=2|V(G)|-|E(G)|-2$. In particular, if $G$ is a reduced graph, then $F(G)=2|V(G)|-|E(G)|-2$.

(vi) (Lemma 1 in [15]) For any $e \in E\left(K_{3,3}\right), K_{3,3}-e$ is collapsible.

Theorem 1.2.3 (Catlin et al, Theorem 6 in [17]) For a graph $G$, if $\max _{K \subseteq G} \frac{|E(K)|}{|V(K)|-1} \geq 2$, then $G$ has a nontrivial induced subgraph $H$ that has two edge-disjoint spanning trees, i.e. $F(H)=0$.

The following corollary follows from the theorems above directly:

Corollary 1.2.4 If $G$ is reduced, then $|E(H)| /(|V(H)|-1)<2$ for any nontrivial induced subgraph $H$ of $G$.

Proof. By Theorem 1.2.2(iv) and equation (1), $|E(H)| /(|V(H)|-1) \leq 2$ for any nontrivial induced subgraph $H$ of $G$. Assume there exists $H$ such that $|E(H)| /(|V(H)|-1)=2$. Then by Theorem 1.2.3 and 1.2.2(i), $G$ has a nontrivial collapsible subgraph, contrary to that $G$ is reduced. Hence, $|E(H)| /(|V(H)|-1)<2$.

Theorem 1.2.5 (Catlin, Theorem 7 in [2]) If $F(G) \leq 1$, then $G$ is collapsible if and only if $\kappa^{\prime}(G) \geq 2$.

Theorem 1.2.6 (Catlin et al, Theorem 1.3 in [4]) If $G$ is connected and if $F(G) \leq 2, G$ is collapsible if and only if the reduction of $G$ is not a $K_{2}$ or $K_{2, t}$ with $t \geq 1$.

Theorem 1.2.7 (Catlin, Theorem 8 and Lemma 5 of [2]) If $G$ is reduced, then $G$ is simple and has no $K_{3}$. Moreover, if $\kappa^{\prime}(G) \geq 2$, then $\sum_{i=2}^{3}\left|D_{i}(G)\right| \geq 4$, and when $\sum_{i=2}^{3}\left|D_{i}(G)\right|=4$, $G$ must be Eulerian. 


\section{$1.3 \quad \operatorname{Mod}(2 p+1)$-orientations}

Let $\mathbb{Z}$ denotes the set of all integers. For an $m \in \mathbb{Z}, \mathbb{Z}_{m}$ denotes the set of integers modulo $m$, as well as the additive cyclic group on $m$ elements.

Let $D=D(G)$ be an orientation of an undirected graph $G$. If an edge $e \in E(G)$ is directed from a vertex $u$ to a vertex $v$, then define $\operatorname{tail}(e)=u$ and $h e a d(e)=v$. For vertex sets $U, V \subset V(G)$ with $U \cap V=\varnothing$, denote

$$
\begin{gathered}
E_{G}(U, V)=\{u v \in E(G): u \in U, v \in V\}, \\
E_{D}^{-}(U, V)=\{e=u v \in E(D): \operatorname{head}(e)=u \in U, \operatorname{tail}(e)=v \in V\}, \\
E_{D}^{+}(U, V)=\{e=u v \in E(D): \operatorname{tail}(e)=u \in U, \operatorname{head}(e)=v \in V\} .
\end{gathered}
$$

Let $d_{D}^{-}(U, V)=\left|E_{D}^{-}(U, V)\right|$ and $d_{D}^{+}(U, V)=\left|E_{D}^{+}(U, V)\right|$. If $U=\{v\}$ and $V=V(G)-\{v\}$, then we use $E_{G}(v), E_{D}^{-}(v)$ and $E_{D}^{+}(v)$ to denote the subsets of edges incident with $v$ in $G$, directed into $v$ and directed from $v$ under orientation $D$, respectively, and let $d_{D}^{-}(v)=\left|E_{D}^{-}(v)\right|$ and $d_{D}^{+}(v)=\left|E_{D}^{+}(v)\right|$. The subscript $D$ may be omitted when $D(G)$ is understood from the context.

For a function $f: E \mapsto \mathbf{Z}_{m}$, define the boundary of $f \partial f: V(G) \mapsto \mathbb{Z}_{m}$ as follows:

$$
\partial f(v) \equiv \sum_{e \in E_{D}^{+}(v)} f(e)-\sum_{e \in E_{D}^{-}(v)} f(e)(\bmod m) .
$$

A function $b: V(G) \mapsto \mathbf{Z}_{m}$ is a zero sum function on $\mathbb{Z}_{m}$ if $\sum_{v \in V(G)} b(v) \equiv 0(\bmod m)$. The set of all zero sum functions on $\mathbb{Z}_{m}$ of $G$ is denoted by $Z\left(G, \mathbb{Z}_{m}\right)$. When $m=2 p+1>0$ is an odd number, we define $M_{2 p+1}^{o}$ to be the collection of graphs such that $G \in M_{2 p+1}^{o}$ if and only if $\forall b \in Z\left(G, \mathbb{Z}_{2 p+1}\right), \exists f: E(G) \mapsto\{1,-1\}$ such that $\forall v \in V(G), \partial f(v) \equiv b(v)$ $(\bmod 2 p+1)$.

For a graph $G$, if $G$ has an orientation $D$ such that at every vertex $v \in V(G)$, $d_{D}^{+}(v)-d_{D}^{-}(v) \equiv 0(\bmod 2 p+1)$, then we say that $G$ admits a $\bmod (2 p+1)$-orientation. The set of all graphs which have $\bmod (2 p+1)$-orientations is denoted by $\mathbf{M}_{\mathbf{2} \mathbf{p}+\mathbf{1}}$. It can be proved that $G$ has a 3 -NZF if and only if $G \in M_{3}$ by letting $f: E \mapsto\{1,-1\}$ with 
$\partial f=0$ and then reversing the orientation of $e$ for every $e \in E(G)$ with $f(e)=-1$ to obtain a mod 3-orientation. As implied in [37], the property for $G$ to be in $M_{2 p+1}$ or in $M_{2 p+1}^{o}$ is independent of the orientation $D(G)$.

Tutte and Jeager proposed the following conjectures concerning the membership of $M_{2 p+1}$. A conjecture concerning the membership in $M_{2 p+1}^{o}$ is also proposed recently.

Conjecture 1.3.1 Let $p \geq 1$ denote an integer.

(i) (Tutte, [40]) Every 4-edge-connected graph is in $M_{3}$.

(ii) (Jaeger, [32] and [33]) Every (4p)-edge-connected graph is in $M_{2 p+1}$.

(iii) ([37] and [38]) Every $(4 p+1)$-edge-connected graph is in $M_{2 p+1}^{o}$

Conjecture 1.3.1 (i) is well-known as Tutte's 3-flow conjecture. Conjecture 1.3.1 (ii) is an extension of Tutte's 3-flow conjecture, which includes Conjecture 1.3.1 (i) as the special case of $p=1$. To the best of our knowledge, all these conjectures remain open.

Given a zero sum function $b \in Z\left(G, \mathbf{Z}_{2 p+1}\right)$, and a map $f: E(G) \mapsto\{1,-1\}$ with $\partial f \equiv b$ in $\mathbf{Z}_{2 p+1}$ (under the current orientation $D$ of $G$ ), one can reverse the orientation of $e$ for each $e \in E(G)$ with $f(e)=-1$ to obtain an orientation $D^{\prime}$ of $G$ such that $\forall v \in V(G)$, $d_{D^{\prime}}^{+}(v)-d_{D^{\prime}}^{-}(v)=\partial f(v)$. This orientation will be called a $\left(\mathbb{Z}_{2 p+1}, b\right)$-orientation of $G$. Thus we have the following propositions.

Proposition 1.3.2 ([37]) Let $G$ be a graph. Then $G \in M_{2 p+1}^{o}$ if and only if $\forall b \in$ $Z\left(G, \mathbb{Z}_{2 p+1}\right), G$ has a $\left(\mathbb{Z}_{2 p+1}, b\right)$-orientation, that is, an orientation $D$ with the property that $\forall v \in V(G), d_{D}^{+}(v)-d_{D}^{-}(v) \equiv b(v)(\bmod 2 p+1)$.

Proposition 1.3.3 ([37]) For any integer $p \geq 1$, each of the following holds.

(C1) $K_{1} \in M_{2 p+1}^{o}$.

(C2) If $e \in E(G)$ and if $G \in M_{2 p+1}^{o}$, then $G / e \in M_{2 p+1}^{o}$.

(C3) If $H$ is a subgraph of $G$ and $H, G / H \in M_{2 p+1}^{o}$, then $G \in M_{2 p+1}^{o}$.

(C2') If $e \in E(G)$ and if $G \in M_{2 p+1}$, then $G / e \in M_{2 p+1}$.

(C3') If $H$ is a subgraph of $G$ with $H \in M_{2 p+1}^{o}$, then $G / H \in M_{2 p+1}$ if and only if $G \in M_{2 p+1}$. 
Proof. Statements (C1)-(C3) can be found in Proposition 2.2 of [37]. The proofs of $\left(\mathrm{C} 2{ }^{\prime}\right)$ and $\left(\mathrm{C} 3{ }^{\prime}\right)$ are similar to those for $(\mathrm{C} 2)$ and $(\mathrm{C} 3)$, respectively, and so they are omitted.

\subsection{Main Results}

In the coming several chapters, we will present the following main results in this dissertation.

(1) The line graph of a $(t+4)$-edge-connected graph is $(t+2)$-hamiltonian-connected if and only if it is $(t+5)$-connected, and for $s \geq 2$ every $(s+5)$-connected line graph is $s$-hamiltonian-connected.

(2) For integers $h, l$ and $k$ with $h, l>0$, and $k \geq 0, C_{h}(l, k)$ denotes the collection of $h$-edge-connected simple graphs $G$ on $n$ vertices such that for every edge-cut $X$ with $2 \leq|X| \leq 3$, each component of $G-X$ has at least $(n-k) / l$ vertices. We prove that for any integer $k>0$, there exists an integer $N=N(k)$ such that for any $n \geq N$, any graph $G \in C_{2}(6, k)$ on $n$ vertices is supereulerian if and only if $G$ cannot be contracted to a member in a well characterized family of graphs.

(3) Let $G$ be a connected graph. Then $G$ has a mod $(2 p+1)$-orientation if and only if $G$ is the contraction of a $(2 p+1)$-regular bipartite graph.

(4) We show that $M_{2 p+1}^{o}$ consists of exactly the graphs $H$ with the following property: for any graph $G$ which contains $H$ as a subgraph, $G \in M_{2 p+1}$ if and only if $G / H \in M_{2 p+1}$.

(5) Every $(4 p-1)$-edge-connected $K_{4}$-minor free graph is mod $(2 p+1)$-contractible, and that every $(4 p)$-connected chordal graph is mod $(2 p+1)$-contractible. Both edgeconnectivity conditions are best possible.

(6) Jaeger in 1984 conjectured that every (4p)-edge-connected graph has a mod $(2 p+1)$-orientation. It has also been conjectured that every $(4 p+1)$-edge-connected graph is mod $(2 p+1)$-contractible. We prove that the above conjectures on line graphs 
would imply the truth of the conjectures in general, and we also prove that if $G$ has a $\bmod (2 p+1)$-orientation and $\delta(G) \geq 4 p$, then $L(G)$ also has a mod $(2 p+1)$-orientation.

(7) For a positive integer $k$, a graphic nonincreasing sequence $d$ has a simple realization $G$ which has $k$-edge-disjoint spanning trees if and only if either both $n=1$ and $d_{1}=0$, or $n \geq 2$ and both $d_{n} \geq k$ and $\sum_{i=1}^{n} d_{i} \geq 2 k(n-1)$. 


\section{Chapter 2}

\section{Hamiltonian-connected Line Graphs}

\section{$2.1 \quad$ Introduction}

It is well known that high connectivity does not assure the existence of a hamiltonian cycle, as evidenced by the complete bipartite graph $K_{m+1, m}$ for large $m$. However, for a line graph, C. Thomassen [9] made the following conjecture.

Conjecture 2.1.1 (Thomassen [9]) Every 4-connected line graph is hamiltonian.

Dr. Zhan made the following progresses towards Thomassen's Conjecture.

Theorem 2.1.2 (Zhan, Theorem 3 in [10]) If $\kappa^{\prime}(G) \geq 4$, then $L(G)$ is hamiltonianconnected.

Theorem 2.1.3 (Zhan, Theorem 3 in [11]) If $\kappa(L(G)) \geq 7, L(G)$ is hamiltonian-connected.

The main purpose of chapter is to sharpen both theorems obtained by Zhan. In fact, we proved the following two theorems in this note, for integers $t \geq 0$ and $s \geq 2$. 
Theorem 2.1.4 The line graph of a $(t+4)$-edge-connected graph is $(t+2)$-hamiltonianconnected if and only if it is $(t+5)$-connected.

Theorem 2.1.5 Every $(s+5)$-connected line graph is s-hamiltonian-connected.

\subsection{Proof of Theorems 2.1.4 and 2.1.5}

Throughout this section, we assume $t \geq 0$ and $s \geq 2$ are integers. First, we review some of mechanisms needed in the arguments.

Theorem 2.2.1 (Zhan, Corollary 10 in [11]) Let $G$ be a graph with $\kappa^{\prime}(G) \geq 3$ and $\kappa(L(G)) \geq 7$. Then for every pair $x$ and $y$ of edges of $G$, the subgraph $G-\{x, y\}$, or $G-\{x\}$ if $x$ and $y$ have an end-vertex of degree 3 in common, can be decomposed into two connected factors $F_{1}$ and $F_{2}$.

The core of a graph $G$, denoted by $G_{o}$, is obtained by deleting all vertices of degree 1 and contracting exactly one edge of $x y$ or $y z$ for each path $x y z$ in $G$ with $d(y)=2$. This notation is used throughout this chapter.

By the definition of the core graph $G_{o}$, all vertices of degree one or two are deleted or contracted and so $\delta\left(G_{o}\right) \geq 3$. Note that an essential edge cut of $G$ corresponds to a vertex cut of $L(G)$ and vice versa. So if $\kappa(L(G)) \geq 7$, then $\kappa^{\prime}\left(G_{o}\right) \geq 3$ and $\kappa\left(L\left(G_{o}\right)\right) \geq 7$. The following is also useful.

Theorem 2.2.2 (Catlin, Theorem 2 in [3]) Let $G$ be a connected graph and let $k \geq 1$ be an integer, then $\kappa^{\prime}(G) \geq 2 k$ if and only if $\forall X \subseteq E(G)$ with $|X| \leq k, \tau(G-X) \geq k$.

\section{Proof of Theorem 2.1.4}

Note that $K_{4}-e$ (where $e$ is an edge of a complete graph $K_{4}$ ) is 2-connected, but not hamiltonian-connected. So a hamiltonian-connected graph is 3 -connected and an 
$s$-hamiltonian-connected graph is $(s+3)$-connected. Thus if $L(G)$ is $(t+2)$-hamiltonianconnected, then $\kappa(L(G)) \geq t+5$. It suffices to prove that if $\kappa(L(G)) \geq t+5$, then $L(G)$ is $(t+2)$-hamiltonian-connected.

To show that $L(G)$ is $(t+2)$-hamiltonian connected, it suffices to show that $\forall Y \subseteq$ $V(L(G))=E(G)$, with $|Y| \leq t+2$ and $\forall e_{1}, e_{2} \in E(G)-Y, L(G)-Y$ has a hamiltonian $\left(e_{1}, e_{2}\right)$-path. By Theorem 1.1.3, this amounts to show that $G-Y$ has a dominating $\left(e_{1}, e_{2}\right)$-trail.

Since $|Y| \leq t+2$, we can choose a subset $Y_{1} \subseteq Y$, and let $Y_{2}=Y-Y_{1}$, such that $\left|Y_{1}\right| \leq t$ and $\left|Y_{2}\right| \leq 2$. Since $\kappa^{\prime}(G) \geq 4, \kappa^{\prime}\left(G-Y_{1}\right) \geq t+4-t=4$. By Theorem 2.2.2, $\tau(G-Y)=\tau\left(\left(G-Y_{1}\right)-Y_{2}\right) \geq 2$.

For any $e_{1}, e_{2} \in E(G)$, since $G$ has no essential (4+t)-edge-cut, $G-Y$ has no essential 2-edge-cut. Therefore, $\left\{e_{1}, e_{2}\right\}$ is not an essential edge-cut of $G-Y$. By Theorem 1.1.2, $G-Y$ has a spanning $\left(e_{1}, e_{2}\right)$-trail.

Let $t=0$ in Theorem 2.1.4, we obtain a result stronger than Theorem 2.1.2.

Corollary 2.2.3 Let $G$ be a graph with $\kappa^{\prime}(G) \geq 4$. Then $L(G)$ is 2-hamiltonian-connected if and only if $\kappa(L(G)) \geq 5$.

Lemma 2.2.4 If $\tau\left(G_{o}\right) \geq 2$ and $\kappa(L(G)) \geq 3$, then $\forall e_{1}, e_{2} \in E(G), G$ has a dominating $\left(e_{1}, e_{2}\right)$-trail. Therefore, $L(G)$ is hamiltonian-connected.

Proof. Let $e_{1}, e_{2} \in E(G)$ be given. Note that a spanning $\left(e_{1}, e_{2}\right)$-trail of $G_{o}$ yields a dominating $\left(e_{1}, e_{2}\right)$-trail of $G$. For $i=1,2$, let $e_{i}=u_{i} v_{i}$, and suppose $d\left(u_{1}\right) \leq d\left(u_{2}\right), d\left(u_{i}\right) \leq$ $d\left(v_{i}\right)$. Since $G$ does not have an essential 2-edge-cut, for each $i=1,2, d_{G}\left(v_{i}\right) \geq 3$ and so $v_{i} \in V\left(G_{o}\right)$.

We shall show that in each of the possible cases, $G$ has a dominating $\left(e_{1}, e_{2}\right)$-trail.

Case 1: $\quad e_{1}, e_{2} \notin E\left(G_{o}\right)$. 
By Theorem 1.2.2(i), $G_{o}$ is collapsible since $\tau\left(G_{o}\right) \geq 2$. Let $v_{1}=x, v_{2}=y$. By Theorem 1.2.1, there exists $(x, y)$-trail $T$ of $G_{o}$ such that $V(T)=V\left(G_{o}\right)$. Therefore, $\left\{e_{1}\right\} \bigcup E(T) \bigcup\left\{e_{2}\right\}$ is a dominating $\left(e_{1}, e_{2}\right)$-trail of $G$.

Case 2: $\quad e_{1} \notin E\left(G_{o}\right), e_{2} \in E\left(G_{o}\right)$.

Then subdividing $e_{2}$ by inserting a new vertex $y$, we get a new graph $G_{o}\left(e_{2}\right)$. Since $\tau\left(G_{o}\right) \geq 2, F\left(G_{o}\left(e_{2}\right)\right) \leq 1$.

By Theorem 1.2.5, $G_{o}\left(e_{2}\right)$ is collapsible since $\kappa^{\prime}\left(G_{o}\left(e_{2}\right)\right) \geq 2$. By the notation $e_{1}=$ $u_{1} v_{1}$ with $d\left(u_{1}\right) \leq d\left(v_{1}\right)$, we must have $v_{1} \in V\left(G_{o}\right)$. Let $v_{1}=x$. By Theorem 1.2.1, there exists a spanning $(x, y)$-trail $T$ of $G_{o}\left(e_{2}\right)$. Since $T$ is an $(x, y)$-trail, exact one of $u_{2} y$ and $y v_{2}$ is in $T$. Assume $u_{2} y \in E(T)$. Then $\left\{e_{1}\right\} \bigcup\left(E(T)-\left\{u_{2} y\right\}\right) \bigcup\left\{e_{2}\right\}$ is a dominating $\left(e_{1}, e_{2}\right)$-trail of $G$.

Case 3: $e_{1}, e_{2} \in E\left(G_{o}\right)$.

Then subdividing $e_{1}, e_{2}$ by inserting new vertices $x$ and $y$ in $e_{1}$ and $e_{2}$ respectively, we get a new graph $G_{o}\left(e_{1}, e_{2}\right)$. Since $\tau\left(G_{o}\right) \geq 2, F\left(G_{o}\left(e_{1}, e_{2}\right)\right) \leq 2$.

By Theorem 1.2.6, $G_{o}\left(e_{1}, e_{2}\right)$ is either collapsible or its reduction is a $K_{2, t}$.

If $G_{o}\left(e_{1}, e_{2}\right)$ is collapsible, then there exists a spanning $(x, y)$-trail $T$ of $G_{o}\left(e_{1}, e_{2}\right)$. Without loss of generality, assume $u_{1} x, u_{2} y \in E(T)$. Then $\left\{e_{1}\right\} \bigcup\left(E(T)-\left\{u_{1} x, u_{2} y\right\}\right) \bigcup\left\{e_{2}\right\}$ is a dominating $\left(e_{1}, e_{2}\right)$-trail of $G$.

If the reduction of $G_{o}\left(e_{1}, e_{2}\right)$ is isomorphic to a $K_{2, t}$, then denote $V\left(K_{2, t}\right)=\left\{x_{1}, x_{2}\right\} \bigcup\left\{y_{1}, \cdots, y_{t}\right\}$, where $x_{1}, x_{2}$ are the two nonadjacent vertices of degree $t$ and where $\left\{y_{1}, \cdots, y_{t}\right\}$ are the vertices of degree 2 other than $\left\{x_{1}, x_{2}\right\}$. Since $G_{o}$ is collapsible and $\kappa^{\prime}\left(G_{o}\right) \geq 3$, then $t=2$ and $\left\{y_{1}, y_{2}\right\}=\{x, y\}$. Therefore, $\left\{e_{1}, e_{2}\right\}$ is an essential 2-edge-cut, a contradiction.

Lemma 2.2.5 If $\kappa(L(G)) \geq 7$, then $\tau(G-Y)_{o} \geq 2$ for any $Y \subset V(L(G))=E(G)$ with $|Y| \leq 2$.

Proof. Note that $\kappa^{\prime}\left(G_{o}\right) \geq 3$ and $\kappa\left(L\left(G_{o}\right)\right) \geq 7$. We mainly use Theorem 2.2.1 in each of the possible cases to prove $\tau(G-Y)_{o} \geq 2$. 
Case 1: $Y=\phi$.

By Theorem 2.2.1, $\tau\left(G_{o}-\{x\}\right) \geq 2$ for any $x \in E(G)$. So $\tau\left(G_{o}\right) \geq 2$.

Case 2: $Y=\{e\}$.

Let $e=u v$ and suppose $d(u) \leq d(v)$.

Subcase 2.1: $u \in D_{1}$.

Since $\kappa(L(G)) \geq 7, G$ does not have an essential 6-edge-cut. And $E=\left\{e^{\prime} \mid e^{\prime}\right.$ is incident with $v$ and $\left.e^{\prime} \neq e\right\}$ is an essential edge-cut. So $|E| \geq 7$. Thus $d(v) \geq 7+1=8$. Therefore, $(G-e)_{o}=G_{o}$. By Theorem 2.2.1, $\tau(G-e)_{o}=\tau\left(G_{o}\right) \geq 2$.

Subcase 2.2: $u \in D_{2}$.

Suppose $e^{\prime}=u v^{\prime}$ with $v^{\prime} \neq v$. Since $E=\left\{e^{\prime \prime} \mid e^{\prime \prime}\right.$ is incident with $v$ and $\left.e^{\prime \prime} \neq e\right\} \cup\left\{e^{\prime}\right\}$ is an essential edge-cut and $\kappa(L(G)) \geq 7$, then $|E| \geq 7$. So $d(v)=\left|E \backslash\left\{e^{\prime}\right\} \cup\{e\}\right| \geq 7$. Similarly, $d\left(v^{\prime}\right) \geq 7$. Contract $e^{\prime}$ such that $e \in G_{o}$ when we obtain $G_{o}$ from $G$, then $(G-e)_{o}=G_{o}-e$. By Theorem 2.2.1, $\tau(G-e)_{o}=\tau\left(G_{o}-e\right) \geq 2$.

Subcase 2.3: $u \in D_{3}$.

Then $d(v) \geq 6$. Let $e^{\prime}=u v^{\prime}, e^{\prime \prime}=u v^{\prime \prime}$ with $e^{\prime} \neq e^{\prime \prime}$ and $v^{\prime}, v^{\prime \prime} \neq v$. Note that by Theorem 2.2.1, $G_{o}-e$ has two edge disjoint spanning trees $T^{\prime}$ and $T^{\prime \prime}$. Since $d_{G_{o}-e}(u)=2$, exactly one of $e^{\prime}$ and $e^{\prime \prime}$ is in $T^{\prime}$ and the other is in $T^{\prime \prime}$. Assume $e^{\prime} \in T^{\prime}$ and $e^{\prime \prime} \in T^{\prime \prime}$. Then $T^{\prime}-e^{\prime}$ and $T^{\prime \prime}-e^{\prime \prime}$ are two edge disjoint spanning trees of $\left(G_{o}-e\right) / e^{\prime}$. And $(G-e)_{o}=\left(G_{o}-e\right) / e^{\prime}$. Thus $\tau(G-e)_{o}=\tau\left(\left(G_{o}-e\right) / e^{\prime}\right) \geq 2$.

Subcase 2.4: $\quad d(u) \geq 4$.

Since $d_{G-e}(u) \geq 3,(G-e)_{o}=G_{o}-e$. By Theorem 2.2.1, $\tau(G-e)_{o}=\tau\left(G_{o}-e\right) \geq 2$.

Case 3: $Y=\left\{e, e^{\prime}\right\}$.

Let $e=u_{1} v_{1}, e^{\prime}=u_{2} v_{2}$ and suppose $d\left(u_{1}\right) \leq d\left(u_{2}\right), d\left(u_{i}\right) \leq d\left(v_{i}\right)$, for each $i=1,2$.

Subcase 3.1: $u_{1}, u_{2} \in D_{1}$. 
Then $d\left(v_{1}\right), d\left(v_{2}\right) \geq 8$. So $\left(G-\left\{e, e^{\prime}\right\}\right)_{o}=G_{o}$. By Theorem 2.2.1, $\tau\left(G-\left\{e, e^{\prime}\right\}\right)_{o}=$ $\tau\left(G_{o}\right) \geq 2$.

Subcase 3.2: $u_{1} \in D_{1}, u_{2} \notin D_{1}$.

Then $\left(G-\left\{e, e^{\prime}\right\}\right)_{o}=\left((G-e)-e^{\prime}\right)_{o}$. Apply the same argument as in Subcases 2.2, 2.3 and 2.4 to $G-e$. We conclude that $\tau\left(G-\left\{e, e^{\prime}\right\}\right)_{o} \geq 2$.

Subcase 3.3: $u_{1}, u_{2} \notin D_{1}$.

Subcase 3.3.1: $u_{1}, u_{2} \in D_{2}$.

Then $d\left(v_{i}\right) \geq 7$, for each $i=1,2$. If $u_{1}=u_{2}$, contract $e^{\prime}$ such that $e \in G_{o}$ when we obtain $G_{o}$ from $G$. Then $\left(G-\left\{e, e^{\prime}\right\}\right)_{o}=G_{o}-e$. Thus $\tau\left(G-\left\{e, e^{\prime}\right\}\right)_{o}=\tau\left(G_{o}-e\right) \geq$ 2. If $u_{1} \neq u_{2}$, we obtain $G_{o}$ by contracting other edges such that $e, e^{\prime} \in G_{o}$. Then $\left(G-\left\{e, e^{\prime}\right\}\right)_{o}=G_{o}-\left\{e, e^{\prime}\right\}$. By Theorem 2.2.1, $\tau\left(G-\left\{e, e^{\prime}\right\}\right)_{o}=\tau\left(G_{o}-\left\{e, e^{\prime}\right\}\right) \geq 2$.

Subcase 3.3.2: $\quad u_{1} \in D_{2}, d\left(u_{2}\right) \geq 3$.

We obtain $G_{o}$ by contracting the other edge such that $e \in G_{o}$. If $d\left(u_{2}\right)=3,(G-$ $\left.\left\{e, e^{\prime}\right\}\right)_{o}=\left(G_{o}-\left\{e, e^{\prime}\right\}\right) / e^{\prime \prime}$ where $e^{\prime \prime}=u_{2} v^{\prime \prime}$ with $v^{\prime \prime} \neq v_{2}$. Similar to Subcases 2.2 and 2.3, $\tau\left(G-\left\{e, e^{\prime}\right\}\right)_{o} \geq 2$. If $d\left(u_{2}\right) \geq 4$, then $\left(G-\left\{e, e^{\prime}\right\}\right)_{o}=G_{o}-\left\{e, e^{\prime}\right\}$. Thus $\tau\left(G-\left\{e, e^{\prime}\right\}\right)_{o}=\tau\left(G_{o}-\left\{e, e^{\prime}\right\}\right) \geq 2$.

Subcase 3.3.3: $d\left(u_{1}\right), d\left(u_{2}\right) \geq 3$.

If $u_{1}, u_{2} \in D_{3}$ and $u_{1}=u_{2}$, then suppose $e^{\prime \prime}=u_{1} v^{\prime \prime}$ with $v^{\prime \prime} \neq v_{1}, v_{2}$. Therefore, $\left(G-\left\{e, e^{\prime}\right\}\right)_{o}=\left(G_{o}-\left\{e, e^{\prime}\right\}\right) / e^{\prime \prime}$. Since $\tau\left(G_{o}-e\right) \geq 2$ and $d_{G_{o}-e}\left(u_{1}\right)=2, G_{o}-e$ has two edge disjoint spanning trees $T^{\prime}$ and $T^{\prime \prime}$ which contain $e^{\prime}$ and $e^{\prime \prime}$ respectively. Therefore, $T^{\prime}-e^{\prime}$ and $T^{\prime \prime}-e^{\prime \prime}$ are two edge disjoint spanning trees of $\left(G_{o}-\left\{e, e^{\prime}\right\}\right) / e^{\prime \prime}$. So $\tau\left(G-\left\{e, e^{\prime}\right\}\right)_{o}=\tau\left(G_{o}-\left\{e, e^{\prime}\right\}\right) / e^{\prime \prime} \geq 2$. If $u_{1}, u_{2} \in D_{3}$ and $u_{1} \neq u_{2}$, then suppose $e_{3}=u_{1} v_{3}, e_{4}=u_{2} v_{4}$ with $v_{3} \neq v_{1}, v_{4} \neq v_{2}$. Then $\left(G-\left\{e, e^{\prime}\right\}\right)_{o}=\left(G_{o}-\left\{e, e^{\prime}\right\}\right) /\left\{e_{3}, e_{4}\right\}$. Similar to Subcase 2.3, $\tau\left(G-\left\{e, e^{\prime}\right\}\right)_{o}=\tau\left(\left(G_{o}-\left\{e, e^{\prime}\right\}\right) /\left\{e_{3}, e_{4}\right\}\right) \geq 2$.

If $u_{1} \in D_{3}$ and $d\left(u_{2}\right) \geq 4$, let $e_{3}=u_{1} v_{3}$ with $v_{3} \neq v_{1}$. Then $\left(G-\left\{e, e^{\prime}\right\}\right)_{o}=$ $\left(G_{o}-\left\{e, e^{\prime}\right\}\right) / e_{3}$. Similar to Subcases 2.3 and 2.4, $\tau\left(G-\left\{e, e^{\prime}\right\}\right)_{o} \geq 2$. 
If $d\left(u_{1}\right), d\left(u_{2}\right)=4$ and $u_{1}=u_{2}$, suppose $e_{3}=u_{1} v_{3}$ with $v_{3} \neq v_{1}, v_{2}$. Then $(G-$ $\left.\left\{e, e^{\prime}\right\}\right)_{o}=\left(G_{o}-\left\{e, e^{\prime}\right\}\right) / e_{3}$. Since $\tau\left(G_{o}-\left\{e, e^{\prime}\right\}\right) \geq 2$, similar to subcase $2.3 \tau\left(G_{o}-\right.$ $\left.\left\{e, e^{\prime}\right\}\right) / e_{3} \geq 2$. If $d\left(u_{1}\right), d\left(u_{2}\right)=4$ and $u_{1} \neq u_{2}$ or $d\left(u_{2}\right)>4$, then $\left(G-\left\{e, e^{\prime}\right\}\right)_{o}=$ $G_{o}-\left\{e, e^{\prime}\right\}$. Thus $\tau\left(G-\left\{e, e^{\prime}\right\}\right)_{o} \geq 2$.

\section{Proof of Theorem 2.1.5}

Let $Y \subset V(L(G))=E(G)$ with $|Y| \leq s$. If $|Y| \leq 2$, let $Y_{1}=Y$ and if $|Y| \geq 3$, let $Y_{1} \subset Y$ with $\left|Y_{1}\right|=2$ and $Y_{2}=Y-Y_{1},\left|Y_{2}\right| \leq s-2$. Since $\kappa(L(G)) \geq s+5 \geq 7$, $\kappa\left(L(G)-Y_{2}\right) \geq 7$. By Lemma 2.2.5, we have $\tau(G-Y)_{o}=\tau\left(\left(G-Y_{2}\right)-Y_{1}\right)_{o} \geq 2$. By Lemma 2.2.4, $L(G)-Y$ is hamiltonian-connected. Thus $L(G)$ is s-hamiltonian-connected.

When $s=2$, the corollary below extends Theorem 2.1.3.

Corollary 2.2.6 Every 7-connected line graph is 2-hamiltonian-connected.

\section{$2.3 \quad$ A Remark}

We conclude this chapter with the following remark.

Theorem 2.1.5 suggests that for any $s \geq 2$, there exists a minimum number $f(s)$ such that if $\kappa(L(G)) \geq f(s)$, then $L(G)$ is $s$-hamiltonian-connected. What is the exact value of $f(s)$ ? Theorem 2.1.5 showed that for $s \geq 2, f(s) \leq s+5$. As any $s$-hamiltonian-connected graph must be $(s+3)$-connected, we conjecture that for large values of $s, f(s)=s+3$. 


\section{Chapter 3}

\section{Characterization of Supereulerian Graphs in $C_{2}(6, k)$}

\subsection{Introduction}

For integers $h, l$ and $k$ with $l>0,0<h \leq 3$ and $k \geq 0$, let $C_{h}(l, k)$ denote the family of $h$-edge-connected graphs $G$ such that for every bond $X$ with two or three edges, each component of $G-X$ has at least $(|V(G)|-k) / l$ vertices.

The supereulerian problem of a graph $G$ is to determine whether $G$ is a supereulerian graph. This problem was first raised by Boesch et al [12]. They pointed out in [12] that this problem is very difficult. Pulleyblank [26] showed that determining if a graph is supereulerian is NP-complete. For literatures of the problem, see Catlin's survey [14] and its supplement [18]. Catlin and $\mathrm{Li}$ [16] are the first pioneers who consider the problem of characterizing supereulerian graphs in the family $C_{h}(l, k)$. Their study was followed by several researchers.

Definition 3.1.1 Let $K_{2,3}(e)$ denote the graph obtained from $K_{2,3}$ by replacing an edge $e \in E\left(K_{2,3}\right)$ by a path of length 2. Let $m, l, t$ be natural numbers with $t \geq 2$ and $m, l \geq 1$. Let $K_{2, t}\left(u, u^{\prime}\right)$ be $K_{2, t}$ with $u, u^{\prime}$ being the nonadjacent vertices of degree $t$. Let 
$K_{2, t}^{\prime}\left(u, u^{\prime}, u^{\prime \prime}\right)$ be the graph obtained from $K_{2, t}\left(u, u^{\prime}\right)$ by adding a new vertex $u^{\prime \prime}$ that joins to $u^{\prime}$ only. Hence, $u^{\prime \prime}$ has degree 1 and $u$ has degree $t$ in $K_{2, t}^{\prime}\left(u, u^{\prime}, u^{\prime \prime}\right)$. Let $K_{2, t}^{\prime \prime}\left(u, u^{\prime}, u^{\prime \prime}\right)$ be the graph obtained from $K_{2, t}\left(u, u^{\prime}\right)$ by adding a new vertex $u^{\prime \prime}$ that joins to a vertex of degree 2 of $K_{2, t}$. Hence, $u^{\prime \prime}$ has degree 1 and both $u$ and $u^{\prime}$ have degree $t$ in $K_{2, t}^{\prime \prime}\left(u, u^{\prime}, u^{\prime \prime}\right)$. Let $S(m, l)$ be the graph obtained from $K_{2, m}\left(u, u^{\prime}\right)$ and $K_{2, l}^{\prime}\left(w, w^{\prime}, w^{\prime \prime}\right)$ by identifying $u$ with $w$, and $w^{\prime \prime}$ with $u^{\prime}$; let $J(m, l)$ denote the graph obtained from $K_{2, m+1}$ and a $K_{2, l}^{\prime}\left(w, w^{\prime}, w^{\prime \prime}\right)$ by identifying $w$ with 2-vertex and $w^{\prime \prime}$ with an $(m+1)$-vertex in $K_{2, m+1}$, respectively.

Let $\mathcal{F}^{\prime}=\left\{S(1,2), S(2,3), S(1,4), J(2,2), K_{2,3}, K_{2,5}\right\}$ (see Figure 3.1).

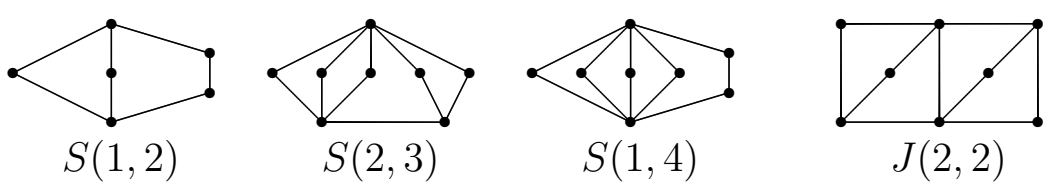

Figure 3.1. The graphs in $\mathcal{F}^{\prime}$.

We summerize the former results in the area.

Theorem 3.1.2 (Catlin and $\mathrm{Li}$, Theorem 6 of [16]) If $G \in C_{2}(5,0)$, then $G \in \mathcal{S}$ if and only if $G$ can not be contracted to $K_{2,3}$.

Theorem 3.1.3 (Broersma and Xiong, Theorem 7 of [13]) Suppose that $G \in C_{2}(5,2)$ and $n \geq 13$. Then $G \in \mathcal{S}$ if and only if $G$ can not be contracted to $K_{2,3}$ or to $K_{2,5}$.

Theorem 3.1.4 (Li, Lai and Zhan, Theorem 1.3 of [8]) Suppose that $G \in C_{2}(6,0)$. Then $G \in \mathcal{S}$ if and only if $G$ can not be contracted to a member in $\left\{K_{2,3}, K_{2,5}\right.$ or $\left.K_{2,3}(e)\right\}$.

Theorem 3.1.5 (X. Li, D. Li and H.-J. Lai, Theorem 14 of [24]) Let $G \in C_{2}(6,5)$ be a graph with $n=|V(G)|>35$. Then $G \in \mathcal{S}$ if and only if $G$ can not be contracted to a member in $\mathcal{F}^{\prime}$. 
Chen [18] and Xiong et al [27] also studied the supereulerian problem for graphs in $C_{3}(l, k)$. Jeager [21] and Catlin [2] proved independently that every 4-edge-connected graph is supereulerian, and so the study is of interest only when $h<4$.

The supereulerian problem for graphs in $C_{2}(6, k)$, for an arbitrary positive integer $k$, remains open [24]. The main purpose of this paper is to answer this question. The attempt to answer this question leads us to prove an associate result which is of interests in its own. We prove the following.

Theorem 3.1.6 Let $k>0$ be an integer. Then there exists an integer $N(k) \leq 7 k$ such that, for any graph $G \in C_{2}(6, k)$ with $|V(G)|>N(k), G \in \mathcal{S}$ if and only if $G$ can not be contracted to a member in $\mathcal{F}^{\prime}$.

\subsection{An Associate Result}

The main purpose of this section is to prove the following associate result, which plays a key role in the proof of Theorem 3.1.6.

Theorem 3.2.1 If $G$ is a 2-edge-connected reduced graph which satisfies

(i) $d_{2}+d_{3} \leq 6$,

(ii) $d_{3}+d_{5} \leq 2$,

then either $G \in \mathcal{S}$ or $G \in \mathcal{F}^{\prime}$.

Definition 3.2.2 Let $\mathcal{A}=\{G: G$ is a 2-edge-connected reduced graph which satisfies $d_{2}+d_{3} \leq 6$ and $\left.d_{3}+d_{5} \leq 2\right\}$ and $\mathcal{A}_{3}=\{G \in \mathcal{A}: G \notin \mathcal{S}$ and $F(G)=3\}$. Then by the following Lemma 3.2.3, for any $G \in \mathcal{A}_{3}$, we have $d_{2}+d_{3}=6, d_{3}+d_{5}=2$ and $d_{j}=0$ for all $j \geq 6$.

We first prove some needed lemmas. 
Lemma 3.2.3 If $G \in \mathcal{A}$, then either $G$ is Eulerian or $F(G) \leq 3$. Furthermore, if $F(G)=3$, then either $G$ is Eulerian or $d_{2}+d_{3}=6, d_{3}+d_{5}=2$ and $d_{j}=0$ for all $j \geq 6$.

Proof. Note that $F(G) \leq 4$ since

$$
\begin{aligned}
2 F(G) & =4|V(G)|-2|E(G)|-4=4 \sum_{i \geq 2} d_{i}-\sum_{i \geq 2} i d_{i}-4 \\
& =2\left(d_{2}+d_{3}\right)-\left(d_{3}+d_{5}\right)-\sum_{i \geq 6}(i-4) d_{i}-4 \\
& \leq 8-\left(d_{3}+d_{5}\right)-\sum_{i \geq 6}(i-4) d_{i} \leq 8 .
\end{aligned}
$$

If $F(G)=4$, then $d_{3}+d_{5}=0$ and $d_{j}=0$ for all $j \geq 6$. Since $G$ has no odd-degree vertices, $G$ is Eulerian.

Suppose $F(G)=3$. If there exists some $j \geq 6$ such that $d_{j}>0$, then $j=6, d_{6}=1$ and $d_{3}+d_{5}=0$. Therefore, $G$ is Eulerian. If $d_{j}=0$ for all $j \geq 6$, then $d_{2}+d_{3}=6$, $d_{3}+d_{5}=2$.

Lemma 3.2.4 If $G \in \mathcal{A}_{3}$, then we must have $\left(d_{2}, d_{3}, d_{5}\right) \in\{(4,2,0),(5,1,1),(6,0,2)\}$.

Proof. If $d_{3}=2$, then $d_{2}=4$ and $d_{5}=0$. If $d_{3}=1$, then $d_{2}=5$ and $d_{5}=1$. If $d_{3}=0$, then $d_{2}=6$ and $d_{5}=2$.

Lemma 3.2.5 If a 2-edge-connected graph $G \notin \mathcal{S}$ and $|O(G)|=2$, then $O(G)$ is an independent set.

Proof. $G$ has two odd vertices, say $u$ and $v$. If $u$ and $v$ are adjacent, then $G-u v$ is Eulerian. Therefore, $G \in \mathcal{S}$, a contradiction.

Lemma 3.2.6 If $G$ is reduced and $e=u v$ where $u, v \in D_{2}(G)$, then the following statements hold.

(i) If $G / e \in \mathcal{S}$, then $G \in \mathcal{S}$.

(ii) $F(G / e)=F(G)-1$. 
Proof. Part (i) follows from Lemma 3 of [2]. To prove Part (ii), we first show that the $a(G / e) \leq 2$.

By Corollary 1.2.4, $\frac{|E(H)|}{|V(H)|-1}<2$, for any nontrivial induced subgraph $H$ of $G$. We now argue by contradiction to show that $a(G / e) \leq 2$, and assume that $G / e$ has a nontrivial induced subgraph $L^{\prime}$ with $\frac{\left|E\left(L^{\prime}\right)\right|}{\left|V\left(L^{\prime}\right)\right|-1}>2$. Let $L$ be the induced subgraph of $G$ such that either $L=L^{\prime}$, or $e \in E(L)$ and $L / e=L^{\prime}$. Since $\frac{|E(H)|}{|V(H)|-1}<2$, for any nontrivial induced subgraph $H$ of $G$, we must have $e \in E(L)$.

Since $e \in E(L)$, both $|E(L)|=\left|E\left(L^{\prime}\right)\right|+1$ and $|V(L)| \leq\left|V\left(L^{\prime}\right)\right|+1$ hold. Since $\frac{\left|E\left(L^{\prime}\right)\right|}{\left|V\left(L^{\prime}\right)\right|-1}>2,\left|E\left(L^{\prime}\right)\right| \geq 2\left|V\left(L^{\prime}\right)\right|-1$, which implies that

$$
\frac{|E(L)|}{|V(L)|-1} \geq \frac{\left|E\left(L^{\prime}\right)\right|+1}{\left|V\left(L^{\prime}\right)\right|} \geq \frac{2\left|V\left(L^{\prime}\right)\right|}{\left|V\left(L^{\prime}\right)\right|}=2,
$$

contrary to $\frac{|E(L)|}{|V(L)|-1}<2$.

Thus $a(G / e) \leq 2$. By Theorem 1.2.2(v),

$$
\begin{aligned}
2 F(G / e) & =4|V(G / e)|-2|E(G / e)|-4=4(|V(G)|-1)-2(|E(G)|-1)-4 \\
& =4|V(G)|-2|E(G)|-4-2=2 F(G)-2,
\end{aligned}
$$

and so Part (ii) holds.

Notation 3.2.7 Suppose that $H$ is a subgraph of a graph L. Let $d_{i, L}(H)$ denote the number of vertices of $H$ of degree $i$ in $L$, and $v_{H}$ the vertex in $L / H$ onto which $H$ is contracted.

Lemma 3.2.8 Let $H$ be a subgraph of a graph L. Then each of the following statement holds:

(i) $4|V(H)|-2|E(H)|-4=\sum_{i>0}(4-i) d_{i, L}(H)+d\left(v_{H}\right)-4$. In particular, if $d_{i, L}(v)=0$ for all $i \geq 6, i=1$ and $H$ is reduced, then $2 F(H)=2 d_{2, L}(H)+d_{3, L}(H)-d_{5, L}(H)+d\left(v_{H}\right)-4$. (ii) For any $H, F(H-e) \leq F(H)+1$.

Proof. First notice that

$$
2|E(H)|=\sum_{v \in H} d_{L}(v)-d\left(v_{H}\right)=\sum_{i>0} i d_{i, L}(H)-d\left(v_{H}\right) .
$$


Therefore,

$$
\begin{aligned}
4|V(H)|-2|E(H)|-4 & =4 \sum_{i>0} d_{i, L}(H)-\left(\sum_{i>0} i d_{i, L}(H)-d\left(v_{H}\right)\right)-4 \\
& =\sum_{i>0}(4-i) d_{i, L}(H)+d\left(v_{H}\right)-4 .
\end{aligned}
$$

So part (i) holds.

For any $H$, suppose $X$ is a set of edges not in $H$, but adding $X$ to $H$ will result in a graph with 2 edge disjoint spanning trees. Then adding $X \bigcup e$ to $H-e$ will also result in a graph with 2 edge-disjoint spanning trees. Therefore, part (ii) holds.

Lemma 3.2.9 If $G \in \mathcal{A}_{3}$, then either $G \in\{S(1,2), S(1,4)\}$ or $D_{2}(G)$ is an independent set.

Proof. Suppose there exist $u, v \in D_{2}(G)$ such that $e=u v \in E(G)$.

Let $G^{\prime}=G /$ e. By Lemma 3.2.6 (i), $G^{\prime} \notin \mathcal{S}$. By Lemma 3.2.3 and Lemma 3.2.6 (ii), $F\left(G^{\prime}\right) \leq F(G)-1 \leq 3-1=2$. Since $\kappa^{\prime}\left(G^{\prime}\right) \geq 2$, the reduction of $G^{\prime}$ is not $K_{2}$ or $K_{2,1}$. Since $G^{\prime} \notin \mathcal{S}, G^{\prime}$ is not collapsible. Let $G_{0}$ denote the reduction of $G^{\prime}$. By Theorem 1.2.2 (ii) and Theorem 1.2.6,

$$
G_{0}=K_{2, t} \text {, for some } t \geq 3 \text {, where } t \text { is odd. }
$$

Let $v_{e}$ denote the new vertex obtained from contracting the edge $e$ of $G$. Then $G^{\prime}$ has at most one nontrivial collapsible subgraph, as any nontrivial collapsible subgraph must contain $v_{e}$. Since $d_{2}(G)+d_{3}(G)=6, d_{3}(G)+d_{5}(G)=2$ and $d_{j}(G)=0$ for all $j \geq 6$, we have $t=3$ or 5 , and so $G_{0} \in\left\{K_{2,3}, K_{2,5}\right\}$ by (3.2). Let $H^{\prime}$ denote the collapsible subgraph of $G^{\prime}$ containing $v_{e}$, and $H$ denote the preimage of $H^{\prime}$ from contraction .

Suppose $H=K_{2}$. Then $H^{\prime}$ contains only one vertex $v_{e}$. Therefore, $H=\{e\}$ and $G / e=G^{\prime}$. If $G / e=K_{2,3}$, then $G=S(1,2)$. If $G / e=K_{2,5}$, then $G=S(1,4)$.

Next we will show that $H=K_{2}$. By contradiction, suppose that $H \neq K_{2}$. Then $H^{\prime}$ is a nontrivial collapsible subgraph of $G^{\prime}$. Therefore, $\kappa^{\prime}\left(H^{\prime}\right) \geq 2$. So $\kappa^{\prime}(H) \geq 2$. By 
Theorem 1.2.5, since $H$ is not a collapsible subgraph of $G, F(H)>1$. Then $G / H=$ $G^{\prime} / H^{\prime}=G_{0} \in\left\{K_{2,3}, K_{2,5}\right\}$.

Suppose $G_{0}=K_{2,3}$. Since $u, v \in H, d_{2, G}(H) \geq 2$. Note that $d_{2}(G)+d_{3}(G)=6$ and $d_{3}(G)+d_{5}(G)=2$. So there are two possibilities (see Table 3.1). Computing $F(H)$ by using Lemma 3.2.8(i), we have $F(H)=1$, contrary to $F(H)>1$.

\begin{tabular}{|l|l|l|l|l|}
\hline$d\left(v_{H}\right)$ & $d_{2, G}(H)$ & $d_{3, G}(H)$ & $d_{5, G}(H)$ & $F(H)$ \\
\hline 2 & 2 & 0 & 0 & 1 \\
\hline 3 & 2 & 0 & 1 & 1 \\
\hline
\end{tabular}

Table 3.1. The table for computing $F(H)$ when $G_{0}=K_{2,3}$.

Suppose $G_{0}=K_{2,5}$. Note that $d_{2, G}(H) \geq 2, d_{2}(G)+d_{3}(G)=6$ and $d_{3}(G)+d_{5}(G)=2$. Then there is only one possibility (see Table 3.2 ). Computing $F(H)$ by using Lemma 3.2.8(i), $F(H)=1$, contrary to $F(H)>1$.

\begin{tabular}{|l|l|l|l|l|}
\hline$d\left(v_{H}\right)$ & $d_{2, G}(H)$ & $d_{3, G}(H)$ & $d_{5, G}(H)$ & $F(H)$ \\
\hline 2 & 2 & 0 & 0 & 1 \\
\hline
\end{tabular}

Table 3.2. The table for computing $F(H)$ when $G_{0}=K_{2,5}$.

Thus, if $G \notin \mathcal{S}$, then either $G \in\{S(1,2), S(1,4)\}$ or $D_{2}(G)$ is an independent set.

Lemma 3.2.10 If $K$ is an induced subgraph of a graph $L$, then each of the following holds:

(i) If $d_{3}(L)+d_{5}(L) \leq 2, d_{2}(L)+d_{3}(L) \leq 6$ and $L / K \in \mathcal{F}^{\prime}$, then $2|V(K)|-|E(K)|-2 \leq 1$.

(ii) If $L \in \mathcal{A}$ and $L / K \in \mathcal{F}^{\prime}$, then we have $F(K) \leq 1$. Moreover, $F(K)=1$ only if $L / K \in\left\{K_{2,3}, K_{2,5}\right\}$ and $d_{2}(L)+d_{3}(L)=6$.

Proof. First we prove part (i). Since $L / K \in \mathcal{F}^{\prime}$, we have $d_{3}(L)+d_{5}(L)=2$. If $d_{2}(L)+d_{3}(L)=6$, then we have the following possibilities (see Table 3.3. The last column of Table 3.3 defines the Type of the subgraphs arising from contraction, which will be used in the proof of Lemma 3.2.13). 


\begin{tabular}{|l|l|l|l|l|l|l|}
\hline$L / K$ & $d\left(v_{K}\right)$ & $d_{2, L}(K)$ & $d_{3, L}(K)$ & $d_{5, L}(K)$ & $2|V(K)|-|E(K)|-2 \leq$ & Type \\
\hline$K_{2,3}$ & 2 & 2 & 0 & 0 & 1 & $\mathrm{~A}$ \\
\hline & 3 & 1 & 1 & 0 & 1 & $\mathrm{~B}$ \\
\hline & & 2 & 0 & 1 & 1 & $\mathrm{C}$ \\
\hline$K_{2,5}$ & 2 & 2 & 0 & 0 & 1 & $\mathrm{D}$ \\
\hline & 5 & 0 & 1 & 0 & 1 & $\mathrm{E}$ \\
\hline & & 1 & 0 & 1 & 1 & $\mathrm{~F}$ \\
\hline$S(1,2)$ & 2 & 1 & 0 & 0 & 0 & $\mathrm{G}$ \\
\hline & 3 & 0 & 1 & 0 & 0 & $\mathrm{H}$ \\
\hline & & 1 & 0 & 1 & 0 & $\mathrm{I}$ \\
\hline$S(1,4)$ & 2 & 1 & 0 & 0 & 0 & $\mathrm{~J}$ \\
\hline & 5 & 0 & 0 & 1 & 0 & $\mathrm{~K}$ \\
\hline$S(2,3)$ & 2 & 1 & 0 & 0 & 0 & $\mathrm{~L}$ \\
\hline & 3 & 0 & 1 & 0 & 0 & $\mathrm{M}$ \\
\hline & & 1 & 0 & 1 & 0 & $\mathrm{~N}$ \\
\hline & 4 & 0 & 0 & 0 & 0 & $\mathrm{O}$ \\
\hline & 5 & 0 & 0 & 1 & 0 & $\mathrm{P}$ \\
\hline$J(2,2)$ & 2 & 1 & 0 & 0 & 0 & $\mathrm{Q}$ \\
\hline & 3 & 0 & 1 & 0 & 0 & $\mathrm{R}$ \\
\hline & & 1 & 0 & 1 & 0 & $\mathrm{~S}$ \\
\hline & 4 & 0 & 0 & 0 & 0 & $\mathrm{~T}$ \\
\hline
\end{tabular}

Table 3.3. The table in the proof of Lemma 3.2.10

If $d_{2}(L)+d_{3}(L)<6$, then $d_{2, L}(K)$ decreases at least by one. Computing $2|V(K)|-$ $|E(K)|-2$ by using Lemma 3.2.8(i), $2|V(K)|-|E(K)|-2$ decreases at least by one. So $2|V(K)|-|E(K)|-2 \leq 0$. Hence part (i) holds.

If $L \in \mathcal{A}$, then $K$ is reduced. So $F(K)=2|V(K)|-|E(K)|-2 \leq 1$. From the proof of part (i), the equality holds only if $L / K \in\left\{K_{2,3}, K_{2,5}\right\}$ and $d_{2}(L)+d_{3}(L)=6$.

Definition 3.2.11 Let $u \in D_{2}(G)$ and $v \in D_{4}(G)$. Suppose $N(u)=\{v, w\}$ and $N(v)=$ $\{u, x, y, z\}$. Define $T(G)=(G-v)+\{y z, u x\}$ (see Figure 3.2). 


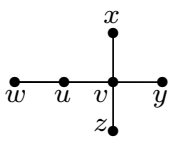

in $G$

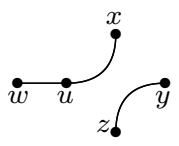

in $T(G)$

Figure 3.2. The operator $T$ on a graph $G$.

Lemma 3.2.12 Let $G$ be a 2-edge-connected reduced graph, and let $e=u v \in E(G)$ such that $u \in D_{2}(G)$ and $v \in D_{4}(G)$. Let $N(u)=\{v, w\}$ and $N(v)=\{u, x, y, z\}$. Then

(i) $T(G)$ is 2-edge-connected (relabelling the vertices if needed).

(ii) $a(T(G)) \leq 2$. Therefore, $F(K)=2|V(K)|-|E(K)|-2$ for any induced subgraph $K$ of $T(G)$.

(iii) If $T(G) \in \mathcal{S}$, then $G \in \mathcal{S}$.

(iv) $T(G)$ has at most two nontrivial collapsible subgraphs which must contain yz or ux.

(v) Any two vertices in $N(v)=\{u, x, y, z\}$ are not adjacent.

(vi) If $G \in \mathcal{A}$, then the reduction of $T(G)$ is also in $\mathcal{A}$.

Proof. Part (i) follows from Splitting Lemma (see [20], on page III. 29).

By contradiction, assume there exists an induced subgraph $K$ of $T(G)$ such that $|E(K)| /(|V(K)|-1)>2$, i.e. $|E(K)| \geq 2|V(K)|-1$. Suppose $H$ is the subgraph of $G$ corresponding to $K$. By Corollary 1.2.4, $|E(H)| /(|V(H)|-1)<2$. So $v \in H$. If both $u x$ and $y z$ are in $K$, then $|E(H)| /(|V(H)|-1)=(|E(K)|+2) /|V(K)| \geq(2|V(K)|+$ $1) /|V(K)|>2$, contrary to $|E(H)| /(|V(H)|-1)<2$. If exactly one of $u x$ and $y z$ is in $K$, then $|E(H)| /(|V(H)|-1)=(|E(K)|+1) /|V(K)| \geq 2|V(K)| /|V(K)|=2$, a contradiction. Thus $a(T(G)) \leq 2$ by (1.1). Hence, by Theorem 1.2.2(v), $F(K)=2|V(K)|-|E(K)|-2$, for any induced subgraph $K$ of $T(G)$. Part (ii) holds.

If $T(G) \in \mathcal{S}$, suppose $H$ is an spanning Eulerian subgraph of $T(G)$. Then $H$ must contain $u x$ since $d_{T(G)}(u)=2$. If $y z \notin H$, then $H-u x+u v+v x$ is an Eulerian subgraph of $G$. If $y z \in H$, then $H-u x-y z+u v+v x+v y+v z$ is an Eulerian subgraph of $G$. Thus $G \in \mathcal{S}$. Part (iii) holds.

Any collapsible subgraph of $T(G)$ must contain the edge $y z$ or $u x$. Otherwise, it is also a collapsible subgraph of $G$, contrary to that $G$ is reduced. So $T(G)$ has at most two 
nontrivial collapsible subgraphs. Part (iv) holds.

Note that $G$ is reduced, so there is no $C_{3}$ in $G$. It implies that part (v) holds.

Now we prove part (vi). Suppose $H^{\prime}$ is a maximum collapsible subgraph of $T(G)$. It suffices to prove that $T(G) / H^{\prime}$, denoted by $G_{1}$, still satisfies $d_{2}\left(G_{1}\right)+d_{3}\left(G_{1}\right) \leq 6$ and $d_{3}\left(G_{1}\right)+d_{5}\left(G_{1}\right) \leq 2$. First, note that the number of odd degree vertices will not increase by contracting a subgraph. Otherwise, if after the contraction, the number of odd degree vertices increases by 1 , then the number of odd vertices of the new graph obtained by contraction will be odd, contrary to that the number of odd vertices of a graph must be even. And since $G \in \mathcal{A}$, by Lemma 3.2.3, either $G$ has no odd vertices or $F(G) \leq 3$. If $F(G) \leq 2$, then either $G$ has no odd vertices or $G=K_{2, t}$ by Theorem 1.2.6. Since $d_{2}(G)+d_{3}(G) \leq 6, t \leq 6$. Hence the odd degree of $G$ is at most 5 . If $F(G)=3$, by Lemma 3.2.3, either $G$ has no odd vertices or $d_{j}=0$ for all $j \geq 6$. Thus if $G \in \mathcal{A}$, then the odd degree vertices of $G$ must be of degree 3 or 5 . After the contraction, we still have $d_{3}\left(G_{1}\right)+d_{5}\left(G_{1}\right) \leq 2$.

If $d_{2}\left(G_{1}\right)+d_{3}\left(G_{1}\right)>d_{2}(G)+d_{3}(G)$, then $d\left(v_{H^{\prime}}\right)=2$ or 3 . In each case, we will prove $H^{\prime}-y z$ is a collapsible subgraph of $G$, contrary to that $G$ is reduced.

Case 1: $d\left(v_{H^{\prime}}\right)=3$.

Since $d_{2}\left(G_{1}\right)+d_{3}\left(G_{1}\right)>d_{2}(G)+d_{3}(G), H^{\prime}$ contains a 5 -vertex of $G$ and no 2 or 3 -vertices of $G$. Therefore, $u \notin H^{\prime}$ and $y z \in H^{\prime}$. By part (ii) and computing $F\left(H^{\prime}\right)$ by using Lemma 3.2.8 (i), $2 F\left(H^{\prime}\right)=2 d_{2, G}\left(H^{\prime}\right)+d_{3, G}\left(H^{\prime}\right)-d_{5, G}\left(H^{\prime}\right)+3-4=-2$. By Lemma 3.2.8 (ii), $F\left(H^{\prime}-y z\right) \leq F\left(H^{\prime}\right)+1=0$. Thus $H^{\prime}-y z$ is a collapsible subgraph of $G$, contrary to that $G$ is reduced.

Case 2: $d\left(v_{H^{\prime}}\right)=2$.

Then $H^{\prime}$ contains no vertex of degree 2 or 3 in $G$. Since the number of odd degree vertices of $T(G) / H^{\prime}$ must be even, $H^{\prime}$ contains no 5 -vertex of $G$. Therefore, $2 F\left(H^{\prime}\right)=$ $2 d_{2, G}\left(H^{\prime}\right)+d_{3, G}\left(H^{\prime}\right)-d_{5, G}\left(H^{\prime}\right)+2-4=-2$. So again, $F\left(H^{\prime}-y z\right)=0$, a contradiction.

$$
\text { Hence, } d_{2}\left(G_{1}\right)+d_{3}\left(G_{1}\right) \leq d_{2}(G)+d_{3}(G) \leq 6 \text {. }
$$


Lemma 3.2.13 If $G$ is a counterexample of Theorem 3.2.1 with $|V(G)|$ minimized, then no vertex in $D_{2}(G)$ is adjacent to a vertex in $D_{4}(G)$.

Proof. By the hypothesis, $G$ is a 2-edge-connected reduced graph which satisfies $d_{2}(G)+$ $d_{3}(G) \leq 6$ and $d_{3}(G)+d_{5}(G) \leq 2$, and $G$ is neither supereulerian nor in $\mathcal{F}^{\prime}$. Since $G$ is reduced,

$$
G \text { has no nontrivial collapsible subgraphs. }
$$

Therefore, by Lemma 1.2.2(vi),

$$
G \text { has no } K_{3,3}-e \text {. }
$$

By contradiction, we assume that there exist $u \in D_{2}(G)$ and $v \in D_{4}(G)$ such that $u v \in E(G)$. We use notations in Lemma 3.2.12, and denote $G^{\prime}=T(G)$. Then $G^{\prime} \notin \mathcal{S}$ by Lemma 3.2.12(iii) and $a\left(G^{\prime}\right) \leq 2$ by Lemma 3.2.12(ii). We will prove that either $G \in \mathcal{S}$ or $G \in \mathcal{F}^{\prime}$.

Suppose $G_{1}$ is the reduction of $G^{\prime}$. Then $G_{1} \notin \mathcal{S}$, and by Lemma 3.2.12(vi) $G_{1} \in \mathcal{A}$. Since $G$ is minimized and $\left|V\left(G_{1}\right)\right| \leq\left|V\left(G^{\prime}\right)\right|=|V(G)|-1, G_{1} \in \mathcal{F}^{\prime}$. There are three cases, depending on the number of nontrivial collapsible subgraph in $G^{\prime}$ by Lemma 3.2.12(iv).

Case 1: $G^{\prime}$ doesn't have a nontrivial collapsible subgraph, i.e. $G_{1}=G^{\prime}$.

If $G^{\prime} \in\left\{K_{2,3}, K_{2,5}, S(1,2), S(2,3), S(1,4)\right\}$, no matter how we choose $y$ and $z$, the vertices $u, x, y, z$ will be in a $C_{4}$ or $C_{5}$ in $G^{\prime}$. Then in $G$, at least two of them are adjacent, contrary to Lemma 3.2.12(v).

Suppose $G^{\prime}=J(2,2)$. A trail in $G^{\prime}$ with first edge $e_{1}$ and last edge $e_{2}$ is called an $\left(e_{1}, e_{2}\right)$-trail. Note that the cycle of $G^{\prime}$ is of length 4 or 6 . If the shortest (ux,yz)-trail in $G^{\prime}$ is of length 3 or less, then at least two of $u, x, y, z$ are adjacent in $G$, contrary to Lemma 3.2.12(v). So the shortest (ux,yz)-trail is of length 4. Therefore, $u x$ and $y z$ are in a $C_{6}$. By symmetry, there are two possibilities (see Figure 3.3(a) and (b)). But both of them are supereulerian, contrary to $G \notin \mathcal{S}$. 

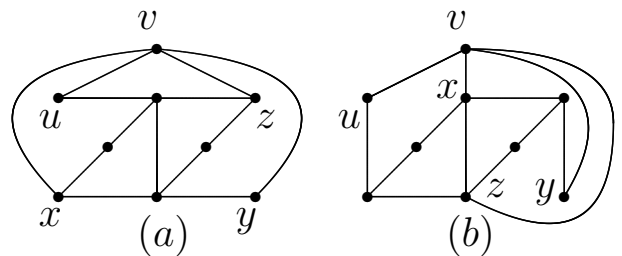

Figure 3.3. The graphs in the proof of Case 1.

Case 2: $G^{\prime}$ has only one collapsible subgraph, say $H^{\prime}$.

Let $H$ be the subgraph of $G$ corresponding to $H^{\prime}$, i.e. $T(G[E(H)])=H^{\prime}$. Then $u x$ and $y z$ are not both in $H^{\prime}$. Otherwise, $G / H \in \mathcal{F}^{\prime}$. By Lemma 3.2.10(ii), $F(H) \leq 1$. Since $\kappa^{\prime}(H) \geq 2$, by Theorem 1.2.5, $H$ is collapsible, contrary to (3.3).

Since $G_{1}=G^{\prime} / H^{\prime} \in \mathcal{F}^{\prime}$, by Lemma 3.2.10(i) and Lemma 3.2.12(ii), $F\left(H^{\prime}\right)=2\left|V\left(H^{\prime}\right)\right|-$ $\left|E\left(H^{\prime}\right)\right|-2 \leq 1$. We consider two subcases.

Subcase 2.1: $y z \in H^{\prime}$.

Then $u x$ is not in $H^{\prime}$. Since $\kappa^{\prime}\left(H^{\prime}\right) \geq 2$ and $d(u)=2, u$ is not in $H^{\prime}$. But $x$ may or may not be in $H^{\prime}$. If $x$ is in $H^{\prime}$, then $|V(H)|=\left|V\left(H^{\prime}\right)\right|+1$ and $|E(H)|=\left|E\left(H^{\prime}\right)\right|+2$. So $F(H)=2|V(H)|-|E(H)|-2=2\left|V\left(H^{\prime}\right)\right|-\left|E\left(H^{\prime}\right)\right|-2 \leq 1$. As $\kappa^{\prime}(H) \geq 2$, by Theorem 1.2.5, $H$ is collapsible, contrary to (3.3).

Then $x$ is not in $H^{\prime}$. Then $|V(H)|=\left|V\left(H^{\prime}\right)\right|+1$ and $|E(H)|=\left|E\left(H^{\prime}\right)\right|+1$. So $F(H)=\left(2\left|V\left(H^{\prime}\right)\right|-\left|E\left(H^{\prime}\right)\right|-2\right)+1 \leq 2$. Since $H$ is not collapsible and $\kappa^{\prime}(H) \geq 2$, $F(H)=2$. It implies that $H=K_{2, t}$ for some $t$. Therefore, $H^{\prime}=H-\{y v, v z\}+y z$. By the definition of $F\left(H^{\prime}\right), F\left(H^{\prime}\right)=1$. By Lemma 3.2.10(i), $H^{\prime}$ must be of Type A, B, C, D, E or F (see Table 3.3) and $G_{1} \in\left\{K_{2,3}, K_{2,5}\right\}$. Since $x$ and $u$ are not in $H, v$ is of degree 2 in $H$. Then both $y$ and $z$ are $t$-vertices in $H$ with $2 \leq t \leq 5$.

Notice that $t \neq 5$. Otherwise, $d_{G}(y)=d_{G}(z)=5$ since $d_{G}=0$ for all $j \geq 6$. That $G^{\prime} / H^{\prime} \in\left\{K_{2,3}, K_{2,5}\right\}$ and $y, z \in H^{\prime}$ implies that there is at least another 3 or 5 -vertex except $y$ and $z$, contrary to $d_{3}(G)+d_{5}(G) \leq 2$. Hence, $2 \leq t \leq 4$.

Subcase 2.1.1: $H^{\prime}$ is of Type A. 
Notice that $d_{2, G}(H)=d_{2, G^{\prime}}\left(H^{\prime}\right), d_{3, G}(H)=d_{3, G^{\prime}}\left(H^{\prime}\right)$ and $d_{5, G}(H)=d_{5, G^{\prime}}\left(H^{\prime}\right)$, so $H$ has two vertices of degree 2 in $G$ and other vertices of $H$ are of degree 4 in $G$. Since $d_{G}(u)=2$ and $u x \in G_{1}, x$ is a vertex of degree 3 in both $G_{1}$ and $G$. If $H=K_{2,2}$, then by Lemma 3.2.12(v), $d_{G}(y)=d_{G}(z)=2$, so $G \in \mathcal{S}$ (see Figure 3.4(a) and (b)). If $H=K_{2,3}$, then one of $y$ and $z$ is adjacent to $x$ (see Figure 3.4(c)), contrary to Lemma 3.2.12(v). If $H=K_{2,4}$, then $G[s, t, v, x, y, z]$ is $K_{3,3}-e$ (see Figure 3.4(d) and (e)), contrary to (3.4).

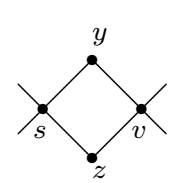

(a)

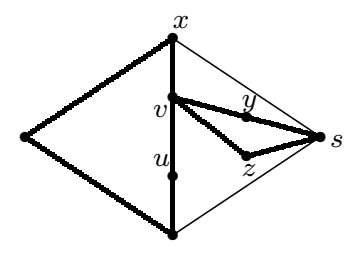

(b)

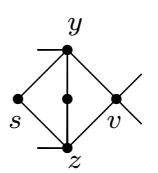

(c)

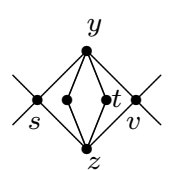

(d)

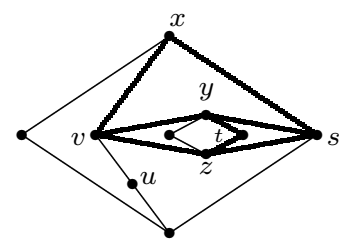

(e)

Figure 3.4. The graphs in the proof of Subcases 2.1.1 and 2.1.4.

Subcase 2.1.2: $H^{\prime}$ is of Type B.

Then $H$ has one 2-vertex, one 3 -vertex and other vertices are of degree 4 in $G$. If $H=K_{2,2}$, then $G \in \mathcal{S}$ (see Figure 3.5(a) and (b)) or $G=J(2,2)$ (see Figure 3.5(c) and (d)). If $H=K_{2,3}$ (see Figure 3.5(e) and (f)), since $H^{\prime}$ is of type B, $H^{\prime}$ has a 2-vertex in $G$. Let this vertex be $t$. Then $t$ is adjacent to $y, z$. So $t$ is not adjacent to $u$. Without loss of generality, assume $y$ is the 3 -vertex in $G$, and so $z$ is a 4 -vertex in $G$. Let $s \in N(y) \cap N(z)$ be another 2-vertex in $H^{\prime}$. By Lemma 3.2.12(v), $y, z$, are not adjacent to $u$. Since $v_{H^{\prime}}$ is adjacent to $u$, but $y, z, t$ are not adjacent to $u$, we have that $s$ is adjacent to $u$. Moreover, $v$ is also adjacent to $u$ in $G$. Therefore, $G[s, t, u, v, y, z]$ is $K_{3,3}-e$, contrary to (3.4). If $H=K_{2,4}$ (see Figure 3.5(g)), then exactly one of $s$ and $t$ is adjacent to $u$. So $G[s, t, u, v, y, z]$ is $K_{3,3}-e$, contrary to (3.4).

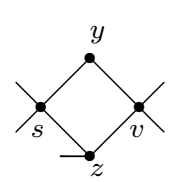

(a)

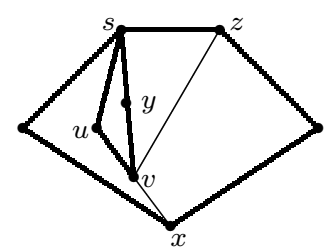

(b)

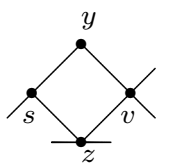

(c)

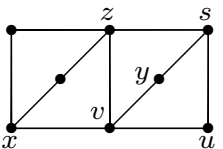

$(d)$ 


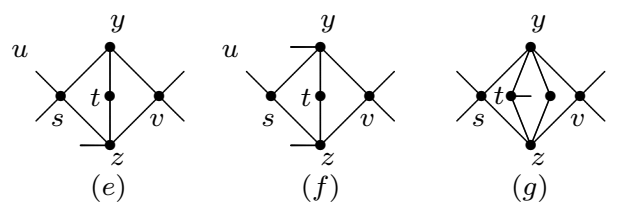

Figure 3.5. The graphs in the proof of Subcase 2.1.2.

Subcase 2.1.3: $H^{\prime}$ is of Type C.

Then $H$ has two 2-vertices, one 5 -vertex and other vertices are of degree 4 in $G$. If $H=K_{2,2}$, by Lemma 3.2.12(v), $d_{G}(s)=5$ (see Figure 3.6(a)), then $G=S(2,3)$ (see Figure 3.6(b)). If $H=K_{2,3}$ (see Figure 3.6(c)), then $y$ or $z$ is adjacent to $u$, contrary to Lemma 3.2.12(v). If $H=K_{2,4}$ (see Figure 3.6(d)), since $s$ is adjacent to $u, G[s, t, u, v, y, z]$ is $K_{3,3}-e$, contrary to (3.4).

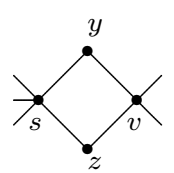

(a)

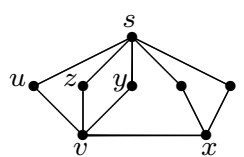

(b)

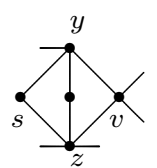

(c)

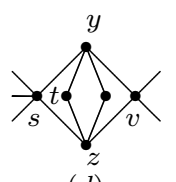

(d)

Figure 3.6. The graphs in the proof of Subcase 2.1.3.

Subcase 2.1.4: $H^{\prime}$ is of Type D.

Similar to Subcase 2.1.1, if $H=K_{2,2}$ (see Figure 3.4(a)), then $G \in \mathcal{S}$ (see Figure 3.7(a)). If $H=K_{2,3}$, then one of $y$ and $z$ is adjacent to $x$ (see Figure 3.4(c)), contrary to Lemma 3.2.12(v). If $H=K_{2,4}$ (see Figure 3.4(d)), then $G[s, t, v, x, y, z]$ is $K_{3,3}-e$, contrary to (3.4). 


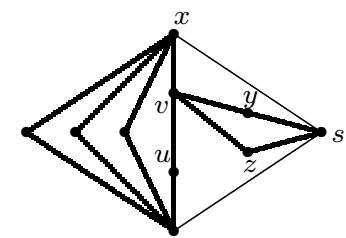

(a)

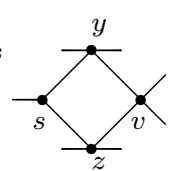

(b)

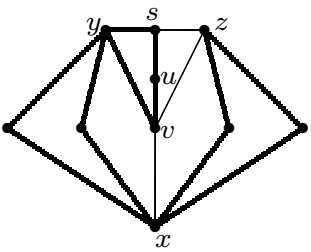

(c)

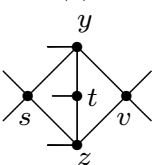

$(g)$

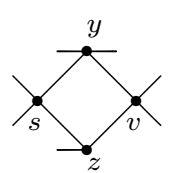

$(d)$

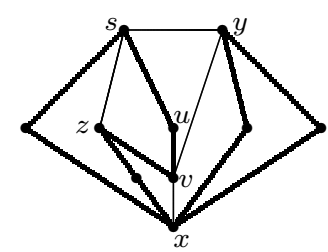

$(e)$

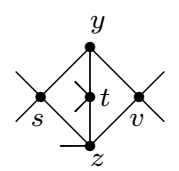

$(f)$

Figure 3.7. The graphs in the proof of Subcases 2.1.4 and 2.1.5.

Subcase 2.1.5: $H^{\prime}$ is of Type E.

If $H=K_{2,2}$, then there are two possibilities (see Figure 3.7(b) and (d)). In either case, $G \in \mathcal{S}$ (see Figure 3.7(c) and (e)). If $H=K_{2,3}$ (see Figure 3.7(f) and (g)), then $u$ is adjacent to exactly one of $s$ and $t$. Therefore, $G[s, t, u, v, y, z]$ is $K_{3,3}-e$, contrary to (3.4). If $H=K_{2,4}$ (see Figure 3.7(h)), then $u$ is adjacent to exactly one of $s, t$ and $w$. Assume that $u$ is adjacent to $s$. Then $G[s, t, u, v, y, z]$ is $K_{3,3}-e$, contrary to (3.4).

Subcase 2.1.6: $H^{\prime}$ is of Type F.

If $H=K_{2,2}$ (see Figure 3.8(a) and (c)), then $G \in \mathcal{S}$ (see Figure 3.8(b) and (d)). If $H=K_{2,3}$ (see Figure 3.8(e)) or $H=K_{2,4}$ (see Figure 3.8(f)), then $G[s, t, u, v, y, z]$ is $K_{3,3}-e$, contrary to $(3.4)$.

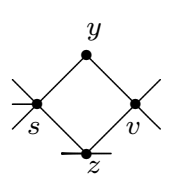

(a)

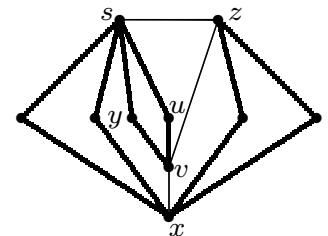

(b)
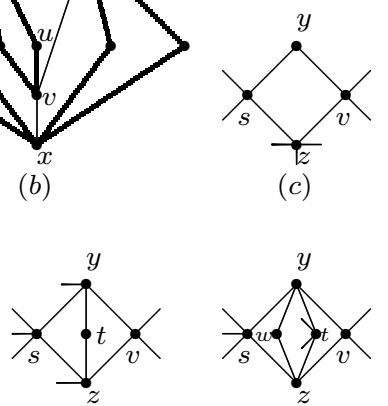

(e)

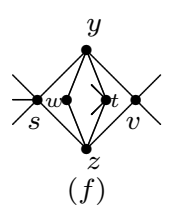

$(f)$

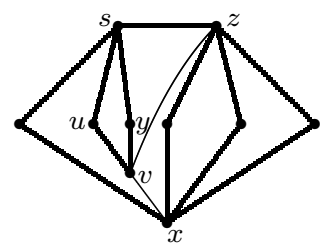

$(d)$

Figure 3.8. The graphs in the proof of Subcase 2.1.6. 
Subcase 2.2: $u x \in H^{\prime}$

Similar to Subcase 2.1, if $y$ or $z$ is in $H^{\prime}$, then $|V(H)|=\left|V\left(H^{\prime}\right)\right|+1$ and $|E(H)|=$ $\left|E\left(H^{\prime}\right)\right|+2$. Therefore, $F(H) \leq 1$. So $y$ and $z$ are not in $H^{\prime}, F\left(H^{\prime}\right)=1$ and $H=K_{2, t}$. Since $u$ is a 2 -vertex, $d_{2}\left(H^{\prime}\right)>1$ and $t=2$. So $H^{\prime}$ must be of Type A, B, C, D or F and $H$ is $K_{2,2}$. Use the same argument to conclude that $G \in \mathcal{S}$ or $G \in\{J(2,2), S(2,3)\}$.

Case 3: $G^{\prime}$ has two nontrivial maximal collapsible subgraphs, say $H_{1}^{\prime}$ and $H_{2}^{\prime}$, such that $y z \in H_{1}^{\prime}$ and $u x \in H_{2}^{\prime}$.

Let $H_{1}$ and $H_{2}$ be the subgraphs of $G$ corresponding to $H_{1}^{\prime}$ and $H_{2}^{\prime}$, respectively, i.e. $T\left(G\left[E\left(H_{1}\right)\right]\right)=H_{1}^{\prime}$ and $T\left(G\left[E\left(H_{2}\right)\right]\right)=H_{2}^{\prime}$. Then $G^{\prime} /\left(H_{1}^{\prime} \cup H_{2}^{\prime}\right)$ is in $\mathcal{F}^{\prime}$. Notice that $v_{H_{1}^{\prime}} \neq v_{H_{2}^{\prime}}$. Otherwise, there exists a vertex $t$ such that $t \in V\left(H_{1}^{\prime}\right) \bigcap V\left(H_{2}^{\prime}\right)$. Then $H_{1}^{\prime} \cup H_{2}^{\prime}$ is a connected collapsible subgraph of $G^{\prime}$, contrary to that $H_{1}^{\prime}$ and $H_{2}^{\prime}$ are maximal.

Let $n^{\prime}$ denote the number of vertices of $H_{1} \cup H_{2}, d_{i}^{\prime}$ denote the number of vertices of $H_{1} \cup H_{2}$ of degree $i$ in $G$. Then $2\left|E\left(H_{1} \cup H_{2}\right)\right|=\sum i d_{i}^{\prime}-d\left(v_{H_{1}^{\prime}}\right)-d\left(v_{H_{2}^{\prime}}\right)$. Since $v$ is in both $H_{1}$ and $H_{2},\left|V\left(H_{1}\right)\right|+\left|V\left(H_{2}\right)\right|=n^{\prime}+1$.

$$
\begin{aligned}
2 F\left(H_{1}\right)+2 F\left(H_{2}\right) & =4\left|V\left(H_{1}\right)\right|-2\left|E\left(H_{1}\right)\right|-4+4\left|V\left(H_{2}\right)\right|-2\left|E\left(H_{2}\right)\right|-4 \\
& =4\left(\left|V\left(H_{1}\right)\right|+\left|V\left(H_{2}\right)\right|\right)-2\left(\left|E\left(H_{1}\right)\right|+\left|E\left(H_{2}\right)\right|-8\right. \\
& =4\left(n^{\prime}+1\right)-2\left|E\left(H_{1} \cup H_{2}\right)\right|-8 \\
& =4\left(\sum d_{i}^{\prime}+1\right)-\left(\sum i d_{i}^{\prime}-d\left(v_{H_{1}^{\prime}}\right)-d\left(v_{H_{2}^{\prime}}\right)\right)-8 \\
& \leq 2 d_{2}^{\prime}+d_{3}^{\prime}-d_{5}^{\prime}+d\left(v_{H_{1}^{\prime}}\right)+d\left(v_{H_{2}^{\prime}}\right)-4 .
\end{aligned}
$$

By Lemma 3.2.3 and $G \notin \mathcal{S}, F(G)=2$ or $F(G)=3$ with $d_{2}(G)+d_{3}(G)=6$ and $d_{3}(G)+d_{5}(G)=2$. If $F(G)=2$, by Theorem 1.2.6, since $d_{2}(G)+d_{3}(G) \leq 6, \kappa^{\prime}(G) \geq 2$ and $G \notin \mathcal{S}, G=K_{2,3}$ or $K_{2,5}$, contrary to $G \notin \mathcal{F}^{\prime}$. Thus $F(G)=3$ with $d_{2}(G)+d_{3}(G)=6$ and $d_{3}(G)+d_{5}(G)=2$. We have the following Table 3.4, where $\left\{d\left(H_{1}^{\prime}\right), d\left(H_{2}^{\prime}\right)\right\}$ is a multiset and $G_{1}=G^{\prime} /\left(H_{1}^{\prime} \bigcup H_{2}^{\prime}\right) \in \mathcal{F}^{\prime}$. Note that $H_{2}^{\prime}$ contains a 2-vertex $u$, so $d_{2}^{\prime} \geq 1$. It helps us get rid of some cases. 


\begin{tabular}{|c|c|c|c|c|c|}
\hline$G_{1}$ & $\left\{d\left(v_{H_{1}^{\prime}}\right), d\left(v_{H_{2}^{\prime}}\right)\right\}$ & $d_{2}^{\prime}$ & $d_{3}^{\prime}$ & $d_{5}^{\prime}$ & $F\left(H_{1}\right)+F\left(H_{2}\right)$ \\
\hline \multirow[t]{6}{*}{$K_{2,3}$} & $\{2,2\}$ & 3 & 0 & 0 & 3 \\
\hline & $\{2,3\}$ & 2 & 1 & 0 & 3 \\
\hline & & 3 & 0 & 1 & 3 \\
\hline & $\{3,3\}$ & 1 & 2 & 0 & 3 \\
\hline & & 2 & 1 & 1 & 3 \\
\hline & & 3 & 0 & 2 & 3 \\
\hline \multirow[t]{4}{*}{$K_{2,5}$} & $\{2,2\}$ & 3 & 0 & 0 & 3 \\
\hline & $\{2,5\}$ & 1 & 1 & 0 & 3 \\
\hline & & 2 & 0 & 1 & 3 \\
\hline & $\{5,5\}$ & 1 & 0 & 2 & 3 \\
\hline \multirow[t]{7}{*}{$S(2,3)$} & $\{2,2\}$ & 2 & 0 & 0 & 2 \\
\hline & $\{2,3\}$ & 1 & 1 & 0 & 2 \\
\hline & & 2 & 0 & 1 & 2 \\
\hline & $\{2,4\}$ & 1 & 0 & 0 & 2 \\
\hline & $\{2,5\}$ & 1 & 0 & 1 & 2 \\
\hline & $\{3,4\}$ & 1 & 0 & 1 & 2 \\
\hline & $\{3,5\}$ & 1 & 0 & 2 & 2 \\
\hline \multirow[t]{7}{*}{$J(2,2)$} & $\{2,2\}$ & 2 & 0 & 0 & 2 \\
\hline & $\{2,3\}$ & 1 & 1 & 0 & 2 \\
\hline & & 2 & 0 & 1 & 2 \\
\hline & $\{2,4\}$ & 1 & 0 & 0 & 2 \\
\hline & $\{3,3\}$ & 1 & 1 & 1 & 2 \\
\hline & & 2 & 0 & 2 & 2 \\
\hline & $\{3,4\}$ & 1 & 0 & 1 & 2 \\
\hline
\end{tabular}

Table 3.4. The table in the proof of Case 3.

If $G_{1}=S(1,2)$, then since $S(1,2)$ has one more 2-vertex than $K_{2,3}$, the number of 2-vertices in $H_{1} \cup H_{2}$ will decrease by 1 comparing to the case $G_{1}=K_{2,3}$. Therefore, $F\left(H_{1}\right)+F\left(H_{2}\right)$ decreases by 1 . Hence, $F\left(H_{1}\right)+F\left(H_{2}\right) \leq 3-1=2$. If $G_{1}=S(1,4)$, then since $S(1,4)$ has one more 2-vertex than $K_{2,5}, F\left(H_{1}\right)+F\left(H_{2}\right)$ will decrease by 1 comparing to the case $G_{1}=K_{2,5}$. Thus, $F\left(H_{1}\right)+F\left(H_{2}\right) \leq 3-1=2$. So we have 
$F\left(H_{1}\right)+F\left(H_{2}\right) \leq 3$ (see Table 3.4). Thus $F\left(H_{1}\right) \leq 1$ or $F\left(H_{2}\right) \leq 1$. Since $\kappa^{\prime}\left(H_{i}\right) \geq 2$ for $i=1,2$, then $H_{1}$ or $H_{2}$ is a collapsible subgraph of $G$, contrary to (3.3).

Proof of Theorem 3.2.1: By contradiction, suppose $G$ satisfies (i) and (ii), but $G \notin$ $\mathcal{S}$ and $G \notin \mathcal{F}^{\prime}$ with $|V(G)|$ minimized. By Lemma 3.2.3, Theorem 1.2.6 and $G \notin$ $\left\{K_{2,3}, K_{2,5}\right\}, F(G)=3$. Therefore, $G \in \mathcal{A}_{3}$. By Lemma 3.2.4, $\left(d_{2}, d_{3}, d_{5}\right) \in\{(4,2,0),(5,1,1),(6,0,2)\}$. By Lemmas 3.2.5, 3.2.9 and 3.2.13, each vertex in $D_{2}(G)$ must be adjacent to two odd degree vertices which are not adjacent. But this is impossible when $\left(d_{2}, d_{3}, d_{5}\right) \in$ $\{(4,2,0),(5,1,1),(6,0,2)\}$.

Thus the theorem holds.

\subsection{Proof of the Main Result}

In this section, we are now ready to prove our main result Theorem 3.1.6.

Proof. Let $G \in C_{2}(6, k)$ be a graph with $n=|V(G)|>7 k$. Then we will prove that $G \in \mathcal{S}$ if and only if $G$ can not be contracted to a member in $\mathcal{F}^{\prime}$. Clearly, if $G$ can be contracted to a member in $\mathcal{F}^{\prime}$, then $G \notin \mathcal{S}$.

Let $G^{\prime}$ be the reduction of $G$. By Theorem 1.2.2(ii), it suffices to show if $G^{\prime} \notin \mathcal{S}$, then $G^{\prime} \in \mathcal{F}^{\prime}$, which implies that $G$ can be contracted to a member in $\mathcal{F}^{\prime}$. As $G^{\prime}=K_{1}$ implies that $G \in \mathcal{S}$, we may assume that $G^{\prime}$ is 2-edge-connected and nontrivial. Let $d_{i}^{\prime}=\left|d_{i}\left(G^{\prime}\right)\right|$.

By Theorem 1.2.7, if $d_{2}^{\prime}+d_{3}^{\prime}=4$, then $G^{\prime} \in \mathcal{S}$. Therefore, we only consider the case when $d_{2}^{\prime}+d_{3}^{\prime} \geq 5$. We shall assume that $G^{\prime} \notin \mathcal{S}$ to find a contradiction or to get $G^{\prime} \in \mathcal{F}^{\prime}$.

Case 1: $d_{2}^{\prime}+d_{3}^{\prime}=5$.

Subcase 1.1: $F\left(G^{\prime}\right) \leq 2$.

By Theorem 1.2.6, since $\kappa^{\prime}\left(G^{\prime}\right) \geq 2$ and $G^{\prime} \notin \mathcal{S}, G^{\prime}=K_{2, t}$ with $t$ odd. Since 
$d_{2}^{\prime}+d_{3}^{\prime}=5$, we have $t=3$ or $t=5$ and so $G^{\prime} \in\left\{K_{2,3}, K_{2,5}\right\} \subset \mathcal{F}^{\prime}$.

Subcase 1.2: $F\left(G^{\prime}\right) \geq 3$.

By Theorem 1.2.2(v), we have

$$
\begin{aligned}
6 \leq 2 F\left(G^{\prime}\right) & =4\left|V\left(G^{\prime}\right)\right|-2\left|E\left(G^{\prime}\right)\right|-4 \\
& =4 \sum_{j \geq 2} d_{j}^{\prime}-\sum_{j \geq 2} j d_{j}^{\prime}-4 \\
& =\left(d_{2}^{\prime}+d_{3}^{\prime}\right)+d_{2}^{\prime}+\sum_{j \geq 5}(4-j) d_{j}^{\prime}-4 \\
& =1+d_{2}^{\prime}+\sum_{j \geq 5}(4-j) d_{j}^{\prime} .
\end{aligned}
$$

Note that $d_{2}^{\prime}+d_{3}^{\prime}=5$ and $(4-j) d_{j}^{\prime} \leq 0$ for any $j \geq 5$. It follows that $d_{2}^{\prime}=5, d_{3}^{\prime}=0$, and $d_{j}^{\prime}=0(j \geq 5)$. Thus $G^{\prime}$ is Eulerian contrary to that $G^{\prime} \notin \mathcal{S}$.

Case 2: $d_{2}^{\prime}+d_{3}^{\prime}=6$.

If $F\left(G^{\prime}\right) \leq 2$, then by $\kappa^{\prime}\left(G^{\prime}\right) \geq 2$ and by Theorem 1.2.6, $G^{\prime}=K_{2, t}$ with $t \geq 3$ odd since $G^{\prime}$ is not supereulerain. As $d_{2}^{\prime}+d_{3}^{\prime}=6$, this is impossible. Therefore, we must have $F\left(G^{\prime}\right) \geq 3$.

Subcase 2.1: $F\left(G^{\prime}\right)=3$.

$$
\begin{aligned}
6=2 F\left(G^{\prime}\right) & =4\left|V\left(G^{\prime}\right)\right|-2\left|E\left(G^{\prime}\right)\right|-4 \\
& =4 \sum_{j \geq 2} d_{j}^{\prime}-\sum_{j \geq 2} d_{j}^{\prime}-4 \\
& =2\left(d_{2}^{\prime}+d_{3}^{\prime}\right)-\left(d_{3}^{\prime}+d_{5}^{\prime}\right)+\sum_{j \geq 6}(4-j) d_{j}^{\prime}-4 \\
& =8-\left(d_{3}^{\prime}+d_{5}^{\prime}\right)+\sum_{j \geq 6}(4-j) d_{j}^{\prime} .
\end{aligned}
$$

It follows that $\left(d_{3}^{\prime}+d_{5}^{\prime}\right) \leq 2$. By Theorem 3.2.1, since $G^{\prime} \notin \mathcal{S}$, we have $G^{\prime} \in \mathcal{F}^{\prime}$.

Subcase 2.2: $F\left(G^{\prime}\right) \geq 4$. 
Since $d_{2}^{\prime}+d_{3}^{\prime}=6$,

$$
\begin{aligned}
8 \leq 2 F\left(G^{\prime}\right) & =\left(d_{2}^{\prime}+d_{3}^{\prime}\right)+d_{2}^{\prime}+\sum_{j \geq 5}(4-j) d_{j}^{\prime}-4 \\
& =2+d_{2}^{\prime}+\sum_{j \geq 5}(4-j) d_{j}^{\prime}
\end{aligned}
$$

It follows that $d_{2}^{\prime}=6, d_{3}^{\prime}=0$ and $d_{j}^{\prime}=0(j \geq 5)$. Hence $G^{\prime}$ is Eulerian, contrary to $G^{\prime} \notin \mathcal{S}$.

Case 3: $d_{2}^{\prime}+d_{3}^{\prime} \geq 7$.

Let $c=d_{2}^{\prime}+d_{3}^{\prime}$, and $H_{1}, H_{2}, \cdots, H_{c}$ denote the subgraphs of $G$ whose contraction images in $G^{\prime}$ are the vertices of degree at most 3 in $G^{\prime}$. Since $G \in C_{2}(6, k)$, for each $i$ with $1 \leq i \leq c,\left|V\left(H_{i}\right)\right| \geq(n-k) / 6$. It follows any $c \geq 7$ that

$$
n=|V(G)| \geq \sum_{i=1}^{7}\left|V\left(H_{i}\right)\right| \geq \frac{7(n-k)}{6} .
$$

Therefore, $n \leq 7 k$, a contradiction.

This completes the proof of Theorem 3.1.6. 


\section{Chapter 4}

\section{Characterization of Graphs with Mod $(2 p+1)$-orientations}

\subsection{Some Useful Facts}

We consider finite graphs without loops, but multiple edges are allowed. If $H_{1}$ and $H_{2}$ are subgraphs of a graph $G$, then $H_{1} \cap H_{2}$ and $H_{1} \cup H_{2}$ are the intersection and the union of $H_{1}$ and $H_{2}$, respectively, as defined in [1].

In this section, we introduce the mod $(2 p+1)$-closure of a graph, and investigate the distribution of the in-degrees and out-degrees of certain vertices in a graph with a mod $(2 p+1)$-orientation. We start with a lemma.

Lemma 4.1.1 Let $G$ be a graph, and $m \geq 1$ be an integer. Each of the following holds.

(i) If $G \in M_{2 p+1}^{o}$, then $\kappa^{\prime}(G) \geq 2 p$.

(ii) $m K_{2} \in M_{2 p+1}^{o}$ if and only if $m \geq 2 p$.

Proof. (i). We argue by contradiction and assume that $G$ is in $M_{2 p+1}^{o}$ with $\kappa^{\prime}(G)<2 p$. Then $G$ has an edge cut $X$ with $|X|<2 p$. Let $G_{1}, G_{2}$ denote the two components of 
$G-X$. By Proposition 1.3.3(C2), $G^{\prime}=G / G_{1} \in M_{2 p+1}^{o}$. Let $v$ denote the vertex of $G^{\prime}$ onto which $G_{1}$ is contracted. Then $d_{G^{\prime}}(v)=|X|<2 p$.

Suppose first that $d_{G^{\prime}}(v)=2 k<2 p$. Pick a map $b \in Z\left(G^{\prime}, \mathbb{Z}_{2 p+1}\right)$ with $b(v)=1$. As $G^{\prime} \in M_{2 p+1}^{o}, G^{\prime}$ has a $\left(b, \mathbb{Z}_{2 p+1}\right)$-orientation $D=D\left(G^{\prime}\right)$. Under this orientation, $d^{+}(v)+d^{-}(v)=2 k$ and $d^{+}(v)-d^{-}(v) \equiv 1(\bmod 2 p+1)$. It follows by $k<p$ that $2 d^{+}=2 k+1$, a contradiction.

Next we assume that $d_{G^{\prime}}(v)=2 k+1<2 p$. Pick a map $b \in Z\left(G^{\prime}, \mathbb{Z}_{2 p+1}\right)$ with $b(v)=0$. As $G^{\prime} \in M_{2 p+1}^{o}, G^{\prime}$ has a $\left(b, \mathbb{Z}_{2 p+1}\right)$-orientation $D=D\left(G^{\prime}\right)$. Under this orientation, $d^{+}(v)+d^{-}(v)=2 k+1$ and $d^{+}(v)-d^{-}(v) \equiv 0(\bmod 2 p+1)$. It follows by $k<p$ that $2 d^{+}=2 k+1$, a contradiction.

(ii). First assume that $m=2 p$. By Part (i), it suffices to show that $m K_{2} \in M_{2 p+1}^{o}$. Let $V\left(m K_{2}\right)=\left\{v_{1}, v_{2}\right\}$, and $b\left(v_{1}\right)=b^{\prime}$ with $0 \leq b^{\prime} \leq m$. Then exactly one in $\left.\left\{m-b^{\prime}, b^{\prime}-1\right)\right\}$ is an even number $2 t$ with $0 \leq t \leq p$. Orient $m K_{2}$ such that exactly $t$ edge is directed from $v_{2}$ to $v_{1}$ if $m-b^{\prime}$ is even; or such that exactly $t$ edge is directed from $v_{1}$ to $v_{2}$ if $b^{\prime}-1$ is even. This yields a $\left(\mathbb{Z}_{2 p+1}, b\right)$-orientation of $m K_{2}$, and so $m K_{2} \in M_{2 p+1}^{o}$. If $m \geq 2 p+1$, then $m K_{2} /\left((2 p) K_{2}\right)=K_{1} \in M_{2 p+1}^{o}$, and so by Proposition 1.3.3(C3), $m K_{2} \in M_{2 p+1}^{o}$. This completes the proof of the lemma.

Definition 4.1.2 Let $H$ be a subgraph of $G$, and let $p>0$ be an integer. The mod $(2 p+1)$-closure of $H$ in $G$, denoted by $c l_{G}^{2 p+1}(H)$, or $c l(H)$ when $G$ and $p$ is understood from the context, is a maximal subgraph of $G$ satisfying (i) and (ii) below.

(i) $H \subseteq c l(H)$, and

(ii) If $H^{\prime} \subseteq c l(H)$ and if $v \in V(G)-V\left(H^{\prime}\right)$ such that $\left|\left[V\left(H^{\prime}\right),\{v\}\right]\right| \geq 2 p$, then $G\left[V\left(H^{\prime}\right) \cup\right.$ $\{v\}] \subseteq \operatorname{cl}(H)$.

From definition 4.1.2, if $\operatorname{cl}(H) \neq H$, then $V(\operatorname{cl}(H))=V(H) \cup\left\{v_{1}, v_{2}, \cdots, v_{t}\right\}$ such that $V(H) \cap\left\{v_{1}, v_{2}, \cdots, v_{t}\right\}=\varnothing$, and such that for each $i$ with $1 \leq i \leq t-1$,

$$
\left|\left[\left\{v_{i+1}\right\}, V(H) \cup\left\{v_{1}, v_{2}, \cdots, v_{i}\right\}\right]\right| \geq 2 p .
$$

The sequence $\left(v_{1}, v_{2}, \cdots, v_{t}\right)$ satisfying (4.1) will be referred as a closure sequence of $H$ in $G$. 
Proposition 4.1.3 Let $H$ be a subgraph of $G$, and let $p>0$ be an integer, and let $c l(H)=c l_{G}^{2 p+1}(H)$. If $H \in M_{2 p+1}^{o}$, then each of the following holds.

(i) The closure $c l(H)$ is uniquely determined.

(ii) $\operatorname{cl}(H) \in M_{2 p+1}^{o}$.

(iii) The graph $G \in M_{2 p+1}^{o}$ if and only if $G / c l(H) \in M_{2 p+1}^{o}$.

(iv) The graph $G \in M_{2 p+1}$ if and only if $G / c l(H) \in M_{2 p+1}$.

(v) For any $n \geq 4 p+1, K_{n} \in M_{2 p+1}^{o}$.

Proof. (i). Let $H^{\prime}$ and $H^{\prime \prime}$ be two closures of $H$ in $G$. Let $\left(v_{1}^{\prime}, v_{2}^{\prime}, \cdots, v_{s}^{\prime}\right)$ be a closure sequence of $H$ in $G$ such that $V\left(H^{\prime}\right)=V(H) \cup\left\{v_{1}^{\prime}, v_{2}^{\prime}, \cdots, v_{s}^{\prime}\right\}$, and $\left(v_{1}^{\prime \prime}, v_{2}^{\prime \prime}, \cdots, v_{t}^{\prime \prime}\right)$ be a closure sequence of $H$ in $G$ such that $V\left(H^{\prime \prime}\right)=V(H) \cup\left\{v_{1}^{\prime \prime}, v_{2}^{\prime \prime}, \cdots, v_{t}^{\prime \prime}\right\}$. Then there exists a largest integer $I>0$ such that for all $1 \leq i \leq I$, both $v_{i}^{\prime \prime} \in\left\{v_{1}^{\prime}, v_{2}^{\prime}, \cdots, v_{s}^{\prime}\right\}$ and $v_{i}^{\prime} \in\left\{v_{1}^{\prime \prime}, v_{2}^{\prime \prime}, \cdots, v_{t}^{\prime \prime}\right\}$. Assume that the choice of these closure sequences corresponding to $H^{\prime}$ and $H^{\prime \prime}$ maximizes this value $I$.

As $I=s=t$ implies $H^{\prime}=H^{\prime \prime}$, we assume that for $j=I+1, v_{j}^{\prime} \in V\left(H^{\prime}\right)-V\left(H^{\prime \prime}\right)$. Let $H_{0}=G\left[V(H) \cup\left\{v_{1}^{\prime}, \cdots, v_{I}^{\prime}\right\}\right]$. Then by (4.1), $v_{j}^{\prime}$ is adjacent to at least $2 p$ vertices in $H_{0}$. By Definition 4.1.2(ii), $v_{j}^{\prime} \in V\left(H^{\prime \prime}\right)$ as well. By the choice of these closure sequences, we must have $v_{j}^{\prime}=v_{j}^{\prime \prime}$, contrary to the maximality of $I$. This proves that $V\left(H^{\prime}\right) \subseteq V\left(H^{\prime \prime}\right)$. By a similar argument, $V\left(H^{\prime \prime}\right) \subseteq V\left(H^{\prime}\right)$. Thus $V\left(H^{\prime}\right)=V\left(H^{\prime \prime}\right)$, and so $H^{\prime}=H^{\prime \prime}$.

(ii). Let $\left(v_{1}, v_{2}, \cdots, v_{t}\right)$ denote a closure sequence of $H$ in $G$. Let $H_{i}=G[V(H) \cup$ $\left.\left\{v_{1}, v_{2}, \cdots, v_{i}\right\}\right]$ with $H_{0}=H$. We argue by induction on $0 \leq i \leq t$ to show that $i \in M_{2 p+1}^{o}$. As $H \in M_{2 p+1}^{o}$, we assume that $H_{i-1} \in M_{2 p+1}^{o}$ with $i \geq 1$. By (4.1), $v_{i}$ is adjacent to $m \geq 2 p$ vertices in $H_{i-1}$. Thus $H_{i} / H_{i-1} \cong m K_{2}$ with $m \geq 2 p$, and so by Lemma 4.1.1(ii), $H_{i} / H_{i-1} \in M_{2 p+1}^{o}$. It now follows by Proposition 1.3.3(C3) that $H_{i} \in M_{2 p+1}^{o}$. By induction, $\operatorname{cl}(H)=H_{t} \in M_{2 p+1}^{o}$.

(iii) and (iv). These follow from Proposition 1.3.3(C2) and (C3), and from Proposition 4.1.3(ii) above.

(v). By Corollary 3.4 in [38], $K_{4 p+1} \in M_{2 p+1}^{o}$. When $n \geq 4 p+1$, we can view $K_{4 p+1}$ as a subgraph of $K_{n}$. Since $c l\left(K_{4 p+1}\right)=K_{n}$, (v) follows from (ii). 
Lemma 4.1.4 Let $G$ be a connected graph with a mod $(2 p+1)$-orientation D. Each of the following holds.

(i) For every vertex $v \in D_{4 p-1}(G)$, either $d_{D}^{+}(v)=3 p$ (in which case $v$ is called a positive vertex of $D)$, or $d_{D}^{+}(v)=p-1$, (in which case $v$ is called a negative vertex of $D$ ).

(ii) If $G$ is simple and if $X \subseteq D_{4 p-1}(G)$ is a set of positive (or negative) vertices of $G$ such that $G[X]$ is a complete subgraph of $G$, then $|V(G)|-|X| \geq 2 p+1$.

(iii) If $G$ is simple and if $X \subseteq D_{4 p-1}(G)$ is a set of positive (or negative) vertices of $G$ such that $G[X]$ is a complete subgraph of $G$, then $|X| \leq 2 p-1$.

Proof. (i). Let $v \in D_{4 p-1}(G), d^{+}=d_{D}^{+}(v)$ and $d^{-}=d_{D}^{-}(v)$. As $d^{+}-d^{-}=0$ implies a contradiction $2 d^{+}=4 p-1$, and as $d^{+}+d^{-}=4 p-1$, it follows that $d^{+}-d^{-} \in$ $\{2 p+1,-2 p-1\}$. If $d^{+}-d^{-}=2 p+1$, then $d^{+}=3 p$ and $d^{-}=p-1$; if $d^{+}-d^{-}=-2 p-1$, then $d^{-}=3 p$ and $d^{+}=p-1$.

(ii). We assume that there exists a set $X$ of positive vertices with $|V(G)|-|X| \leq 2 p$ such that $H^{\prime}=G[X]$ is a complete graph. Then $D^{\prime}=D\left(H^{\prime}\right)$ is a sub-digraph of $D=$ $D(G)$. At each $x \in X$, since $x$ is a positive vertex, it follows by (i) and by the assumption of $|V(G)|-|X| \leq 2 p$ that $D^{\prime}$ has at least $p$ edges directed out from $x$, and at most $p-1$ edges directed into $x$. This leads to a contradiction: $p|X| \leq\left|E\left(H^{\prime}\right)\right| \leq(p-1)|X|$.

(iii) By contradiction, we assume that $|X| \geq 2 p$. Let $X^{\prime} \subseteq X$ with $\left|X^{\prime}\right|=2 p$. Then $H^{\prime}=G\left[X^{\prime}\right]$ is a complete graph and $D^{\prime}=D\left(H^{\prime}\right)$ is a subdigraph of $D=D(G)$. At each $x \in X$, since $x$ is a positive vertex, it follows by (i) that $d_{D^{\prime}}^{-}(x) \leq p-1$ and so $p(2 p-1)=\left|E\left(H^{\prime}\right)\right|=\sum_{x \in X} d_{D^{\prime}}^{-}(x) \leq(p-1) 2 p$, a contradiction.

Example 4.1.5 Let $p \geq 1$ be an integer. As an example, we shall show that $K_{m} \notin M_{2 p+1}^{o}$ for any $m$ with $3 \leq m \leq 4 p$. This, together with Lemma 4.1.3(v), shows that a complete graph $K_{n}$ is in $M_{2 p+1}^{o}$ if and only if $n \geq 4 p+1$.

As a first step, we show that for any integer $p$ with $p>0, K_{4 p} \notin M_{2 p+1}$, and so $K_{4 p} \notin M_{2 p+1}^{o}$. Let $G=K_{4 p}$ and suppose that $G$ has a $\bmod (2 p+1)$-orientation $D=D(G)$. Let $V_{P}$ denote the set of all positive vertices of $D(G)$. By the lemma above, since $V(G)-V_{P}$ is the set of all negative vertices, $\left|V_{P}\right| \geq 2 p+1$. By the same reason, $\left|V(G)-V_{P}\right| \geq 2 p+1$, 
which leads to a contradiction:

$$
4 p=\left|V(G)-V_{P}\right|+\left|V_{P}\right| \geq 2(2 p+1)=4 p+2 .
$$

Now let $m$ be an integer with $3 \leq m \leq 4 p-1$. By Lemma 4.1.1(i), we may assume that $2 p+1 \leq m \leq 4 p-1$, and so we can view that $K_{m}$ as a subgraph $K_{4 p}$. Since $m \geq 2 p+1$, $c l_{K_{4 p}}^{2 p+1}\left(K_{m}\right)=K_{4 p}$. Thus if $K_{m} \in M_{2 p+1}^{o}$, then by Proposition 4.1.3(ii), we would have $K_{4 p} \in M_{2 p+1}^{o}$, contrary to the fact $K_{4 p} \notin M_{2 p+1}^{o}$. Hence $K_{m} \notin M_{2 p+1}^{o}$.

Lemma 4.1.6 Let $G$ be a connected graph and let $D_{2 p+1}=D_{2 p+1}(G)$. Each of the following holds.

(i) If $D$ is a $\bmod (2 p+1)$-orientation of $G$, then for any $v \in D_{2 p+1}(G)$, either $d_{D}^{+}(v)=$ $2 p+1$ or $d_{D}^{-}(v)=2 p+1$. In particular, $G\left[D_{2 p+1}\right]$ must be a bipartite graph, such that in $G\left[D_{2 p+1}\right]$, all neighbors of a vertex $u$ with $d_{D}^{+}(u)=2 p+1\left(d_{D}^{-}(u)=2 p+1\right.$, respectively) must be vertices $v$ with $d_{D}^{-}(v)=2 p+1\left(d_{D}^{+}(v)=2 p+1\right.$, respectively).

(ii) Suppose that $G$ is a $(2 p+1)$-regular graph. Then $G$ has a mod $(2 p+1)$-orientation if and only if $G$ is bipartite.

(iii) If $G$ is a bipartite graph with a vertex bipartition $(X, Y)$ such that for every vertex $x \in V(G), d_{G}(x) \equiv 0(\bmod 2 p+1)$, then $G \in M_{2 p+1}$.

(iv) If $G$ has a $\bmod (2 p+1)$-orientation, then for any $v \in V(G)$, either $d_{D}^{+}(v)=d_{D}^{-}(v)$ or $d_{G}(v) \geq 2 p+1$.

(v) If for every vertex $v \in V(G), d_{G}(v) \equiv 0(\bmod 2 p+1)$, and for some vertex $w, G-w$ is bipartite, then $G \in M_{2 p+1}$.

Proof. The verifications for (i)-(iv) are straightforward, and so will be omitted. We will only show (v). Let $(X, Y)$ denote the bipartition of $G-w$. For any $e=u v \in E(G)$, orient $e$ to a directed edge $(u, v)$ if $u \in X$ or $v \in Y$. Denote this resulting orientation by $D$. Since $\forall v \in V(G), d_{G}(v) \equiv 0(\bmod 2 p+1)$, for every $v \in V(G-w), d_{D}^{+}(v)-d_{D}^{-}(v) \equiv 0$ $(\bmod 2 p+1)$. Thus

$$
d_{D}^{-}(w)-d_{D}^{+}(w)=\sum_{v \in V(G-w)} d_{D}^{+}(v)-d_{D}^{-}(v) \equiv 0(\bmod 2 p+1),
$$

and so $D$ is a $\bmod (2 p+1)$-orientation of $G$. 


\subsection{A Characterization of Graphs with $\operatorname{Mod}(2 p+1)-$ orientations}

In this section, we will present a characterization of graphs in $M_{2 p+1}$.

Theorem 4.2.1 Let $G$ be a connected graph. Then $G$ has a mod $(2 p+1)$-orientation if and only if $G$ is the contraction of a $(2 p+1)$-regular bipartite graph.

Proof. Suppose first that $G$ is the contraction of a $(2 p+1)$-regular bipartite graph $G^{\prime}$. By Lemma 4.1.6(ii), $G^{\prime}$ has a mod (2p+1)-orientation, and so by Proposition 1.3.3(C2'), $G$ has a $\bmod (2 p+1)$-orientation.

Conversely, we assume that $G$ has a mod $(2 p+1)$-orientation. We shall fix this mod $(2 p+1)$-orientation $D$ (say) in the discussion below. Define

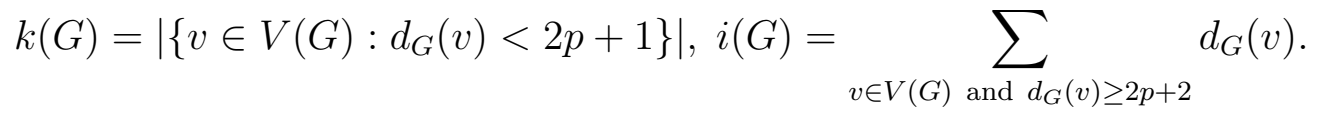

We shall argue by induction on $i(G)+k(G)$ to show that $G$ is a contraction of a $(2 p+1)$ regular bipartite graph.

If $i(G)+k(G)=0$, then $G$ is $(2 p+1)$-regular. By Lemma 4.1.6(i), $G$ must also be bipartite. Assume that $i(G)+k(G)>0$ and that Theorem 4.2.1 holds for graphs with smaller values of $i(G)+k(G)$. Let $D$ be a $\bmod (2 p+1)$-orientation of $G$. As $i(G)+k(G)>0, G$ has a vertex $u$ with

$$
d_{G}(u) \neq 2 p+1 .
$$

Claim 4.1: $G$ has no vertex $v$ with $d_{D}^{+}(v)=d_{D}^{-}(v)$ under the orientation $D$, consequently, $\delta(G) \geq 2 p+1$.

By Lemma 4.1.6(iv), if $d_{G}(v)<2 p+1$ for some $v \in V(G)$, then we must have $d_{D}^{+}(v)=d_{D}^{-}(v)$. Hence it suffices to show that $G$ has no vertex $v$ with $d_{D}^{+}(v)=d_{D}^{-}(v)$.

By contradiction, assume that $G$ has a vertex $v$ with $d_{D}^{+}(v)=d_{D}^{-}(v)=m>0$. Let $v_{1}, v_{2}, \cdots, v_{2 m}$ denote the vertices adjacent to $v$ in $G$ such that $\left(v_{2 l-1}, v\right)$ and $\left(v, v_{2 l}\right)$ are 
in $D$, for $1 \leq l \leq m$. (Note that we allow $v_{i}=v_{j}$ when $i \neq j$. This would happen when $G$ has multiple edges.) For each $l$, let $x_{1}^{l}, x_{2}^{l}, \cdots, x_{2 p+1}^{l}, y_{1}^{l}, y_{2}^{l}, \cdots, y_{2 p+1}^{l}$ be $2(2 p+1)$ new vertices that are not in $V(G)$. Let $K_{2 p, 2 p}(l)-x_{2}^{l} y_{2 p+1}^{l}$ denote the complete bipartite graph with bipartition

$$
\left\{x_{2}^{l}, x_{3}^{l}, \cdots, x_{2 p+1}^{l}\right\} \text { and }\left\{y_{2}^{l}, y_{3}^{l}, \cdots, y_{2 p+1}^{l}\right\}
$$

minus an edge $x_{2}^{l} y_{2 p+1}^{l}$. Let $H\left(x_{1}^{l}, y_{1}^{l}\right)$ denote the graph obtained from $K_{2 p, 2 p}(l)-x_{2}^{l} y_{2 p+1}^{l}$ by adding the vertex $x_{1}^{l}$ that is adjacent to all $x_{2}^{l}, x_{3}^{l}, \cdots, x_{2 p+1}^{l}$ and by adding the new vertex $y_{1}^{l}$ that is adjacent to all $y_{2}^{l}, y_{3}^{l}, \cdots, y_{2 p+1}^{l}$. Obtain a new graph $G_{1}$ from $G-v$ and $H\left(x_{1}^{l}, y_{1}^{l}\right),(1 \leq l \leq m)$, by joining $v_{2 l-1}$ to $x_{1}^{l}$, and $v_{2 l}$ to $y_{1}^{l}$, and $x_{2}^{l+1}$ to $y_{2 p+1}^{l}$, where the superscripts are taken modulo $m$. Orient the edges in $E\left(G_{1}\right)-E(G)$ such that for each $l=1,2, \cdots, m(\bmod m),\left(x_{2}^{l+1}, y_{2 p+1}^{l}\right),\left(v_{2 l-1}, x_{1}^{l}\right),\left(x_{j}^{l}, x_{1}^{l}\right),\left(y_{1}^{l}, v_{2 l}\right),\left(y_{1}^{l}, y_{j}^{l}\right)$, $(2 \leq j \leq 2 p+1)$ are arcs in this orientation of $G_{1}$, and such that all the vertices in $x_{2}^{l}, \cdots, x_{2 p+1}^{l}$ are directed to all the $y_{2}^{l}, \cdots, y_{2 p+1}^{l}$ in $K_{2 p, 2 p}(l)-x_{2}^{l} y_{2 p+1}^{l}$. See Figure 4.1 for an example.
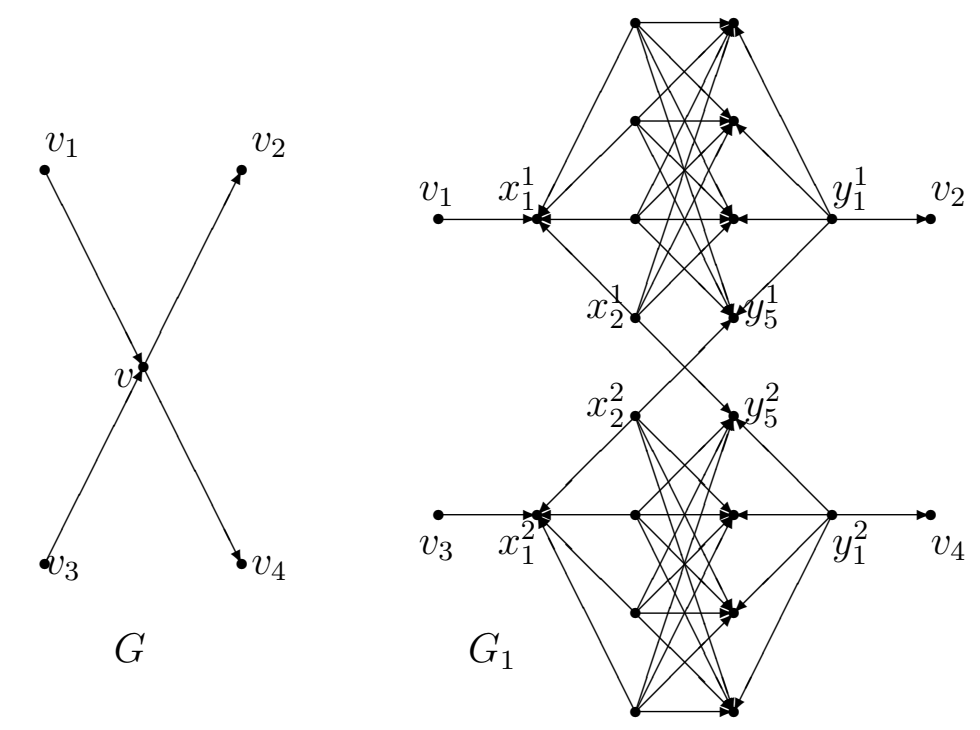

Figure 4.1: Part of the graphs $G$ and $G_{1}$ when $d_{G}(v)=4$ and $p=2($ and so $2 p+1=5)$

Thus the mod $(2 p+1)$-orientation of $E(G)$ together with the orientation on the edges $E\left(G_{1}\right)-E(G)$ is a $\bmod (2 p+1)$-orientation of $G_{1}$. Note that $i\left(G_{1}\right)+k\left(G_{1}\right)=i(G)+$ 
$k(G)-1$, and so by induction, $G_{1}$ is the contraction of a $(2 p+1)$-regular bipartite graph. Since $G$ can be obtained from $G_{1}$ by contracting $\bigcup_{l=1}^{m} H\left(x_{1}^{l}, y_{1}^{l}\right), G$ is also a contraction of a $(2 p+1)$-regular bipartite graph.

Note that the construction and argument above can be applied to any even degree vertex $v$ satisfying $d_{D}^{+}(v)=d_{D}^{-}(v)$ under the orientation $D$. This completes the proof for Claim 4.1.

By Claim 4.1 and by $(4),. d_{G}(u) \geq 2 p+2$, and we may assume that $d_{D}^{+}(u)>d_{D}^{-}(u)$. Since $d_{D}^{+}(u)-d_{D}^{-}(u) \equiv 0(\bmod 2 p+1)$, we must have $d_{D}^{+}(u)>2 p+1$. Let $h=d(u)$ and let $w_{1}, w_{2}, \cdots, w_{h}$ be the vertices adjacent to $u$ in $G$, and assume that $\left(u, w_{i}\right) \in D,(1 \leq i \leq$ $2 p+1)$. (Note that for each $i$ with $h \geq i \geq 2 p+2$, either $\left(u, w_{i}\right)$ or $\left(w_{i}, u\right)$ is an $\operatorname{arc}$ of $D$.) Obtain a new graph $G_{2}$ from $G$ by first splitting $u$ into two vertices $u^{\prime}, u^{\prime \prime}$ such that $u^{\prime}$ is adjacent exactly to $w_{1}, w_{2}, \cdots, w_{2 p}$, and $u^{\prime \prime}$ is adjacent to $w_{2 p+1}, w_{2 p+2}, \cdots, w_{h}$, and then by adding a new edge $e_{u}$ joining $u^{\prime}$ and $u^{\prime \prime}$. Thus we can view that $E\left(G_{2}\right)-\left\{u^{\prime} u^{\prime \prime}\right\}=E(G)$.

Assign an orientation of $G_{2}$ such that the orientation of edges in $E\left(G_{2}\right)-\left\{e_{u}\right\}$ is identical with that in $D$, and such that $\left(u^{\prime}, u^{\prime \prime}\right)$ is an $\operatorname{arc}$ in this orientation of $G_{2}$. See Figure 4.2 for an example.
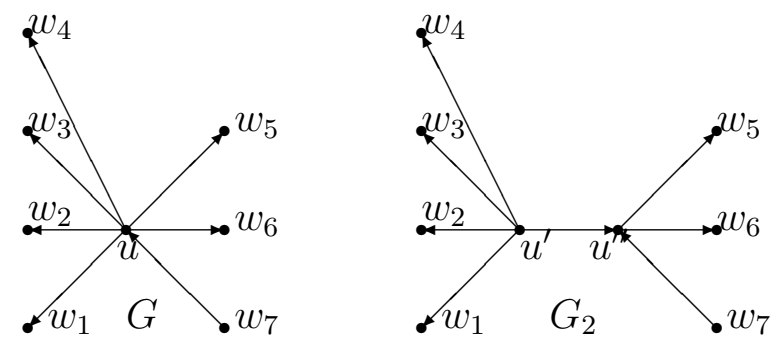

Figure 4.2: Part of the graphs $G$ and $G_{2}$ when $2 p+1=5, d_{D}^{+}(v)=6$ and $d_{D}^{-}(v)=1$

Then the mod (2p+1)-orientation $D$ of $G$ plus the orientation of $e_{u}$ give rise to a mod $(2 p+1)$-orientation of $G_{2}$. As $k\left(G_{2}\right)=k(G)$ and $i\left(G_{2}\right) \leq i(G)-2 p+1$, it follows that $i\left(G_{2}\right)+k\left(G_{2}\right)<i(G)+k(G)$, and so $G_{2}$ is a contraction of a $(2 p+1)$-regular bipartite graph. Since $G=G_{2} / e_{u}, G$ is also a contraction of a $(2 p+1)$-regular bipartite graph. This completes the proof of the theorem. 
CHAPTER 4. CHARACTERIZATION OF GRAPHS WITH MOD (2P+1)-ORIENTATIONS45

Conjecture 4.2.2 Every (4p)-edge-connected graph is the contraction of a $(2 p+1)$-regular bipartite graph. 


\section{Chapter 5}

\section{Characterization of Mod $(2 p+1)$-contractible Graphs}

\subsection{Proof of Theorem 5.1.6}

Mod $(2 p+1)$-contractible graphs defined below play an important role in the investigation of $\bmod (2 p+1)$-orientations. In this section, we shall show that the $\bmod (2 p+1)$ contractible graphs are precisely the graphs in $M_{2 p+1}^{o}$, and present several equivalent ways to describe graphs in $M_{2 p+1}^{o}$.

Definition 5.1.1 Let $p>0$ be an integer. A graph $H$ is $\bmod (2 p+1)$-contractible if for any graph $G$ which contains $H$ as a subgraph,

$$
G \in M_{2 p+1} \text { if and only if } G / H \in M_{2 p+1} \text {. }
$$

By Proposition 1.3.2 and Proposition 1.3.3(C3'), every graph $H \in M_{2 p+1}^{o}$ is $\bmod$ $(2 p+1)$-contractible. It is natural to consider whether every $\bmod (2 p+1)$-contractible graph is in $M_{2 p+1}^{o}$. We first make some observations, stated in the following lemmas. 
Lemma 5.1.2 For any integers $p>0$ and $m, n \geq 0$ with $m \geq n$ and $m-n \equiv 0(\bmod$ $2 p+1)$, there exists a connected bipartite graph $L=L(m, n, 2 p+1)$ with a vertex bipartition $(X, Y)$, such that each of the following holds.

(i) $\forall v \in V(L), d_{L}(v) \in\{1,2 p+1\}$ with $D_{2 p+1}(L) \cap X \neq \varnothing$ and $D_{2 p+1}(L) \cap Y \neq \varnothing$, and (ii) $\left|D_{1}(L) \cap X\right|=m$ and $\left|D_{1}(L) \cap Y\right|=n$.

Proof. First we assume that $0<m=n \leq 2 p$. Let $L(m, m, 2 p+1)$ denote the bipartite graph with vertex bipartition $\left(\left\{x_{0}, x_{1}, x_{2}, \cdots, x_{m}\right\},\left\{y_{0}, y_{1}, y_{2}, \cdots, y_{m}\right\}\right)$, such that

$$
E(L(m, m, 2 p+1))=\left\{x_{0} y_{i}: 1 \leq i \leq m\right\} \cup\left\{y_{0} x_{i}: 1 \leq i \leq m\right\} \cup(2 p+1-m)\left\{x_{0} y_{0}\right\}
$$

where $(2 p+1-m)\left\{x_{0} y_{0}\right\}$ represents a set of $(2 p+1-m)$ parallel edges joining $x_{0}$ and $y_{0}$.

Inductively, assume that we can construct $L(m, m, 2 p+1)$ for smaller values of $m$. For $m=n=2 p+h$, where $h \geq 1$, we by induction can construct a graph $L(h+1, h+$ $1,2 p+1)$ satisfying (i) and (ii) of the lemma, with a vertex bipartition $\left(X^{\prime}, Y^{\prime}\right)$, such that for some $x^{\prime} \in X^{\prime}$ and $y^{\prime} \in Y^{\prime}$ with $x^{\prime}, y^{\prime} \in D_{1}(L(h+1, h+1,2 p+1))$. Then $L(m, m, 2 p+1)$ can be constructed from $L(h+1, h+1,2 p+1)$ by adding new vertices $\left\{x_{1}^{\prime}, x_{2}^{\prime}, \cdots, x_{2 p}^{\prime}\right\},\left\{y_{1}^{\prime}, y_{2}^{\prime}, \cdots, y_{2 p}^{\prime}\right\}$ and by adding new edges $\left\{x^{\prime} y_{i}^{\prime}: 1 \leq i \leq 2 p\right\} \cup\left\{y^{\prime} x_{i}^{\prime}\right.$ : $1 \leq i \leq 2 p\}$. The vertex bipartition of this $L(m, m, 2 p+1)$ is $(X, Y)$ with $X=X^{\prime} \cup$ $\left\{x_{1}^{\prime}, x_{2}^{\prime}, \cdots, x_{2 p}^{\prime}\right\}$ and $Y=Y^{\prime} \cup\left\{y_{1}^{\prime}, y_{2}^{\prime}, \cdots, y_{2 p}^{\prime}\right\}$.

Next, we construct such graphs $L=L(m, n, 2 p+1)$ for the cases when $m-n=$ $k(2 p+1)$, for some $k \geq 0$. We now argue by induction on $k$, and we know that this holds for $k=0$. Suppose that $m-n=k(2 p+1)$ for some $k>0$. Then $m>2 p$. By induction, we assume that we have now constructed such a graph $L(m-2 p, n+1,2 p+1)$ with a vertex bipartition $\left(X^{\prime}, Y^{\prime}\right)$, and for some $y^{\prime} \in Y^{\prime}, y^{\prime} \in D_{1}(L(m-2 p, n+1,2 p+1))$. Obtain $L(m, n, 2 p+1)$ from $L(m-2 p, n+1,2 p+1)$ by adding new vertices $\left\{x_{1}^{\prime}, x_{2}^{\prime}, \cdots, x_{2 p}^{\prime}\right\}$ and by adding new edges $\left\{y^{\prime} x_{i}^{\prime}: 1 \leq i \leq 2 p\right\}$. It is routine to verify that graphs obtained from such constructions do satisfy (i) and (ii) of the lemma, with a vertex bipartition $(X, Y)$ such that $X=X^{\prime} \cup\left\{x_{1}^{\prime}, x_{2}^{\prime}, \cdots, x_{2 p}^{\prime}\right\}$ and $Y=Y^{\prime}$. Hence this lemma is proved by induction. 
Notation 5.1.3 Let $G$ be a connected graph and let $b \in Z\left(G, \mathbf{Z}_{2 p+1}\right)$. Throughout the rest of this section, we assume that $a: V(G) \mapsto\{0, \pm 1, \pm 2, \cdots, \pm 2 p\}$ is a map such that $a_{v}:=a(v) \equiv b(v)(\bmod 2 p+1)$. For each $v \in V(G)$, define

$$
m=\sum_{a_{v}>0} a_{v}, \text { and } n=-\sum_{a_{v}<0} a_{v} .
$$

Since $b \in Z\left(G, \mathbb{Z}_{2 p+1}\right)$, both $m, n \geq 0$ and $m-n=\sum_{v \in V(G)} a_{v} \equiv 0(\bmod 2 p+1)$. Without loss of generality, we may assume that $m \geq n$.

By Lemma 5.1.2, when $m \geq n \geq 0$ with $m-n \equiv 0(\bmod 2 p+1)$, there exists a bipartite graph $L=L(m, n, 2 p+1)$ with a vertex bipartition $(X, Y)$ satisfying Lemma 5.1.2(i) and (ii). Construct a graph $G(b)$ from $G$ and $L$ by identifying $a_{u}$ vertices in $D_{1}(L) \cap X$ with each $u \in V(G)$ with $a_{u}>0$; and by identifying $a_{v}$ vertices in $D_{1}(L) \cap Y$ with each $v \in V(G)$ with $a_{v}<0$.

Lemma 5.1.4 Let $G$ be a connected graph, $b \in Z\left(G, \mathbf{Z}_{2 p+1}\right)$, and $G(b)$ be defined as in Notation 5.1.3. Then $G$ has a $\left(\mathbf{Z}_{2 p+1}, b\right)$-orientation if and only if $G(b)$ has a mod $(2 p+1)$-orientation.

Proof. Suppose that $G(b)$ has a mod $(2 p+1)$-orientation $D$. Let $L=L(m, n, 2 p+1)$ denote the graph in Notation 5.1.3 in the construction of $G(b)$ such that $V(L)$ has a vertex bipartition $(X, Y)$ satisfying Lemma 5.1.2 (i) and (ii). Then $L$ is a subgraph of $G(b)$. By Lemma 4.1.6(i), for each vertex $v \in D_{2 p+1}(L)$, either all edges are directed into $v$, or all directed away from $v$, under this orientation $D$. By replacing the orientation $D$ by $-D$ (the orientation obtained from $D$ by reversing the direction of each edge in $D(G)$ ) if necessary, we may assume that for one $x_{0} \in D_{2 p+1}(L) \cap X$, all edges in $E(G(b))-E(G)$ incident with $x_{0}$ are oriented from $x_{0}$. Since $L$ is a bipartite graph, by the construction of $L$ and by Lemma 4.1.6(i), it follows that $\forall x \in D_{2 p+1}(L) \cap X, d_{D}^{+}(x)=2 p+1$ and $\forall y \in D_{2 p+1} \cap Y, d_{D}^{-}(x)=2 p+1$. Consequently, $\forall v \in V(G)$ with $a_{v}>0$, all edges in $E(G(b))-E(G)$ incident with $v$ are oriented into $v$; and that $\forall v^{\prime} \in V(H)$ with $a_{v^{\prime}}<0$, all edges in $E(G(b))-E(G)$ incident with $v^{\prime}$ are oriented away from $v^{\prime}$. Let $D^{\prime}=D(G)$ denote the restriction of $D(G(b))$ to $E(G)$. Then by the fact that $D$ is a $\bmod (2 p+1)$ orientation of $G(b), \forall v \in V(G), d_{D^{\prime}}^{+}(v)-d_{D^{\prime}}^{-}(v)=a_{v} \equiv b(v)(\bmod 2 p+1)$. Thus the restriction of $D$ to $G$ is a $\left(\mathbb{Z}_{2 p+1}, b\right)$-orientation of $G$. 
Conversely, assume that $G$ has a $\left(\mathbf{Z}_{2 p+1}, b\right)$-orientation $D^{\prime}$. Then $D^{\prime}$ can be extended to an orientation $D$ of $G(b)$ in Notation 5.1 .3 by, for any vertex $v \in V(G)$ with $a_{v}>0$, directing all edges in $E(G(b))-E(G)$ incident with $v$ into $v$, and by for any vertex $w \in V(G)$ with $a_{w}<0$, directing all edges in $E(G(b))-E(G)$ incident with $w$ away from $w$. Then it is routine to verify that $D$ is a $\bmod (2 p+1)$-orientation of $G(b)$.

Our characterization of all mod $(2 p+1)$-contractible graphs will also involve graphs admitting all $(2 p+1)$-orientations, first introduced by Barat and Thomassen in [28].

Definition 5.1.5 ([28]) If for any map $w: V(G) \mapsto \mathbf{Z}_{2 p+1}$ with $\sum_{v \in V(G)} w(v) \equiv|E(G)|$ $(\bmod 2 p+1), G$ has an orientation $D$ such that $\forall v \in V(G), d^{+}(v) \equiv w(v)(\bmod 2 p+1)$, then we say that $G$ admits all $(2 p+1)$-orientations.

Theorem 5.1.6 Let $p \geq 1$ be an integer. The following are equivalent for a connected graph $H$.

(i) $H \in M_{2 p+1}^{o}$,

(ii) $\forall G$ such that $H$ is a subgraph of $G, G / H \in M_{2 p+1}$ if and only if $G \in M_{2 p+1}$.

(iii) $\forall G$ such that $H$ is a subgraph of $G, G / H \in M_{2 p+1}^{o}$ if and only if $G \in M_{2 p+1}^{o}$.

(iv) $H$ admits all $(2 p+1)$-orientations.

Proof. (i) $\Longrightarrow$ (ii). This follows from Proposition 1.3.3 (C3').

(ii) $\Longrightarrow($ i). It suffices to verify by definition that if $H$ is $\bmod (2 p+1)$-contractible, then $H \in M_{2 p+1}^{o}$. Let $b \in Z\left(H, \mathbb{Z}_{2 p+1}\right)$. We adopt Notation 5.1 .3 and obtain $H(b)$ as in Notation 5.1.3. Let $v_{H}$ denote the vertex in $H(b) / H$, and let $L=L(m, n, 2 p+1)$ denote the graph in Notation 5.1.3 in the construction of $H(b)$. Note that $H(b) / H-v_{H} \cong L$ is a bipartite graph, and so $H(b) / H$ satisfies the conditions of Lemma 4.1.6(v). It follows from Lemma $4.1 .6(\mathrm{v})$ that $H(b) / H$ has a $\bmod (2 p+1)$-orientation. Since $H$ is $\bmod$ $(2 p+1)$-contractible, it then follows from Definition 5.1.1 that $H(b)$ has a mod $(2 p+1)$ orientation D. By Lemma 5.1.4, $H$ has a $\left(\mathbf{Z}_{2 p+1}, b\right)$-orientation. As $b$ is arbitrary, by definition, $H \in M_{2 p+1}^{o}$.

(i) $\Longleftrightarrow$ (iii). This follows from Proposition 1.3.3 (C2) and (C3). 
(iv) $\Longrightarrow$ (i). Suppose that $G$ admits all generalized $(2 p+1)$-orientations. We are to show that $G \in M_{2 p+1}^{o}$. Let $b \in Z\left(G, \mathbb{Z}_{2 p+1}\right)$. Define $w: V(G) \mapsto \mathbb{Z}_{2 p+1}$ be given by $w(v) \equiv(p+1)\left(d_{G}(v)+b(v)\right),(\bmod 2 p+1), \forall v \in V(G)$. Then

$$
\begin{aligned}
\sum_{v \in V(G)} w(v) & \equiv \sum_{v \in V(G)}(p+1)\left(d_{G}(v)+b(v)\right) \\
& \equiv \sum_{v \in V(G)}(p+1) d_{G}(v) \equiv 2(p+1)|E(G)| \equiv|E(G)|(\bmod 2 p+1) .
\end{aligned}
$$

Since $G$ admits all generalized $(2 p+1)$-orientations, $G$ has an orientation $D$ such that $w(v) \equiv d_{D}^{+}(v)(\bmod 2 p+1), \forall v \in V(G)$. Let $f(e) \equiv 1, \forall e \in E(G)$. As $2(p+1) \equiv 1(\bmod$ $2 p+1), \forall v \in V(G), \partial f(v)=d^{+}(v)-d^{-}(v) \equiv w(v)-d^{-}(v) \equiv(p+1)\left(d_{G}(v)+b(v)\right)-d^{-}(v) \equiv$ $(p+1)\left(d^{+}(v)+d^{-}(v)+b(v)\right)-d^{-}(v) \equiv \frac{1}{2}\left(d^{+}(v)-d^{-}(v)+b(v)\right)(\bmod 2 p+1)$. Thus $\partial f(v)=d^{+}(v)-d^{-}(v) \equiv b(v),(\bmod 2 p+1)$. By definition, $G \in M_{2 p+1}^{o}$.

(i) $\Longrightarrow$ (iv). Now we assume that $G \in M_{2 p+1}^{o}$. Let $w: V(G) \mapsto \mathbb{Z}_{2 p+1}$ be such that $\sum_{v \in V(G)} w(v) \equiv|E(G)|(\bmod 2 p+1)$. Define $b: V(G) \mapsto \mathbf{Z}_{2 p+1}$ be given by

$$
b(v) \equiv 2 w(v)-d_{G}(v)(\bmod 2 p+1) .
$$

Then $\sum_{v \in V(G)} b(v) \equiv \sum_{v \in V(G)} 2 w(v)-\sum_{v \in V(G)} d_{G}(v) \equiv 2|E(G)|-2|E(G)| \equiv 0(\bmod$ $2 p+1)$, and so $b \in Z\left(G, \mathbf{Z}_{2 p+1}\right)$. Since $G \in M_{2 p+1}^{o}, G$ has an orientation $D$, such that when $f \equiv 1, \partial f \equiv b$. Thus at each vertex $v \in V(G), d^{+}(v)-d^{-}(v)=\partial f(v) \equiv b(v) \equiv$ $2 w(v)-d_{G}(v) \equiv 2 w(v)-d^{+}(v)-d^{-}(v)(\bmod 2 p+1)$. It follows that $2 d^{+}(v) \equiv 2 w(v)$ $(\bmod 2 p+1)$, and so $d^{+}(v) \equiv w(v)(\bmod 2 p+1)$, as desired. 


\section{Chapter 6}

\section{Some Families of Graphs That Are Mod $(2 p+1)$-contractible}

\subsection{Graphs without $K_{4}$-minors}

A subgraph $H$ of a graph $G$ is a $K_{4}$-minor if $H$ can be contracted onto a $K_{4}$. In this section, we shall show a sharp lower bound of edge-connectivity for a $K_{4}$-minor free graph to be in $M_{2 p+1}^{o}$. We need a former theorem from Dirac.

Theorem 6.1.1 (Dirac [31]) If $G$ is a simple graph without a $K_{4}$-minor, then $G$ has a vertex of degree at most 2 .

Corollary 6.1.2 Every $(4 p-1)$-edge-connected graph without a $K_{4}$-minor is in $M_{2 p+1}^{o}$.

Proof. Let $G$ be a ( $4 p-1)$-edge-connected graph without a $K_{4}$-minor, and let $G_{0}$ denote the simplification of $G$. The conclusion holds trivially if $|V(G)|=1$. We assume that $|V(G)|>1$ and the conclusion of the corollary holds also for graphs with smaller order.

Since $G$ has no $K_{4}$-minor, $G_{0}$ does not have a $K_{4}$-minor either. By Dirac's Theorem, $G_{0}$ must have a vertex $w$ of degree 1 or 2 . If $w$ has degree 1 and is incident with the 
only edge $e$ in $G_{0}$, then since $\kappa^{\prime}(G) \geq 4 p-1, G$ must have a subgraph $H$ isomorphic to $(4 p-1) K_{2}$. If $w$ has degree 2 and is incident with the edges $e_{1}$ and $e_{2}$ in $G_{0}$, then since $\kappa^{\prime}(G) \geq 4 p-1$, one of $e_{1}$ and $e_{2}$ must be in a set of at least $2 p$ parallel edges, and so $G$ must have a subgraph $H$ isomorphic to $(2 p) K_{2}$. By Lemma 4.1.1(ii), $H \in M_{2 p+1}^{o}$. Since $G$ has no $K_{4}$-minors, $G / H$ also has no $K_{4}$-minors. Moreover, $\kappa^{\prime}(G / H) \geq \kappa^{\prime}(G)$. It follows by induction that $G / H \in M_{2 p+1}^{o}$. Since $H \in M_{2 p+1}^{o}$ and by (C3) of Proposition 1.3.3, $G \in M_{2 p+1}^{o}$, and so the corollary is proved by induction.

The next example indicates that the edge-connectivity condition cannot be relaxed.

Example 6.1.3 Let $m=2 p-1$ and let $G=m C_{2 p+1}$. Choose the constant function $b \in$ $Z\left(G, \mathbb{Z}_{2 p+1}\right)$ such that $\forall v \in V(G), b(v)=1$. Assume that $G$ has a $\left(\mathbb{Z}_{2 p+1}, b\right)$-orientation $D$. Then for any vertex $v \in V(G)$, we have

$$
\left\{\begin{array}{l}
d^{+}(v)+d^{-}(v)=4 p-2 \\
d^{+}(v)-d^{-}(v) \equiv 1(\bmod 2 p+1)
\end{array}\right.
$$

It follows that either $d^{+}(v)=3 p$ and $d^{-}(v)=p-2$ (referred as a positive vertex) or $d^{-}(v)=3 p-1$ and $d^{+}(v)=p-1$ (referred as a negative vertex). It follows that no two positive vertices are adjacent, and no two negative vertices are adjacent. This implies that $G$ must be bipartite, contrary to the fact that $G$ has an odd circuit of length $2 p+1$. Hence $G$ does not have a $\left(\mathbb{Z}_{2 p+1}, b\right)$-orientation, and so $G \notin M_{2 p+1}^{o}$.

\subsection{Chordal Graphs}

Throughout this section, $p$ denotes a positive integer, and a graph $H \in M_{2 p+1}^{o}$ will be refereed as an $M_{2 p+1}^{o}$-graph. A simple graph $G$ is chordal if every cycle of length greater than 3 possesses a chord. Equivalently speaking, a simple graph $G$ is chordal if every induced cycle of $G$ has length 3. In Theorem 4.2 of [36], it has been proved that every 4-connected chordal graph is in $M_{3}^{o}$. The purpose of this section is to extend this Theorem 4.2 of [36] to the main result of this section below.

Theorem 6.2.1 Every simple $(4 p)$-connected chordal graph is in $M_{2 p+1}^{o}$. 
To prove this theorem, we need some lemmas.

Lemma 6.2.2 (Lemma 2.1.2 of [35]) A graph $G$ is chordal if and only if every minimal vertex cut induces a complete subgraph of $G$.

Lemma 6.2.3 Let $T$ be a connected spanning subgraph of $G$. If for each edge e $\in E(T)$, $G$ has a subgraph $H_{e} \in M_{2 p+1}^{o}$ with $e \in E\left(H_{e}\right)$, then $G \in M_{2 p+1}^{o}$.

Proof. We argue by induction on $|V(G)|$. The lemma holds trivially if $|V(G)|=1$. Assume that $|V(G)|>1$ and pick an edge $e^{\prime} \in E(T)$. Then $G$ has a subgraph $H^{\prime} \in M_{2 p+1}^{o}$ such that $e^{\prime} \in E\left(H^{\prime}\right)$. Let $G^{\prime}=G / H^{\prime}$ and let $T^{\prime}=T /\left(E\left(H^{\prime}\right) \cap E(T)\right)$. Since $T$ is a connected spanning subgraph of $G, T^{\prime}$ is a connected spanning subgraph of $G^{\prime}$. For each $e \in E\left(T^{\prime}\right), e \in E(T)$, and so by assumption, $G$ has a subgraph $H_{e} \in M_{2 p+1}^{o}$ with $e \in E\left(H_{e}\right)$. By Proposition 1.3.3(C2), $H_{e}^{\prime}=H_{e} /\left(E\left(H_{e}\right) \cap E\left(H^{\prime}\right)\right) \in M_{2 p+1}^{o}$ and $e \in H_{e}^{\prime}$. Therefore by induction $G^{\prime} \in M_{2 p+1}^{o}$. Then by Proposition 1.3.3(C3), and by the assumption that $H^{\prime} \in M_{2 p+1}^{o}, G \in M_{2 p+1}^{o}$.

Proof of Theorem 6.1: Let $G$ be a $(4 p)$-connected chordal graph. If $G$ itself is a clique, then as $\kappa(G) \geq 4 p, G \cong K_{m}$ for some integer $m \geq 4 p+1$, and so by Proposition 4.1.3(v), $G \in M_{2 p+1}^{o}$. Thus throughout the rest of the proof, we assume that $G$ is not a complete graph.

By Lemma 6.2.3, it suffices to show that every edge $e \in E(G)$ lies in a subgraph $H_{e}$ of $G$ with $H_{e} \in M_{2 p+1}^{o}$. Let $e=x y$ be an edge in $G$. For any vertex $v \in V(G)$, let $N(v)$ denote the vertices adjacent to $v$ in $G$. We shall show in each of the following two cases concerning the possibilities of $e$, a subgraph $H_{e} \in M_{2 p+1}^{o}$ can always be found such that $e \in E\left(H_{e}\right)$.

Case 1: $N(x) \neq V(G)-\{x\}$ or $N(y) \neq V(G)-\{y\}$.

Without loss of generality, we assume that $N(x) \neq V(G)-\{x\}$. Then $G$ has a vertex $z$ such that $x z \notin E(G)$. Since $G$ is 2-connected and not a complete graph, $N(x)$ contains a minimal vertex cut $X$ of $G$ which separates $x$ and $z$. By Lemma 6.2.2, $G[X]$ is a complete graph. Since $x$ is adjacent to every vertex in $N(x), G[X \cup\{x\}] \cong K_{m_{x}}$ is a 
complete subgraph of $G$ with order $m_{x}=|X|+1 \geq \kappa(G)+1=4 p+1$. It follows that $m_{x} \geq 4 p+1$ and so by Proposition 4.1.3(v), $G[X \cup\{x\}] \in M_{2 p+1}^{o}$. If $y \in X$, then we define $H_{e}=G[X \cup\{x\}] \in M_{2 p+1}^{o}$.

Hence we assume that $y \notin X$ for any minimal vertex cut $X \subseteq N(x)$. It follows that $N(y) \subseteq N(x) \cup\{x\}$. Otherwise, if there exists $t \in V(G)-(N(x) \cup\{x\})$ such that $y z \in E(G)$, then there is a minimal vertex cut of $N(x)$ containing $y$ which separates $x$ and $t$, a contradiction. Since $N(y) \subseteq N(x) \cup\{x\}, y z \notin E(G)$, and so $N(y)$ contains a minimal vertex cut $Y$. By Lemma 6.2.2 and by the assumption of $\kappa(G) \geq 4 p, G[Y \cup\{y\}]$ is a complete graph of order at least $4 p+1$, and so by Proposition 4.1.3(v), $G[Y \cup\{y\}] \in M_{2 p+1}^{o}$.

If $x \in Y$, then we define $H_{e}=G[Y \cup\{y\}] \in M_{2 p+1}^{o}$. Hence we assume further that $x \notin Y$ for any minimal vertex cut $Y \subseteq N(y)$, and so $x$ and $y$ must be in the same component of $G-Y$. For a such fix $Y$, by Lemma 6.2.2 and the assumption of $\kappa(G) \geq 4 p$, $G[Y]$ is a complete subgraph of $G$ with order at least $4 p$. Note that $Y \subseteq N(y) \subseteq N(x) \cup\{x\}$ and $x \notin Y$. It follows that $G[Y \cup\{x, y\}]$ is a complete subgraph of $G$ with order at least $4 p+2$, and so by Proposition 4.1.3(v), $G[Y \cup\{x, y\}] \in M_{2 p+1}^{o}$. Therefore in this final subcase of Case 1 , we define $H_{e}=G[Y \cup\{x, y\}]$.

Case 2: Both $N(x)=V(G)-\{x\}$ and $N(y)=V(G)-\{y\}$.

Since $G$ is not a complete graph itself, $G$ has vertices $v, v^{\prime} \in V(G)-\{x, y\}$ such that $v v^{\prime} \notin E(G)$. Therefore, $N(v)$ contains a minimal vertex cut $X^{\prime}$ separating $v$ and $v^{\prime}$ in $G$. By Lemma 6.2.2 and by the assumption of $\kappa(G) \geq 4 p, W=G\left[X^{\prime} \cup\{v\}\right]$ is a complete graph of order at least $4 p+1$, and so by Proposition 4.1.3(v), $W \in M_{2 p+1}^{o}$. Since both $N(x)=V(G)-\{x\}$ and $N(y)=V(G)-\{y\}$, both $x$ and $y$ must be in $X^{\prime}$, and so $e=x y \in W$. It is now natural to define $H_{e}=W$.

Since in either case, we can always find a subgraph $H_{e} \in M_{2 p+1}^{o}$ such that $e \in E\left(H_{e}\right)$, it follows by Lemma 6.2.3 that $G \in M_{2 p+1}^{o}$.

Definition 6.2.4 (Definition 2.1.8 of [35]) Let $k>0$ be an integer. A clique with order $k+1$ is a $k$-tree; given a $k$-tree $T_{n}$ on $n$ vertices, a $k$-tree with $n+1$ vertices is constructed by taking $T_{n}$ and creating a new vertex $x_{n+1}$ which is made adjacent to a $k$-clique of $T_{n}$, 
and non-adjacent to any of the other $n-k$ vertices of $T_{n}$.

Corollary 6.2.5 Every $k$-tree with $k \geq 4 p$ is in $M_{4 p+1}^{o}$.

Proof. We may assume that $G$ is a $k$-tree but not a clique. By Lemma 6.2.2, every $k$-tree is also a chordal graph. By the definition of a $k$-tree, it is routine to verify that $\kappa(G) \geq k$. It now follows by Theorem 6.2.1 that, if $k \geq 4 p$, every $k$-tree must be in $M_{2 p+1}^{o}$.

By Example 4.1.5, the complete graph $K_{4 p}$ is a $(4 p-1)$-tree which is not in $M_{2 p+1}^{o}$. This shows that Corollary 6.2 .5 is best possible. 


\section{Chapter 7}

\section{Mod $(2 p+1)$-orientation of Line Graphs}

\section{$7.1 \quad$ Introduction}

In [41], the following theorem is proved.

Theorem 7.1.1 (Chen et al, Theorem 1.4 in [41]) If $G$ has a 3-NZF and the minimum degree of $G$ is at least 4 , then $L(G)$ also has a $3-N Z F$.

In this chapter, we prove that the Conjecture 1.3.1 (ii) and (iii) on line graphs would imply the truth of the conjectures in general.

Theorem 7.1.2 Let $G$ be a graph and $k$ be an integer. Then the following statements are equivalent.

(i) If $\kappa^{\prime}(G) \geq k$, then $G \in M_{2 p+1}$.

(ii) If $\kappa^{\prime}(L(G)) \geq k$, then $L(G) \in M_{2 p+1}$. 
Theorem 7.1.3 Let $G$ be a graph and $k$ be an integer. Then the following statements are equivalent.

(i) If $\kappa^{\prime}(G) \geq k$, then $G \in M_{2 p+1}^{o}$.

(ii) If $\kappa^{\prime}(L(G)) \geq k$, then $L(G) \in M_{2 p+1}^{o}$.

We also extend Theorem 7.1.1 to the following.

Theorem 7.1.4 Let $G$ be a graph. If $G \in M_{2 p+1}$ and $\delta(G) \geq 4 p$, then $L(G) \in M_{2 p+1}$.

\subsection{Preliminaries}

In this section, we review some useful results needed in the arguments.

Lemma 7.2.1 (Proposition 4.1.3(v) and Example 4.1.5) A complete graph $K_{m} \in M_{2 p+1}^{o}$ if and only if $m=1$ or $m \geq 4 p+1$.

The following lemma follows from the definition of line graph.

Lemma 7.2.2 Let $G$ be a graph with $E(G) \neq \varnothing$ and let $e \in E(G)$ such that the two ends of $e$ are $u$ and $v$. Let $G(e)$ be the graph obtained from $G$ by replacing e by $a(u, v)$-path $u v_{e} v$ of length 2. Let $e^{\prime}$ denote the edge in $L(G(e))$ that has $u v_{e}$ and $v_{e} v$ as its ends. Then

$$
L(G(e)) /\left\{e^{\prime}\right\}=L(G)
$$

Let $G$ be a graph and let $S(G)$, the subdivided graph of $G$, be the graph obtained from $G$ by replacing each edge $e$ of $G$ by a path of length 2 with a newly added internal vertex $v_{e}$. Note that the correspondence $e \leftrightarrow e^{\prime}$ defined in Lemma 7.2.2 is a bijection between $E(G)$ and $\left\{e^{\prime} \mid e \in E(G)\right\} \subset E(L(S(G)))$. Define

$$
E^{\prime}(G)=\left\{e^{\prime} \in E(L(S(G))) \mid e \in E(G)\right\}
$$


Then clearly,

$$
L(G)=L(S(G)) / E^{\prime}(G)
$$

and

$$
E(L(S(G)))-E^{\prime}(G)=\cup_{v \in V(G)} E\left(L\left(E_{S(G)}(v)\right)\right),
$$

so we have

$$
L(S(G)) /\left[E(L(S(G)))-E^{\prime}(G)\right]=G .
$$

Example: Let $G$ be the graph shown in Fig 1. And $L(G), S(G)$ and $L(S(G))$ are also shown here. Note that $E^{\prime}(G)=\left\{e_{i}^{\prime}: 1 \leq i \leq 5\right\}$. It's easy to check that Equations (7.1), (7.2) and (7.3) hold from the graphs.

\subsection{Main results}

Lemma 7.3.1 Let $T$ be a connected spanning subgraph of $G$. If for each edge e $\in E(T)$, $G$ has a subgraph $H_{e} \in M_{2 p+1}^{o}$ with $e \in E\left(H_{e}\right)$, then $G \in M_{2 p+1}^{o}$.

Proof. We argue by induction on $|V(G)|$. The lemma holds trivially if $|V(G)|=1$. Assume that $|V(G)|>1$ and pick an edge $e^{\prime} \in E(T)$. Then $G$ has a subgraph $H^{\prime} \in M_{2 p+1}^{o}$ such that $e^{\prime} \in E\left(H^{\prime}\right)$. Let $G^{\prime}=G / H^{\prime}$ and let $T^{\prime}=T /\left(E\left(H^{\prime}\right) \cap E(T)\right)$. Since $T$ is a connected spanning subgraph of $G, T^{\prime}$ is a connected spanning subgraph of $G^{\prime}$. For each $e \in E\left(T^{\prime}\right), e \in E(T)$, and so by assumption, $G$ has a subgraph $H_{e} \in M_{2 p+1}^{o}$ with $e \in E\left(H_{e}\right)$. By Proposition 1.3.3(C2), $H_{e}^{\prime}=H_{e} /\left(E\left(H_{e}\right) \cap E\left(H^{\prime}\right)\right) \in M_{2 p+1}^{o}$ and $e \in H_{e}^{\prime}$. Therefore by induction $G^{\prime} \in M_{2 p+1}^{o}$. Then by Proposition 1.3.3(C3), and by the assumption that $H^{\prime} \in M_{2 p+1}^{o}, G \in M_{2 p+1}^{o}$.

Lemma 7.3.2 Let $G$ be a graph. If $\delta(G) \geq 4 p+1$, then $L(G) \in M_{2 p+1}^{o}$.

Proof. Since $\delta(G) \geq 4 p+1$, for any $e \in L(G), e \in K_{m}$ with $m \geq 4 p+1$. By Lemma 7.2.1, $K_{m} \in M_{2 p+1}^{o}$. Therefore, $L(G) \in M_{2 p+1}^{o}$ by Lemma 7.3.1. 
Lemma 7.3.3 Let $G$ be a graph and $k$ be an integer. If $\kappa^{\prime}(G) \geq k$, then

$$
\kappa^{\prime}(L(S(G))) \geq k
$$

Proof. By contradiction, suppose $X$ is an edge cut of $L(S(G))$ satisfies: (1) $|X|<k$ and $|X|$ is minimized; and (2) $\left|X \cap E^{\prime}(G)\right|$ is maximized subject to (1). Since $\kappa^{\prime}(G) \geq k$, $\delta(G) \geq k$. Note that for any $x \in V(L(S(G))), x \in K_{m}$ with $m \geq k$ and $\left|E_{x}\right|=1$ where $E_{x}=\left\{e=x y \in L(S(G)) \mid y \notin V\left(K_{m}\right)\right\}$. Therefore, $\delta(L(S(G))) \geq k$.

If $X \subseteq E^{\prime}(G)$, by Equation (7.3), $X$ is also an edge cut of $G$. Therefore, $|X| \geq k$, contrary to $|X|<k$.

Suppose there exists $e=u v \in X-E^{\prime}(G)$, then $e$ is in some $K_{m}$ and is adjacent to some $e^{\prime}=u v^{\prime}$ with $v^{\prime} \notin V\left(K_{m}\right)$. Let $H$ be one of the components of $L(S(G))-X$. If $H$ contains only one vertex, then $|X| \geq \delta(L(S(G))) \geq k$. If $H$ contains at least 2 vertices, let

$$
X^{\prime}=\left(X-E\left(K_{m}\right)\right) \cup\left\{u_{i} v_{i} \in E^{\prime}(G): u_{i} \in K_{m} \cap H, v_{i} \notin K_{m}\right\}
$$

then $\left|X^{\prime}\right| \leq|X|$ and $\left|X^{\prime} \cap E^{\prime}(G)\right|>\left|X \cap E^{\prime}(G)\right|$ and $X^{\prime}$ is also an edge cut of $L(S(G))$, contrary to that $|X|$ is minimized and $\left|X \cap E^{\prime}(G)\right|$ is maximized.

Hence, $\kappa^{\prime}(L(S(G))) \geq k$.

Proof of Theorem 7.1.2 (i) $\Rightarrow$ (ii) It is trivial.

(ii) $\Rightarrow$ (i) Since $\kappa^{\prime}(G) \geq k, \kappa^{\prime}(L(S(G))) \geq k$ by Lemma 7.3.3. Then by the assumption of part (ii), $L(S(G)) \in M_{2 p+1}$. Note that $G$ is a contraction of $L(S(G)$ ) by Equation (7.3). Thus $G \in M_{2 p+1}$ by Proposition 1.3.3 (C2'). $\square$

Corollary 7.3.4 To prove Conjecture 1.3.1 (ii), it suffices to prove that if $\kappa^{\prime}(L(G)) \geq 4 p$, then $L(G) \in M_{2 p+1}$, for any graph $G$.

The proof of Theorem 7.1.3 is similar to that of Theorem 7.1.2. 
Proof of Theorem 7.1.3 (i) $\Rightarrow$ (ii) It is trivial.

(ii) $\Rightarrow$ (i) Since $\kappa^{\prime}(G) \geq k, \kappa^{\prime}(L(S(G))) \geq k$ by Lemma 7.3.3. Then by the assumption of part (ii), $L(S(G)) \in M_{2 p+1}^{0}$. Note that $G$ is a contraction of $L(S(G))$ by Equation (7.3). Thus $G \in M_{2 p+1}^{0}$ by Proposition 1.3.3 (C2). $\square$

Corollary 7.3.5 To prove Conjecture 1.3.1 (iii), it suffices to prove that if $\kappa^{\prime}(L(G)) \geq$ $4 p+1$, then $L(G) \in M_{2 p+1}^{o}$, for any graph $G$.

Lemma 7.3.6 If $G \in M_{2 p+1}$ and $\delta(G)=4 p$, then $L(G) \in M_{2 p+1}$.

Proof. By Proposition 1.3.3(C2') and Equation (7.1), it suffices to prove that $L(S(G)) \in$ $M_{2 p+1}$.

Since $G \in M_{2 p+1}, G$ has an orientation $D$ such that at every vertex $v \in V(G)$,

$$
d_{D}^{+}(v)-d_{D}^{-}(v) \equiv 0(\bmod 2 p+1) .
$$

Note that by Equation (7.3), $D$ is an orientation of a subgraph of $L(S(G))$. By Equation (7.2), $E(L(S(G)))-E^{\prime}(G)$ is a disjoint union of $K_{d(v)}$ with $v \in V(G)$. By Equations (7.2) and (7.4), under the orientation $D$,

$$
d_{D}^{+}\left(K_{d(v)}, L(S(G))-K_{d(v)}\right)-d_{D}^{-}\left(K_{d(v)}, L(S(G))-K_{d(v)}\right) \equiv 0(\bmod 2 p+1),
$$

and for any vertex $u \in K_{d(v)}$

$$
\left|E_{G}\left(u, L(S(G))-K_{d(v)}\right)\right|=1 .
$$

If $d(v) \geq 4 p+1$, then by Lemma 7.2.1, $K_{d(v)} \in M_{2 p+1}^{o}$, and so there exists an orientation $D_{v}$ of $K_{d(v)}$ such that $d_{D_{v}}^{+}(u)-d_{D_{v}}^{-}(u) \equiv 0(\bmod 2 p+1)$ in $L(S(G))$ at every vertex $u \in K_{d(v)}$.

Now suppose $d(v)=4 p$ and let $H=K_{d(v)}=K_{4 p}$. By Equations (7.5) and (7.6), there exists partition $(U, V)$ of $H$ where $U=\left\{u_{1}, u_{2}, \cdots, u_{2 p}\right\}$ and $V=\left\{v_{1}, v_{2}, \cdots, v_{2 p}\right\}$, such that under the orientation $D$,

$$
d_{D}^{+}\left(u_{i}, L(S(G))-H\right)-d_{D}^{-}\left(u_{i}, L(S(G))-H\right)=1
$$


and

$$
d_{D}^{+}\left(v_{i}, L(S(G))-H\right)-d_{D}^{-}\left(v_{i}, L(S(G))-H\right)=-1 .
$$

Let $M(v)=\left\{u_{i} v_{i} \mid u_{i} \in U, v_{i} \in V\right\}$ be a perfect matching of $H=K_{d(v)}$. Then $H-M(v)$ is a $(4 p-2)$-regular graph, and so $H-M(v)$ is Eulerian. Therefore, $H-M(v)$ has an orientation $D_{M(v)}$ such that for any $x \in V(H), d_{D_{M(v)}}^{+}(x)-d_{D_{M(v)}}^{-}(x)=0(\bmod 2 p+1)$ in $H-M(v)$. Then we define an orientation $D_{M(v)}^{\prime}$ for $M(v)$ as $h e a d\left(u_{i} v_{i}\right)=u_{i}$ and $\operatorname{tail}\left(u_{i} v_{i}\right)=v_{i}$. Let $D_{v}$ be the disjoint union of $D_{M(v)}$ and $D_{M(v)}^{\prime}$.

Thus the disjoint union of $D$ and all $D_{v}$ with $v \in V(G)$ gives an orientation $D^{\prime}$ of $L(S(G))$. It is routine to verify that $d_{D^{\prime}}^{+}(x)-d_{D^{\prime}}^{-}(x) \equiv 0(\bmod 2 p+1)$ at every vertex $x \in V(L(S(G)))$.

Hence, $L(S(G)) \in M_{2 p+1}$.

Theorem 7.1.4 now follows from Lemmas 7.3.6 and 7.3.2. When $p=1$, we obtain Theorem 7.1.1, restated as the following corollary.

Corollary 7.3.7 If $G \in M_{3}$ and $\delta(G) \geq 4$, then $L(G) \in M_{3}$. 


\section{Chapter 8}

\section{Graphs with Disjoint Spanning Trees}

\subsection{Introduction}

We consider the problem of designing networks with $n$ processors $v_{1}, v_{2}, \cdots, v_{n}$ such that, for a given sequence of positive integers $d_{1}, d_{2}, \cdots, d_{n}$, it is expected that each processor $v_{i}$ will be connected to other processors by $d_{i}$ connections. It is further expected that such networks will have certain level of strengths. This problem can be modelled as the problem of determining whether a (graphical) degree sequence has realizations with certain graphical properties. Motivated by the research in [44], we shall consider the strength of the graph as the property of having $k$ edge-spanning trees.

This chapter studies finite and undirected graphs without loops. Undefined terms can be found in [1]. In particular, $\omega(G)$ denotes the number of components of a graph $G$. For a vertex $v \in V(G)$ and a subgraph $K$ of $G, d_{K}(v)$ is the number of vertices in $K$ that are adjacent to $v$ in $G$. If $X \subseteq E(G)$, then $G[X]$ is the subgraph of $G$ induced by the edge subset $X$, and $G(X)$ is the spanning subgraph of $G$ with edge set $X$. A sequence $d=\left(d_{1}, d_{2}, \cdots, d_{n}\right)$ is nonincreasing if $d_{1} \geq d_{2} \geq \cdots \geq d_{n}$. A sequence $d=\left(d_{1}, d_{2}, \cdots, d_{n}\right)$ is graphic if there is a simple graph $G$ with degree sequence $d$. In this case, this graph $G$ is a realization of $d$. We will also call $G$ a d-realization. 
Many researchers have been investigating graphic degree sequences that have a realization with certain graphical properties. See [43], [45], [46], [47], [52], [53], and [54], among others. An excellent and resourceful survey by J. Li can be found in [50].

In this chapter, we focus on the investigation of graphic sequences that have realizations with many edge-disjoint spanning trees.

In Section 8.2, we develop some useful properties related to graphs with at least $k$ edgedisjoint spanning trees. In Section 8.3, we present a proof for the following characterization of graphic sequences with realizations having $k$ edge-disjoint spanning trees.

Theorem 8.1.1 A nonincreasing graphic sequence $d=\left(d_{1}, d_{2}, \cdots, d_{n}\right)$ has a realization $G$ with $k$ edge-disjoint spanning trees if and only if either $n=1$ and $d_{1}=0$, or $n \geq 2$ and both of the following hold:

(i) $d_{n} \geq k$.

(ii) $\sum_{i=1}^{n} d_{i} \geq 2 k(n-1)$.

\subsection{Properties of Graphs with $k$ edge-disjoint Span- ning Trees}

Let $G$ be a graph, and $k \geq 2$ be an integer. Let $\tau(G)$ denote the number of edge-disjoint spanning trees of $G$, and $\mathcal{T}_{k}$ the set of all graphs with $\tau(G) \geq k$. By definition, $K_{1} \in \mathcal{T}_{k}$, for any integer $k>0$. In this section, we summarize and develop some useful properties on $\mathcal{T}_{k}$, some of which were first introduced in [51], and are later extended to matroids in [48] and [49].

Proposition 8.2.1 (Liu et al, Lemma 2.1 in [51]) For any integer $k, \mathcal{T}_{k}$ is a family of connected graphs such that each of the following holds.

(C1) $K_{1} \in \mathcal{T}_{k}$.

(C2) If $e \in E(G)$ and if $G \in \mathcal{T}_{k}$, then $G / e \in \mathcal{T}_{k}$.

(C3) If $H$ is a subgraph of $G$, and if $H, G / H \in \mathcal{T}_{k}$, then $G \in \mathcal{T}_{k}$. 
(C4) If $H_{1}$ and $H_{2}$ are two subgraphs of $G$ such that $H_{1}, H_{2} \in \mathcal{T}_{k}$ and $V\left(H_{1}\right) \cap V\left(H_{2}\right) \neq \varnothing$, then $H_{1} \cup H_{2} \in \mathcal{T}_{k}$.

Define the density of a subgraph $H$ of $G$ with $|V(H)|>1$ as follows:

$$
d(H)=\frac{|E(H)|}{|V(H)|-1}, \quad \text { if }|V(H)|>1 .
$$

Theorem 8.2.2 (Yao et al., Theorem 2.4 in [55]) Let $G$ be a multigraph. If $d(G) \geq k$, then $G$ has a nontrivial subgraph $H$ such that $H \in \mathcal{T}_{k}$.

Let $G$ be a nontrivial connected graph. For any positive integer $r$, a nontrivial subgraph $H$ of $G$ is $\mathcal{T}_{r}$-maximal if both $H \in \mathcal{T}_{r}$ and $H$ has no proper subgraph $K$ of $G$, such that $K \in \mathcal{T}_{r}$. A $\mathcal{T}_{r}$-maximal subgraph $H$ of $G$ is called an $r$-region if $r=\tau(H)$. Define $\bar{\tau}(G)=\max \{r: G$ has a subgraph as an $r$-region $\}$.

Lemma 8.2.3 (Liu et al, Lemma 2.3 in [51]) Let $r, r^{\prime}>0$ be integers, $H, H^{\prime}$ be an $r$-region and an $r^{\prime}$-region of $G$, respectively. Then exactly one of the following must hold:

(i) $V(H) \cap V\left(H^{\prime}\right)=\varnothing$,

(ii) $r^{\prime}=r$ and $H=H^{\prime}$,

(iii) $r^{\prime}>r$ and $H$ is a nonspanning subgraph of $H^{\prime}$,

(iv) $r^{\prime}<r$ and $H$ contains $H^{\prime}$ as a non-spanning subgraph.

Theorem 8.2.4 (Theorem 2.4 in [51]) Let $G$ be a nontrivial connected graph. Then (a) there exists a positive integer $m$, and an $m$-tuple $\left(i_{1}, i_{2}, \cdots, i_{m}\right)$ of positive integers with

$$
\tau(G)=i_{1}<i_{2}<\cdots<i_{m}=\bar{\tau}(G)
$$

and a sequence of edge subsets

$$
E_{m} \subset \cdots \subset E_{2} \subset E_{1}=E(G)
$$

such that each component of the induced subgraphs $G\left[E_{j}\right]$ is an r-region of $G$ for some $r$ with $r \geq i_{j},(1 \leq j \leq m)$, and such that at least one component $H$ in $G\left[E_{j}\right]$ is an $i_{j}$-region 
of $G$;

(b) if $H$ is a subgraph of $G$ with $\tau(H) \geq i_{j}$, then $E(H) \subseteq E_{j}$;

(c) the integer $m$ and the sequence of edge subsets are uniquely determined by $G$.

Lemma 8.2.5 Let $k \geq 1$ be an integer, $G$ be a graph with $\bar{\tau}(G) \geq k$. Then each of the following statements holds.

(i) The graph $G$ has a unique edge subset $X_{k} \subseteq E(G)$, such that every component $H$ of $G\left[X_{k}\right]$ is a $\mathcal{T}_{k}$-maximal subgraph. In particular, $G \notin \mathcal{T}_{k}$ if and only if $E(G) \neq X_{k}$.

(ii) If $G \notin \mathcal{T}_{k}$, then $G / X_{k}$ contains no nontrivial subgraph $H^{\prime}$ with $\tau\left(H^{\prime}\right) \geq k .\left(G / X_{k}\right.$ is called the $(\tau \geq k)$-reduction of $G)$.

(iii) If $G \notin \mathcal{T}_{k}$, then $d\left(H^{\prime}\right)<k$ for any nontrivial subgraph $H^{\prime}$ of $G / X_{k}$.

Proof. If $G \in \mathcal{T}_{k}$, then $X_{k}=E(G)$. Hence we assume that $G \notin \mathcal{T}_{k}$. Since $\tau(G)<k \leq$ $\bar{\tau}(G)$, there exists an integer $j$ such that $i_{j-1}<k \leq i_{j}$ by Theorem 8.2.4 (a). Let $X_{k}=E_{i_{j}}$. Then each component $H$ of $G\left[X_{k}\right]$ is a $\mathcal{T}_{k}$-maximal subgraph. By Theorem 8.2.4 (c), $X_{k}$ is unique. Thus part (i) holds.

To prove part (ii), we argue by contradiction. We assume $G / X_{k}$ contains nontrivial subgraph $H^{\prime}$ with $\tau\left(H^{\prime}\right) \geq k$ and $V\left(H^{\prime}\right)=\left\{v_{1}, v_{2}, \cdots, v_{h}\right\}$ with $h \geq 2$. Without loss of generality, suppose the pre-image of $v_{i}$ in $G$ is $H_{i}$, and $H_{i}$ is nontrivial for $1 \leq i \leq t$ and is trivial for $t+1 \leq i \leq h$. We will prove that $\tau\left(G^{\prime}\right) \geq k$, where $G^{\prime}=G\left[\cup_{i=1}^{h} V\left(H_{i}\right)\right]$. By induction, if $t=1$, then $G^{\prime} / H_{1}=H^{\prime}$, and $H^{\prime}, H_{1} \in \mathcal{T}_{k}$. Therefore, $G^{\prime} \in \mathcal{T}_{k}$ by Proposition 8.2.1 (C3). Assume it's true for all $t \leq s$. For $t=s+1$, consider $G^{\prime} / H_{s+1}$. Then $G^{\prime} / H_{s+1} \in \mathcal{T}_{k}$ by induction hypothesis. Thus $G^{\prime} \in \mathcal{T}_{k}$ by Proposition 8.2.1 (C3), and so part (ii) holds.

We argue by contradiction to prove (iii). Assume that $d\left(H^{\prime}\right) \geq k$. Then $\left|E\left(H^{\prime}\right)\right| \geq$ $k\left(\left|V\left(H^{\prime}\right)\right|-1\right)$. By Theorem 8.2.2, $H^{\prime}$ has a nontrivial subgraph $H^{\prime \prime}$ such that $H^{\prime \prime} \in \mathcal{T}_{k}$. Note that $H^{\prime \prime}$ is also a nontrivial subgraph of $G / X_{k}$, contrary to part (ii).

Notice that $d(G) \geq k$ implies $\bar{\tau}(G) \geq k$ by Theorem 8.2.2. Therefore if $d(G) \geq k$, then the unique edge subset $X_{k}$ defined in Lemma 8.2.5(i) exists. 
Lemma 8.2.6 Let $G$ be a graph satisfying $d(G) \geq k$ and let $X_{k} \subset E(G)$ be the edge subset defined in Lemma 8.2.5(i). If $G\left[X_{k}\right]$ has at least two components, then for any nontrivial component $H$ of $G\left[X_{k}\right]$, both $d(H) \geq k$, and $G\left[X_{k}\right]$ has at least one component $H$ with $d(H)>k$.

Proof. For any nontrivial component $H$ of $G\left[X_{k}\right]$, by Lemma 8.2.5(i), $H \in \mathcal{T}_{k}$. Thus $|E(H)| \geq k(|V(H)|-1)$, and so $d(H) \geq k$.

Suppose $G\left[X_{k}\right]$ has $c$ components $H_{1}, H_{2}, \cdots, H_{c}$ with $c \geq 2$. By contradiction, assume $d(H)=k$ for any nontrivial component $H$ of $G\left[X_{k}\right]$. Let $x=\left|E(G)-X_{k}\right|$. Then $\left|E\left(H_{i}\right)\right|=k\left(\left|V\left(H_{i}\right)\right|-1\right)$ for any $1 \leq i \leq c$ and $|E(G)|=\sum_{i=1}^{c}\left|E\left(H_{i}\right)\right|+x=\sum_{i=1}^{c}\left(k\left|V\left(H_{i}\right)\right|-k\right)+x=k \sum_{i=1}^{c}\left|V\left(H_{i}\right)\right|-k c+x=k|V(G)|-k c+x$. Therefore, $x=|E(G)|-k|V(G)|+k c \geq k(|V(G)|-1)-k|V(G)|+k c=k(c-1)$.

Let $G^{\prime}=G / G\left[X_{k}\right]$. Then $G^{\prime}$ is a multigraph with $\left|V\left(G^{\prime}\right)\right|=c>1$ and $\left|E\left(G^{\prime}\right)\right|=x$. Therefore, $d\left(G^{\prime}\right) \geq k$, contrary to Lemma 8.2.5 (iii). Hence $G\left[X_{k}\right]$ has at least one component $H_{i}$ such that $d\left(H_{i}\right)>k$.

Let $H_{1}, H_{2}$ be two subgraphs of a graph $G$. Define

$$
E\left(H_{1}, H_{2}\right)=\left\{e=u v \in E(G): u \in V\left(H_{1}\right), v \in V\left(H_{2}\right)\right\} .
$$

Let $\alpha^{\prime}(G)$ denote the size of a maximum matching of $G$ and $\chi^{\prime}(G)$ the edge chromatic number of $G$. Then we have the well-known Vizing Theorem.

Theorem 8.2.7 (Theorem 17.4 of [1]) For any simple graph $G$ on $n$ vertices, $\Delta(G) \leq$ $\chi^{\prime}(G) \leq \Delta(G)+1 \leq n$.

Since the set of edges of each color is a matching of $G$, we have the following observation.

Observation 8.2.8 For any graph $G,|E(G)| \leq \chi^{\prime}(G) \alpha^{\prime}(G)$. 
Lemma 8.2.9 For any simple graph $G$ with $|E(G)| \geq 1, \alpha^{\prime}(G) \geq\left\lceil\frac{\tau(G)}{2}\right\rceil$.

Proof. We argue by induction on $n=|V(G)|$. It is trivial if $n=2$. Assume that lemma holds for smaller $n$ and $n \geq 3$.

Suppose $\tau(G)=k>0$. Then for any $v \in V(G), d(v) \geq k$. Assume first that $G$ has a vertex $v_{0}$ of degree $k$. Let $G^{\prime}=G-v_{0}$. Since $d_{G}\left(v_{0}\right)=k$ and $\tau(G)=k, v_{0}$ is not a cut-vertex of $G$. Therefore, $G^{\prime}$ is connected and $\tau\left(G^{\prime}\right) \geq \tau(G)=k$. By induction,

$$
\alpha^{\prime}(G) \geq \alpha^{\prime}\left(G^{\prime}\right) \geq\left\lceil\frac{k}{2}\right\rceil=\left\lceil\frac{\tau(G)}{2}\right\rceil .
$$

Hence now we assume that $\delta(G) \geq k+1$. Then by Observation 8.2.8 and Theorem 8.2.7,

$$
n \alpha^{\prime}(G) \geq \chi^{\prime}\left(G^{\prime}\right) \alpha^{\prime}(G) \geq|E(G)| \geq \frac{n}{2}(k+1) .
$$

Therefore, $\alpha^{\prime}(G) \geq \frac{k+1}{2} \geq\left\lceil\frac{k}{2}\right\rceil$.

Following the terminology in [17], the strength $\eta(G)$ is defined as

$$
\eta(G)=\min \{d(G / X):|V(X)|<|V(G)|\}
$$

As indicated in Corollary 5 of $[17], \tau(G)=\lfloor\eta(G)\rfloor$.

A subgraph $H$ of $G$ is $\eta$-maximal if for any subgraph $H^{\prime}$ of $G$ that properly contains $H, \eta\left(H^{\prime}\right)<\eta(H)$.

Theorem 8.2.10 (Theorem 6 in [17], Corollary 3.6 in [49]) For any integer $k$ with $d(G) \geq k$, either $E(G)$ is the union of $k$ edge-disjoint spanning trees, or $G$ has a unique edge subset $X$ such that $H=G[X]$ is $\eta$-maximal with $\eta(H)>k$.

For a connected graph $G$ with $\tau(G) \geq k$, define $E_{k}(G)=\{e \in E(G): \tau(G-e) \geq k\}$.

Theorem 8.2.11 (Theorem 4.2 in [49]) Let $G$ be a connected graph with $\tau(G) \geq k$. Then $E_{k}(G)=E(G)$ if and only if $\eta(G)>k$. 
Lemma 8.2.12 Let $G$ be a simple graph and let $X_{k} \subset E(G)$ be the edge subset defined in Lemma 8.2.5(i). If $H^{\prime}$ and $H^{\prime \prime}$ are two components of $G\left(X_{k}\right)$, then each of the following holds.

(i) $\left|E\left(H^{\prime}, H^{\prime \prime}\right)\right|<k$.

(ii) If $d\left(H^{\prime}\right)>k$, then there exists $K \subseteq H^{\prime}$ such that $d(K)>k$ and $\tau(K-e) \geq k$ for any $e \in E(K)$.

(iii) If $d\left(H^{\prime}\right)>k$, then there exists $e^{\prime} \in E\left(H^{\prime}\right)$ such that $\tau\left(H^{\prime}-e^{\prime}\right) \geq k$, and $E(G)-X_{k}$ has at most one edge joining the ends of $e^{\prime}$ to $H^{\prime \prime}$.

Proof. By Lemma 8.2.5(i), both $H^{\prime}$ and $H^{\prime \prime}$ are $\mathcal{T}_{k}$-maximal subgraphs of $G$.

Let $v^{\prime}, v^{\prime \prime}$ denote the two vertices in $G /\left(H^{\prime} \cup H^{\prime \prime}\right)$ onto which $H^{\prime}$ and $H^{\prime \prime}$ are contracted, respectively. Let $G^{\prime}=G\left[V\left(H^{\prime}\right) \cup V\left(H^{\prime \prime}\right)\right]$. If $\left|E\left(H^{\prime}, H^{\prime \prime}\right)\right|=h \geq k$, then $L^{\prime}=G^{\prime} /\left(H^{\prime} \cup\right.$ $\left.H^{\prime \prime}\right)\left[\left\{v^{\prime}, v^{\prime \prime}\right\}\right] \cong h K_{2} \in \mathcal{T}_{k}$. As $H^{\prime}, L^{\prime} \in \mathcal{T}_{k}$, it follows by Proposition 8.2.1(C3) that $G^{\prime} / H^{\prime \prime} \in \mathcal{T}_{k}$. Note that $H^{\prime \prime} \in \mathcal{T}_{k}$, it follows by Proposition 8.2.1(C3) again that $G^{\prime}=$ $G\left[V\left(H^{\prime}\right) \cup V\left(H^{\prime \prime}\right)\right] \in \mathcal{T}_{k}$, contrary to the assumption that $H^{\prime}$ and $H^{\prime \prime}$ are $\mathcal{T}_{k}$-maximal subgraphs of $G$. Hence we must have $\left|E\left(H^{\prime}, H^{\prime \prime}\right)\right|<k$, and so (i) follows.

Part (ii) follows from Theorems 8.2.10 and 8.2.11 directly.

By Lemma 8.2.9 and part (ii), $\alpha^{\prime}(K) \geq\left\lceil\frac{k}{2}\right\rceil$. Let $M$ be a matching of $K$ of size $\left\lceil\frac{k}{2}\right\rceil$. Then for any $e^{\prime} \in M, K-e^{\prime} \in \mathcal{T}_{k}$ by (ii). Since $e^{\prime} \in E(K),\left(H^{\prime}-e^{\prime}\right) /\left(K-e^{\prime}\right)=H^{\prime} / K$. By Proposition 8.2.1 (C2), $\left(H^{\prime}-e^{\prime}\right) /\left(K-e^{\prime}\right) \in \mathcal{T}_{k}$. Therefore, $H^{\prime}-e^{\prime} \in \mathcal{T}_{k}$ by Proposition 8.2.1 (C3). If for any $e^{\prime} \in M \subset E\left(H^{\prime}\right)$ there are at least two edges joining the ends of $e^{\prime}$ to $H^{\prime \prime}$, then $\left|E\left(H^{\prime}, H^{\prime \prime}\right)\right| \geq\left|E\left(K, H^{\prime \prime}\right)\right| \geq 2\left\lceil\frac{k}{2}\right\rceil \geq k$, contrary to (i). Hence this proves (iii).

Lemma 8.2.13 Let $G$ be a nontrivial graph with $\tau(G) \geq k$. If $d(G)=k$, then for any nontrivial subgraph $H$ of $G, d(H) \leq k$. Moreover, if $\tau(H) \geq k$, then $d(H)=k$.

Proof. Since $\tau(G) \geq k$ and $|E(G)|=k(|V(G)|-1), \tau(G)=k$ and $E(G)$ is a union of $k$ edge-disjoint spanning trees. Let $T_{1}, T_{2}, \cdots, T_{k}$ be edge-disjoint spanning trees of $G$. Then for any nontrivial subgraph $H$ of $G,\left|E(H) \cap E\left(T_{i}\right)\right| \leq|V(H)|-1,1 \leq i \leq k$. 
Therefore,

$$
|E(H)|=\mid E(H) \cap\left(\cup_{i=1}^{k} E\left(T_{i}\right)\left|=\sum_{i=1}^{k}\right| E(H) \cap E\left(T_{i}\right) \mid \leq k(|V(H)|-1) .\right.
$$

Thus $d(H) \leq k$. If $H$ has $k$ edge-disjoint spanning trees, then obviousely $d(H)$ is exactly $k$.

\subsection{Characterizations of Graphic Sequences with Re- alizations Having $k$ edge-disjoint Spanning Trees}

We present the main result of the paper in this section, which is Theorem 1.1 restated here.

Theorem 8.3.1 Let $d=\left(d_{1}, d_{2}, \cdots, d_{n}\right)$ be a nonincreasing graphic sequence. Then $d$ has a realization $G$ in $\mathcal{T}_{k}$ if and only if either $n=1$ and $d_{1}=0$, or $n>1$ and each of the following statements holds.

(i) $d_{n} \geq k$,

(ii) $\sum_{i=1}^{n} d_{i} \geq 2 k(n-1)$.

Proof. The case when $n=1$ is trivial and so we shall assume that $n>1$. If $G \in \mathcal{T}_{k}$, $2 k(|V(G)|-1) \leq 2|E(G)|=\sum_{i=1}^{n} d_{i}$ and each vertex has degree at least $k$. This proves the necessity.

We now prove the sufficiency. Assume $d$ is a nonincreasing graphic sequence satisfying both Theorem 8.3.1 (i) and (ii). We argue by contradiction and assume that

every $d$-realization $G$ is not in $\mathcal{T}_{k}$. 
Suppose $G$ is a $d$-realization. By (8.1), $G \notin \mathcal{T}_{k}$, and so by Lemma 8.2.5 (i), $G$ has a unique edge subset $X_{k} \subseteq E(G)$ such that each component of $G\left[X_{k}\right]$ is a $\mathcal{T}_{k}$-maximal subgraph. Let $X=E(G)-X_{k}$. Since $G \notin \mathcal{T}_{k}, X \neq \varnothing$. Suppose $G-X$ has $c$ components, $H_{1}, H_{2}, \cdots, H_{c}$, which are so labelled that $d\left(H_{1}\right) \geq d\left(H_{2}\right) \geq \cdots \geq d\left(H_{t}\right) \geq k$, and that $H_{j}=K_{1}$ for $j=t+1, \cdots, c$. Define

$$
\mathcal{F}_{1}(G)=\left\{H_{i}: d\left(H_{i}\right)>k\right\} \text { and } \mathcal{F}_{2}(G)=\left\{H_{i}: d\left(H_{i}\right)=k\right\} .
$$

Then $\left|\mathcal{F}_{1}(G)\right|+\left|\mathcal{F}_{2}(G)\right|=t$.

Claim 8.1: If every $d$-realization is not in $\mathcal{T}_{k}$, then there exists a $d$-realization $G$ such that $\left|\mathcal{F}_{1}(G)\right|=1$.

By contradiction, suppose that for any $d$-realization $G,\left|\mathcal{F}_{1}(G)\right| \geq 2$. Choose a $d$ realization $G$ such that

$$
\omega(G-X) \text { is minimized, }
$$

and among all the $d$-realizations $G$ satisfying (8.2), we further choose $G$ so that

$$
|X| \text { is maximized. }
$$

As $\left|\mathcal{F}_{1}(G)\right| \geq 2$, we have $d\left(H_{1}\right), d\left(H_{2}\right)>k$. By Lemma 8.2.12(iii), there exist $e_{1}=u_{1} v_{1} \in E\left(H_{1}\right)$ and $e_{2}=u_{2} v_{2} \in E\left(H_{2}\right)$ such that $H_{1}-e_{1}, H_{2}-e_{2} \in \mathcal{T}_{k}$, and there exists at most one edge in $X$ joining the ends of $e_{1}$ and $e_{2}$. Without loss of generality, assume $u_{1} u_{2}, v_{1} v_{2} \notin E(G)$ and let

$$
G_{1}=\left(G-\left\{u_{1} v_{1}, u_{2} v_{2}\right\}\right) \cup\left\{u_{1} u_{2}, v_{1} v_{2}\right\} \text { and } X_{1}=X \cup\left\{u_{1} u_{2}, v_{1} v_{2}\right\} \text {. }
$$

Then by the choice of these edges $u_{1} u_{2}, v_{1} v_{2}, G_{1}$ is also a $d$-realization. By assumption, $G_{1} \notin \mathcal{T}_{k}$ and $\left|\mathcal{F}_{1}\left(G_{1}\right)\right| \geq 2$. Since $G_{1}-X_{1}=\left(H_{1}-u_{1} v_{1}\right) \cup\left(H_{2}-u_{2} v_{2}\right) \cup H_{3} \cup \cdots \cup H_{c}$ and since each component of $G_{1}-X_{1}$ is in $\mathcal{T}_{k}$, it follows by (8.2) that $X_{1}$ is the unique subset of $E\left(G_{1}\right)$ such that $\omega\left(G_{1}-X_{1}\right)=\omega(G-X)=c$ with each component of $G_{1}-X_{1}$ being a $\mathcal{T}_{k}$-maximal subgraph. Now we have $\left|X_{1}\right|=|X|+2$, contrary to (8.3). Thus Claim 8.1 holds. 
By Lemma 8.2.6, for any graph $G^{\prime}$, either $G^{\prime} \in \mathcal{T}_{k}$ or $\left|\mathcal{F}_{1}\left(G^{\prime}\right)\right| \geq 1$. Now we prove the theorem by contradiction. Suppose for every $d$-realization $G, G \notin \mathcal{T}_{k}$. Then by Claim 8.1, there exists $G$ such that $\left|\mathcal{F}_{1}(G)\right|=1$. Thus we can choose a $d$-realization $G$ satisfying

$$
\left|\mathcal{F}_{1}(G)\right|=1 \text { with }\left|V\left(H_{1}\right)\right| \text { maximized. }
$$

And subject to (8.5), we further choose $G$ such that

$$
|X| \text { is maximized. }
$$

We consider the following cases.

Case 1: $t \geq 2$. Thus $H_{2} \neq K_{1}$.

By Lemma 8.2.12 (iii), there exist $e_{1} \in E\left(H_{1}\right), e_{2} \in E\left(H_{2}\right)$ such that there is at most one edge in $G$ joining $e_{1}$ and $e_{2}$ and $H_{1}-e_{1} \in \mathcal{T}_{k}$. Define $G_{1}$ and $X_{1}$ as in (8.4).

Since $d\left(H_{2}-e_{2}\right)<k, H_{2}-e_{2}$ is no longer in $\mathcal{T}_{k}$. Let $\mathcal{T}_{k}$-maximal subgraphs of $G_{1}\left[\left(H_{1}-e_{1}\right) \cup\left(H_{2}-e_{2}\right)\right]$ be $H_{1,2}, H_{2,1}, \cdots, H_{2, t_{2}}$ where $H_{1}-e_{1} \subseteq H_{1,2}$ and $H_{2,1} \cdots H_{2, t_{2}} \subseteq$ $H_{2}-e_{2}$. For each $H_{2, i}$, since $d\left(H_{2}\right)=k$ and $H_{2, i} \subseteq H_{2}$, by Lemma 8.2.13 either $d\left(H_{2, i}\right)=k$ or $H_{2, i}=K_{1}$. Notice that $G /\left(H_{1} \cup H_{2}\right)=G_{1} /\left[\left(H_{1}-e_{1}\right) \cup\left(H_{2}-e_{2}\right)\right]$. Therefore, $H_{1,2}, H_{2,1}, \cdots, H_{2, t_{2}}, H_{3}, \cdots, H_{c}$ are $\mathcal{T}_{k}$-maximal subgraphs of $G_{1}$. By $(8.5)$ and $\mathcal{F}_{1}\left(G_{1}\right)=$ $\left\{H_{1,2}\right\}, H_{1,2}=H_{1}-e_{1}$.

Let $X^{\prime}$ be the edge subset of $G_{1}$ such that $G_{1}-X^{\prime}=H_{1,2} \cup H_{2,1} \cup \cdots \cup H_{2, t_{2}} \cup H_{3} \cup \cdots H_{c}$. Then $X \neq X_{1}$ and $X \subset X_{1} \subset X^{\prime}$, contrary to (8.6).

Case 2: $t=1$, and so $H_{2}=K_{1}$.

In this case, if $c=2$, then by Theorem 8.3.1(i), there must be at least $k$ edges between $H_{1}$ and $H_{2}$. Since $H_{1} \in \mathcal{T}_{k}$, it follows that $G \in \mathcal{T}_{k}$, contrary to (8.1). Hence we must have $c \geq 3$.

For $i \geq 2$, denote $V\left(H_{i}\right)=\left\{x_{i}\right\}$. Note that for any $H_{i}=K_{1}$, there exists an $H_{j}=K_{1}$ such that $e=x_{i} x_{j} \in X$. For otherwise, $x_{i}$ must only be adjacent to the vertices in $H_{1}$. By Theorem 8.3.1 (i), $\left|E\left(H_{i}, H_{1}\right)\right| \geq k$, contrary to Lemma 8.2.12 (i). Without loss of 
generality, we assume $x_{2} x_{3} \in X$. By Lemma 8.2.12 (ii), there exists a nontrivial subgraph $K \subseteq H_{1}$ such that $K-e \in \mathcal{T}_{k}$ for any $e \in E(K)$.

Claim 8.2: There exists $e^{\prime}=u v \in E(K)$ such that $u x_{2}, v x_{3} \notin E(G)$.

In order to present the proof, we define

$$
\begin{aligned}
& B_{1}=\left\{v \in V(K): v x_{2}, v x_{3} \notin E(G)\right\}, \quad B_{2}=\left\{v \in V(K): v x_{2} \in E(G), v x_{3} \notin E(G)\right\}, \\
& B_{3}=\left\{v \in V(K): v x_{2} \notin E(G), v x_{3} \in E(G)\right\}, \quad B_{4}=\left\{v \in V(K): v x_{2}, v x_{3} \in E(G)\right\} .
\end{aligned}
$$

and let $N\left(B_{1}\right)=\left\{v \in V(K): \exists u \in B_{1}\right.$ such that $\left.u v \in E(K)\right\}$. Note that by definition, we have

$$
V(K)=B_{1} \cup B_{2} \cup B_{3} \cup B_{4} .
$$

If $\exists u v \in E(G)$ such that $u \in B_{3}, v \in B_{2}$, then Claim 8.2 holds. Thus we may assume $N\left(B_{2}\right) \cap N\left(B_{3}\right)=\varnothing$. Therefore, $B_{1} \neq \varnothing$. Otherwise, $N\left(B_{2}\right) \cup N\left(B_{3}\right) \subseteq B_{4}$, forcing $\left|B_{4}\right| \geq k$ since $K \in \mathcal{T}_{k}$, for any vertex $v \in K, d_{K}(v) \geq k$, and so $\left|E\left(H_{1}, H_{2}\right)\right| \geq$ $\left|E\left(B_{4}, x_{2}\right)\right|=\left|B_{4}\right| \geq k$, contrary to Lemma 8.2.12 (i). Hence $B_{1} \neq \varnothing$.

If $E\left(G\left[B_{1}\right]\right) \neq \varnothing$, then Claim 8.2 holds. Thus we may also assume that $E\left(G\left[B_{1}\right]\right)=\varnothing$. It follows that $N\left(B_{1}\right) \cap B_{1}=\varnothing$.

Firstly, we shall show that

$$
N\left(B_{1}\right) \cap\left[B_{2} \cup B_{3}\right] \neq \varnothing .
$$

If (8.8) fails, then by (8.7), $N\left(B_{1}\right) \subseteq B_{4}$. Therefore, $\left|B_{4}\right| \geq\left|N\left(B_{1}\right)\right| \geq k$. But then by definition of $B_{4},\left|E\left(H_{1}, H_{2}\right)\right| \geq\left|E\left(B_{4}, x_{2}\right)\right|=\left|B_{4}\right| \geq k$, contrary to Lemma 8.2.12 (i). This verifies (8.8).

By (8.8), we first assume that there exists $v \in N\left(B_{1}\right) \cap B_{2}$. Thus there exists $u \in B_{1}$ such that $u v \in E(K)$. By the definitions of $B_{2}$ and $B_{1}$, both $v x_{3} \notin E(G)$ and $u x_{2} \notin E(G)$, and so Claim 8.2 follows.

Next, we assume that there exists $u \in N\left(B_{1}\right) \cap B_{3}$. Thus there exists $v \in B_{1}$ such that $u v \in E(K)$. By the definitions of $B_{3}$ and $B_{1}, u x_{2} \notin E(G)$ and $v x_{3} \notin E(G)$. Thus, Claim 8.2 must hold. This completes the proof for Claim 8.2. 
By Claim 8.2, define

$$
G_{2}=\left(G-x_{2} x_{3}-u v\right) \cup\left\{u x_{2}, v x_{3}\right\} \text { and } X_{2}=X-x_{2} x_{3} \cup\left\{u x_{2}, v x_{3}\right\} .
$$

Then by the choice of $u, v, x_{2}$ and $x_{3}, G_{2}$ is also a $d$-realization. We shall show that $\left|\mathcal{F}_{1}\left(G_{2}\right)\right|=1$. Assume, to the contrary, that $\left|\mathcal{F}_{1}\left(G_{2}\right)\right| \geq 2$. Then there exists $S \in \mathcal{F}_{1}\left(G_{2}\right)$ and $S \neq H_{1}-u v$. By Proposition 8.2.1 (C4), $V(S) \cap V\left(H_{1}\right)=\varnothing$. But then $S$ is a subgraph of $G$ other than $H_{1}$, contrary to the assumption that $\left|\mathcal{F}_{1}(G)\right|=1$.

By (8.5), $H_{1}-u v$ is a $\mathcal{T}_{k}$-maximal subgraph of $G_{2}$. Since $G_{2}\left[H_{2} \cup \cdots \cup H_{c}\right]=G\left[H_{2} \cup\right.$ $\left.\cdots \cup H_{c}\right]-x_{2} x_{3}, H_{2}, \cdots, H_{c}$ are $\mathcal{T}_{k}$-maximal subgraphs of $G_{2}$. But now $\left|X_{2}\right|=\left|X_{1}\right|+1$, contrary to (8.6).

This completes the proof of the theorem. 


\section{Chapter 9}

\section{Emergence of Specialization in a Swarm of Robots}

\subsection{Introduction}

In a robotic swarm, heterogeneity may be quantified in terms of diversity, or the variability of the properties of individual agents. Heterogeneity may also involve the specialization of individuals for certain tasks. This collective adaptation strategy is often seen in biology [58]. The design of heterogeneous swarms requires ways to quantify the degrees of heterogeneity and specialization as well as their impact on collective performance. Early work on heterogeneity and specialization in robot teams established methods for the composition of group level behaviors [59, 60] and proposed a measure for heterogeneity [61].

The stick-pulling problem was originally formulated [56] to explore the swarm intelligence paradigm [62] in a context where collaboration is realized through local interactions, with limited or no global communication. The basic task, finding and pulling randomly distributed sticks, requires two robots in asymmetric roles. A robot that finds a stick must wait for another one to help pull the stick. The gripping or waiting time parameter (WTP) $[57,56]$ of a robot is the time it will wait for help before releasing a stick. 
In the original study [56], Ijspeert et al. found that this asymmetric task could benefit from specialization. Through experimentation and a two-level modeling approach, they identified an optimal WTP for a homogeneous swarm. For a heterogeneous swarm with two subgroups (castes or species) of agents, each with a different WTP, they found a family of high-performance pairs of WTP values. This type of heterogeneity led to better performance when the number of robots was less than the number of sticks and did not make a significant difference otherwise.

Li, Martinoli and Mustafa [63] investigated how specialization could be learned by the stick-pulling team. In their system, agents changed their WTP based on local or global reinforcement signals. Learning resulted in optimal performance accompanied by increases in information-theoretic measures of diversity and specialization. This work strengthened the correlation between group performance and diversity and provided an example of global performance improvement through individual adaptation. However, it left open the question whether distinct groups with specialized behaviors could emerge through individual adaptation.

We investigated the advantages of specialization in a slightly modified version of the stick pulling problem[57], using a methodology developed for task allocation [64]. The starting point of our modeling approach was similar to the probabilistic model of [56]. Our higher level of abstraction resulted in a concise and transparent analytical model and in the possibility of scaling simulations into the range of thousands of agents and millions of updates. We identified a maximal performance level that may not be exceeded for any WTP configuration, and showed that it could be reached in many different configurations. Comparing homogeneous and two-species configurations, we showed analytically and confirmed through simulations that the two-species swarm performed better under non-ideal circumstances than the homogeneous one (in the case with more sticks than robots). Echoing the results of [56], we found that specialization was advantageous.

In this work we expand the analysis of optimal configurations and explore collective adaptation based on individual adjustment of the agents. We investigate adaptation strategies from two perspectives: (1) convergence to optimal performance; (2) emergence of subgroups with specialized behaviors. We implement a distributed adaptation algorithm where robots randomly change their WTP with a frequency based on their own 
performance. In the second algorithm we add an exchange mechanism where WTPs of successful agents are assigned to underperforming ones. Both algorithms converge to configurations that ensure optimal performance. The WTP exchange mechanism increases the cohesion of the WTP distribution, causing the system to converge to bounded uni- or bimodal distributions.

\subsection{Model and Analytical Results}

\subsubsection{The Stick Pulling Problem}

$N$ are robots tasked with pulling sticks from the ground. The $S_{T}$ sticks are randomly distributed in the workspace. Two robots are required to pull a stick. Robot behaviors are sketched in Figure 9.1. Robots initially wander in search of sticks and can discover sticks in their immediate vicinity. When a robot finds a stick held by another robot, the robots pull the stick together. If a robot finds a free stick, it holds it waiting for another robot to come along, but will release it after a certain time. We model the discovery of sticks as a stochastic process, characterized by a discovery rate $k_{D}$, the same for all sticks, whether or not they are held by another robot. This rate accounts for all physical and technological constraints, such as: the physical density of sticks, the size and accessibility of the area, the movement and detection capabilities of the robots. The numbers of sticks and robots are constant. The only element that can be chosen by design is the behavior of the robots upon discovery of a free stick. Release after waiting is described as a Poisson process whose characteristic time is the waiting time parameter (WTP) $\tau_{i}$, set individually for each agent.

\subsubsection{Equations of Motion}

The $N$ robots are subdivided into $p \leq N$ groups; $N_{i}$ is the number of agents in group $i$. There are $S_{T}$ sticks in total. At any time, a robot may be free (wandering), or holding a stick. We denote the number of free robots of type $i$ with $F_{i}$, and by $H_{i}$ the number 


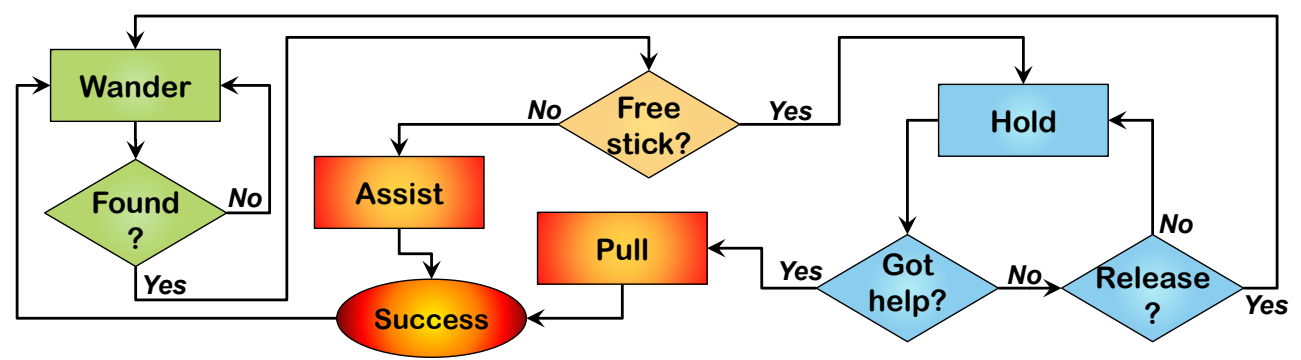

Figure 9.1: Flow chart of robot behaviors in the stick pulling model.

of those holding a stick. The total number of free sticks and free and holding robots are denoted by $S, F$, and $H$. If total number robots of each type are fixed, we have:

$$
\begin{aligned}
N & =\sum_{i=1}^{p} N_{i} ; H=\sum_{i=1}^{p} H_{i} ; F=\sum_{i=1}^{p} F_{i} \\
S_{T} & =S+H \quad ; \quad N=F+H \quad ; \quad N_{i}=F_{i}+H_{i}, \quad \forall i \in\{1, \cdots, p\}
\end{aligned}
$$

Robots in a group have the same WTP, $\tau_{i}$, the average time a robot holds on to a stick before releasing it. The release is controlled by a Poisson process whose rate is $\lambda_{i}=1 / \tau_{i}$. Similarly, the process of discovery of sticks by free robots is characterized by the discovery rate $k_{D}$. Due to (9.1), the state of the system is defined by the number of robots of each type holding a stick, $\left\{H_{1}, \cdots, H_{p}\right\}$. We will write our equations in terms of these variables. Since we are interested in large swarms, we will adopt a continuum approach in describing the dynamics of the system [64].

There are three processes that contribute to the variation of $H_{i}$, capture (discovery), pull, and release of sticks. The capture rate $r_{c a p t}^{(i)}$ is proportional to the number of free robots of type $i$ and the number of free sticks. The pulling rate $r_{\text {pull }}^{(i, j)}$ is proportional to the number of free robots of type $i$ and the number of robots of type $j$ holding a stick. These two processes have the same rate constant, $k_{D}$. Note that the pulling rate does not impact the number of free robots of the type of the second participant. By contrast, the robot that was holding the stick changes its state from holding to free. We denote by $r_{p u l l}^{(i)}$ the total rate of successful pulls of sticks held by robots of type $i$. The release rate $r_{\text {release }}^{(i)}$ of sticks by robots of type $i$ is proportional to the number of robots of type $i$ holding a 
stick, and the rate constant $\lambda_{i}=1 / \tau_{i}$.

$$
\begin{array}{ll}
r_{\text {capt }}^{(i)}=k_{D} F_{i} S=k_{D}\left(N_{i}-H_{i}\right)\left(S_{T}-H\right) & ; \quad r_{\text {pull }}^{(i, j)}=k_{D} F_{i} H_{j}=k_{D}\left(N_{i}-H_{i}\right) H_{j} \\
r_{\text {release }}^{(i)}=\lambda_{i} H_{i} & ; \quad r_{\text {pull }}^{(j)}=k_{D}(N-H) H_{j} .
\end{array}
$$

The net rate of change in $H_{i}$ is then:

$$
\frac{d H_{i}}{d t}=k_{D}\left[\left(N_{i}-H_{i}\right)\left(S_{T}-H\right)-H_{i}(N-H)\right]-\lambda_{i} H_{i}
$$

\subsubsection{Steady state analysis}

We are interested in the steady-state(s) of (9.3). For any such configuration, the righthand side of the equations of motion must vanish. Setting $\frac{d H_{i}}{d t}=0$, we have:

$$
k_{D}\left[N_{i}\left(S_{T}-H\right)-H_{i}\left(S_{T}+N-2 H\right)\right]=\lambda_{i} H_{i}
$$

This equilibrium condition is more transparent in terms of dimensionless variables:

$$
\frac{S_{T}}{N} \equiv \sigma ; \frac{H}{N} \equiv \phi ; \frac{H_{i}}{N_{i}} \equiv \varphi_{i} ; \frac{N_{i}}{N} \equiv \rho_{i} \longrightarrow \varphi_{i}=\frac{\sigma-\phi}{\xi_{i}+(1+\sigma-2 \phi)} .
$$

The dimensionless time parameter $\xi_{i}$ is the ratio between the average time between two discoveries of the same stick by two robots, and the waiting time parameter $\tau_{i}$ :

$$
\xi_{i} \equiv \frac{\lambda_{i}}{N k_{D}}=\frac{1}{N k_{D} \tau_{i}}=\frac{1 /\left(N k_{D}\right)}{\tau_{i}} .
$$

The occupancy fraction $\phi$ is a weighted average of the individual occupancies $\varphi_{i}$. Substituting the individual equilibrium conditions, we arrive at a global condition:

$$
\phi \equiv \frac{H}{N}=\sum_{i} \rho_{i} \varphi_{i} \longrightarrow \phi=\sum_{i} \frac{(\sigma-\phi) \rho_{i}}{\xi_{i}+(1+\sigma-2 \phi)}=f(\phi) .
$$

It can be shown that the equation $\phi=f(\phi)$ has a unique solution, which corresponds to a stable equilibrium of the equations of motion (9.3). 


\subsubsection{Pulling rates and optimality}

The global pulling rate, (given $N$ robots of which $H$ are holding sticks) is

$$
R_{\text {pull }}=k_{D}(N-H) H .
$$

Since $0 \leq H \leq N, R_{\text {pull }}$ is always positive, vanishes for $H=0$ and $H=N$, and is maximal for $H=H^{*} \equiv \min \left(N / 2, S_{T}\right)$. The maximal pulling rate is $R_{\text {pull }}^{*}$ below:

$$
R_{\text {pull }}^{*}=k_{D}\left(N-H^{*}\right) H^{*} ; \quad R_{\text {pull }}^{*}\left(N, S_{T}\right) \leq R_{\text {pull }}^{\max }(N) \equiv \frac{1}{4} k_{D} N^{2} .
$$

Here, $R_{\text {pull }}^{\max }$ is the maximal pulling rate for $N$ robots; it may be achieved if there are enough sticks $\left(S_{T}>N / 2\right)$. If the number of robots $N$ is larger than $2 S_{T}$, the maximal pulling rate is limited to $k_{D}\left(N-S_{T}\right) S_{T}$. We will assume $N<2 S_{T}$, so $R_{\text {pull }}^{*}=R_{\text {pull }}^{\max }$.

\section{Optimal WTP configurations}

The objective of designing our swarm is to maintain the system performance as close to the ideal situation $H=N / 2$ as possible. For a given configuration of groups and WTPs, we can calculate the equlibrium state of the system and the corresponding pulling rate, by solving the equilibrium condition (9.7) for the global occupancy $\phi$, and use it to calculate the individual occupancies. We then specify conditions for optimality by requiring $\phi=1 / 2$. A configuration of waiting time parameters that results in $\phi=1 / 2$ is called optimal or ideal.

One species: If all agents have the same WTP $\tau$, the equilibrium condition (9.9) reads

$$
2 \phi^{2}-(2+\sigma+\xi) \phi+\sigma=0 .
$$

Of the two solutions for $\phi$, only one is in the $[0,1]$ interval. The design problem here consists of determining the waiting time parameter $\tau$ (through the dimensionless time parameter $\left.\xi=1 / N k_{D} \tau\right)$ so that optimal performance is achieved. We can calculate the value of the ideal $\xi=1 / N k_{D} \tau$ by substituting $\phi=1 / 2$ :

$$
\xi^{*}=\sigma-1 \leftrightarrow \tau^{*}=\frac{1}{k_{D}\left(S_{T}-N\right)} .
$$


Two types of robots: We have to design three quantities, the two WTPs $\tau_{1}, \tau_{2}$, and the ratio $\rho_{1} / \rho_{2}$ between the sizes of the groups. Given $\left\{\xi_{1}, \xi_{2}, \rho_{1}, \rho_{2}\right\}$, the equilibrium configuration is uniquely defined. Choosing $\rho_{1}=\rho_{2}=1 / 2$, we obtain the following constraint for the dimensionless time parameters $\left\{\xi_{1}, \xi_{2}\right\}$ :

$$
\frac{1}{\xi_{1}+\sigma}+\frac{1}{\xi_{2}+\sigma}=\frac{2}{2 \sigma-1}
$$

Equal size groups: Consider a number of $p$ different species, each representing $1 / p$ of the population. ${ }^{1}$ The optimality condition is

$$
\frac{p}{2 \sigma-1}=\sum_{i=1}^{p} \frac{1}{\xi_{i}+\sigma} \longleftrightarrow \frac{1}{p} \sum_{i=1}^{p} \frac{1}{\xi_{i}+\sigma}=\frac{1}{2 \sigma-1}
$$

For $p=1$ we reobtain the condition for the ideal $\xi$. The second version of the condition can be interpreted as a requirement that the average of the quantities $1 /\left(\xi_{i}+\sigma\right)$ match the ideal value $1 /(2 \sigma-1)$.

\section{Robustness Measures}

The optimality requirement (9.13) represents a single algebraic constraint. With $p$ equal sized groups, all but one of the WTPs $\left\{\tau_{1}, \cdots, \tau_{p}\right\}$ may take any value over a semi-infinite interval. The corresponding configurations form a $p-1$ dimensional manifold in the $p$ dimensional space of WTP configurations. We are interested in additional performance criteria to characterize these ideal configurations.

Performance under changing conditions: Consider the pulling rate of a system that is optimal for a stick/robot ratio of $\sigma_{0}$, when faced with a different $\sigma \neq \sigma_{0}$. In Figure 9.2 we compare a one-group configuration with $\tau=\tau^{*}$ (9.11) and two configurations of two groups of equal size with WTP pairs $\left\{\tau_{1}, \tau_{2}\right\}$ that satisfy $(9.12)$, for $\sigma=\sigma_{0}=10$. The larger $\tau_{1}$, the smaller $\tau_{2}$ has to be. The factor $K=\tau_{1} / \tau^{*}$ is a measure of how far the $\left\{\tau_{1}, \tau_{2}\right\}$ pair is from the one-species case $(K=1)$. Theoretical predictions for the pulling rate for $K=1,10,100$ are confirmed by simulation results as indicated. The

\footnotetext{
${ }^{1}$ This also applies to the situation when the robots are essentially independent, taking $p=N$.
} 


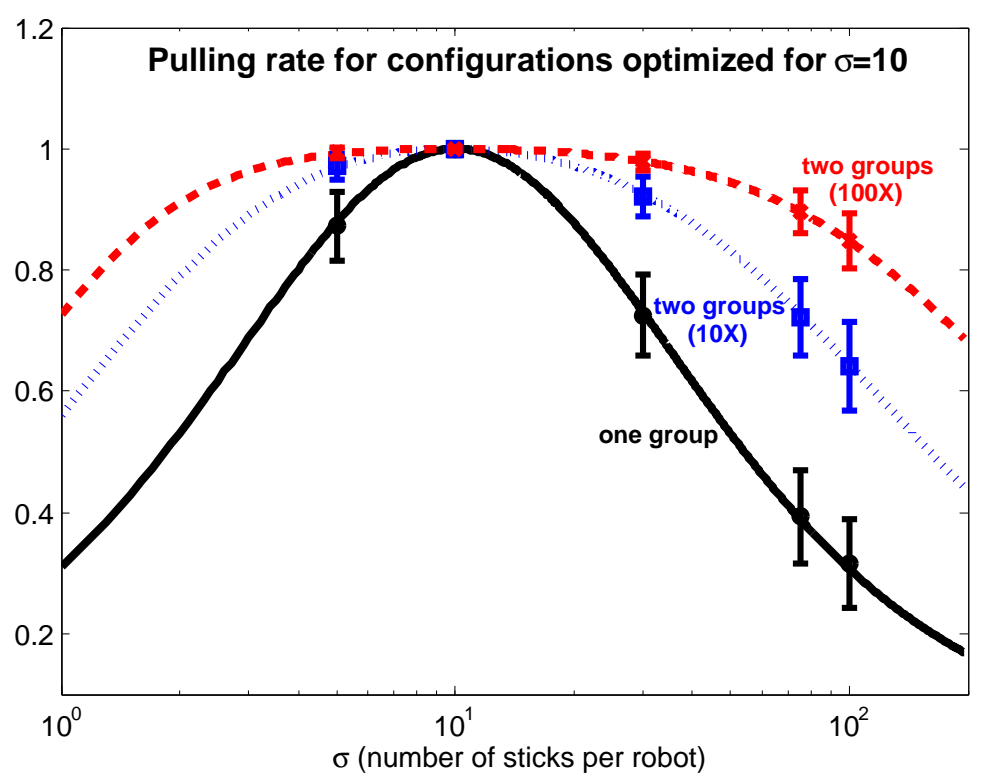

Figure 9.2: Efficiency loss when conditions differ from ideal. Theoretical predictions (lines) and simulation results (points) for the pulling rate of a one-group and several two-group configurations that are optimal for $\sigma=10$. The WTP pairs $\left\{\tau_{1}, \tau_{2}\right\}$ of the two-group configurations satisfy (9.12), with the stated values of the ratio $K=\tau_{1} / \tau^{*}$ (10 and 100, respectively). The simulations (one per data point) had $N=150$ robots and numbers of sticks so that $\sigma=S_{T} / N=5,10,30,75,100$. 
loss of performace is the strongest for the one-group configuration $(K=1)$ and becomes milder as the ratio between $\tau_{1}$ and $\tau^{*}$ increases.

Loss of agents: As a measure of how much of the optimality would be preserved by a subset of the agents in a given configuration, it is useful to compare the pulling efficiency per agent for a configuration where some agents are destroyed. This measure is relevant when comparing WTP configurations that result from randomized adaptation algorithms, where no two agents would likely have the same WTP. It provides a mechanism to penalize configurations that are "too heterogeneous".

\subsection{Simulation Methods}

\subsubsection{Basic simulation algorithm}

The main simulation algorithm is derived from the Gillespie algorithm used in biochemistry and adapted to multi-robot systems in previous work [57]. The state of the system is defined by that of the individual agents. Each of the $N$ agents can be free $(F)$ or holding a stick $(H)$. There are three possbile transitions, corresponding to the three processes discussed above:

$$
F_{i}+S \rightarrow H_{i}(\text { CAPture }) ; H_{i}+F_{j} \rightarrow F_{i}+F_{j}+S(\mathrm{PULl}) ; H_{i} \rightarrow F_{i}+S \text { (RELEASe) }
$$

In the CAPTURE $(i)$ process, agent $i$ goes from Free to Holding; the reverse is the RELEASE $(i)$ process. In the $\operatorname{PULL}(i, j)$ process, agent $i$ goes from holding to free, but the process requires another agent $(j)$, whose state is not ultimately changed. Transitions are controlled by independent Poisson processes; the probability per unit time (or rate) for a specific transition is given by a time constant and the number of eligible partners, if applicable. For example, if both agents $i$ and $j$ are free, the probability per unit time for capturing a stick is the same for both of them, $k_{D} S$. The release rate for agent $i$ while holding a stick is $\lambda_{i}=1 / \tau_{i}$.

In the Gillespie algorithm, simultaneous Poisson processes are simulated by generating next event times for each process, then implementing the state transition that corresponds 
to the smallest one of the next event times. When there are many possible transitions, one may calculate the cumulative transition rate for each type of transition, then choose a specific (pair of) agent(s) for the transition. This approach is correct for simultaneous Poisson processes and we do this for events that involve encounters between free robots and sticks. The additional computational cost due to updating the states of individual agents is almost negligible. Thus, the Poisson model for transitions allows us to have the equivalent of an agent-based simulation for the cost of a centralized one.

\subsubsection{Individual adaptation}

We construct a self-evaluation measure or satisfaction level $\chi_{i}$ for agent $i$, as follows. Every time agent $i$ participates in a successful pull (in either role), $\chi_{i}$ is incremented by 1 . At every update, $\chi_{i}$ decreases exponentially with a characteristic time $\tau_{\text {forget }}$ : $\chi_{i}(t+\Delta t)=\chi_{i}(t) \exp \left(-\Delta t / \tau_{\text {forget }}\right)$. Thus, agents have a memory of past successes, but their satisfaction level decreases as they go through a dry spell.

The satisfaction level defined here does not provide an absolute measure of an individual agent's effectiveness. There is no reference value for it, unless the agent knows what pulling rate it should expect. The maximal pulling rate can be computed from the number of sticks and agents; however, we are interested in an adaptive strategy that can find the optimum without relying on global knowledge.

In this algorithm, each agent changes their WTP randomly, at a rate proportional to the inverse of the agent's satisfaction level (lower satisfaction increases the rate of change). Adaptation is implemented as a Poisson process with time constant $\tau_{\text {learn }} / \chi_{i}$, that runs in parallel with the other transitions (but much slower). Every time this process fires, the respective agent changes its WTP with a small random quantity: $\left.\tau_{i}^{\prime}=\tau_{i} \exp ((r-1 / 2) \Delta)\right)$ where $r$ is a uniformly distributed random number between $[0,1]$ and $\Delta$ is the MonteCarlo $(M C)$ step size (typically a small number). This algorithm results in a random walk in the space of $\log \left(\tau_{i}\right)$ biased by the satisfaction function. Our approach is simpler than the one used by Li et al., but it also relies on a proper self-assessment of performance. 


\subsubsection{Swapping}

As we discuss next, the individual adaptive strategy succeeds in optimizing the pulling rate, but generates configurations where the individual WTPs are spread over many orders of magnitude. In order to increase the coherence of the resulting WTP distributions, we introduced a collective mechanism to supplement individual adaptation. It consists of an additional WTP change, performed with a small (fixed) probability $\nu$, during normal WT$\mathrm{P}$ updates. This corresponds to an additional Poisson process, with propensity $\nu / \tau_{\text {learn }}$. When this process fires, we select a pair of agents, a donor (with a high satisfaction level), and an acceptor (with a low satisfaction level), and change the WTP of the acceptor to that of the donor. This procedure is reminiscent of biologically inspired algorithms. While it requires some degree of collective communication, it can be implemented in a way that ensures reasonable scaling as the number of agents increases. The key is in that the donor and acceptor agents can self-select and communicate using a pre-determined procedure of asynchronous communication to upload or download their WTPs.

\subsection{Results and Discussion}

\subsubsection{Model Validation - Equilibration}

The standard system in our simulations consists of $N=150$ robots and $S_{T}=2000$ sticks. We use time units where the discovery rate $k_{D}=1$. Thus, the maximal pulling rate is $\frac{1}{4} N^{2} k_{D}=5625$ pulls per unit time. The corresponding optimal waiting time is $\tau^{*}=1 / k_{D}\left(S_{T}-N\right)=5.4 \times 10^{-4}$. The average time between two robot-stick encounters is $\tau_{E}=1 /\left(k_{D} S_{T} N\right)=3.33 \times 10^{-6}$. The time between two consecutive updates in a simulation is on the order of $10^{5}$ iterations per time unit. We performed simulations with various WTP configurations and verified that the system converges to the average occupancy fractions and pulling rates predicted by the continuum equations in Sec.9.2 . The value of the equilibration time is comparable to the average time of $5 \times 10^{-4}$ units it takes for one robot to find one of the 2000 sticks. Figure 9.2 shows theoretical and simulation results for the equilibrium pulling rate for configurations with one or two 
WTP groups, for the same number of robots $(N=150)$, but different numbers of sticks (simulations with $\sigma \equiv S_{T} / N=5,10,30,75,100$ ). All configurations are ideal for $\sigma=10$ $\left(S_{T}=1500\right.$ sticks). The simulation results confirm the analytical predictions given in Sec.9.2.4.

\subsubsection{Individual adaptation algorithm}

We implemented the individual adaptation algorithm described in Sec.3.2 on the $N=150$, $S_{T}=2000$ system, exploring parameter values around $\tau_{\text {learn }}=1.0 \times 10^{-4}, \tau_{\text {forget }}=0.1$ and a Monte-Carlo step size of $\Delta=1.0 \times 10^{-2}$.

The evolution of the system with these parameter values is shown in Figure 9.3. The waiting time parameters are initially set to $50 \%$ of the optimal value $\tau^{*}$. As the individual $\tau$ values change, the number of free robots evolves, reaching the optimum of 75 in approximately 400 time units. This $\tau$-convergence time is significantly longer than the equilibration time of $5 \times 10^{-4}$ it takes the number of free robots to reach the equilibrium value corresponding to a fixed WTP configuration. It is useful to visualize the time evolution of WTPs using the distribution of the $\log (\tau)$ values, as in Figure 9.3. The results are qualitatively different from the one- or the two-group configurations described previously. As the simulation starts, the $\log (\tau)$ distribution spreads out and continues to do so over time, expending into extremely large and small (positive) values, reaching widths of 10 orders of magnitude and higher after $10^{8}$ steps. The $\log (\tau)$ distribution is close to a normal, whose standard deviation increases like the square root of the simulation time.

We performed a number of simulations to investigate the effect of changing the adaptation parameters on convergence. The results are presented in Figure 9.4. We define convergence for the purposes of these simulations as the state (after the initial equilibration) where the moving average over 10,000 iterations of the number of free robots is within 1 of the ideal value of 75 . We limited the simulations to $10^{7}$ iterations, and we plot both the time to convergence and the number of held sticks after the maximum number of iterations. For the converged simulations, the final number of sticks is very close to 

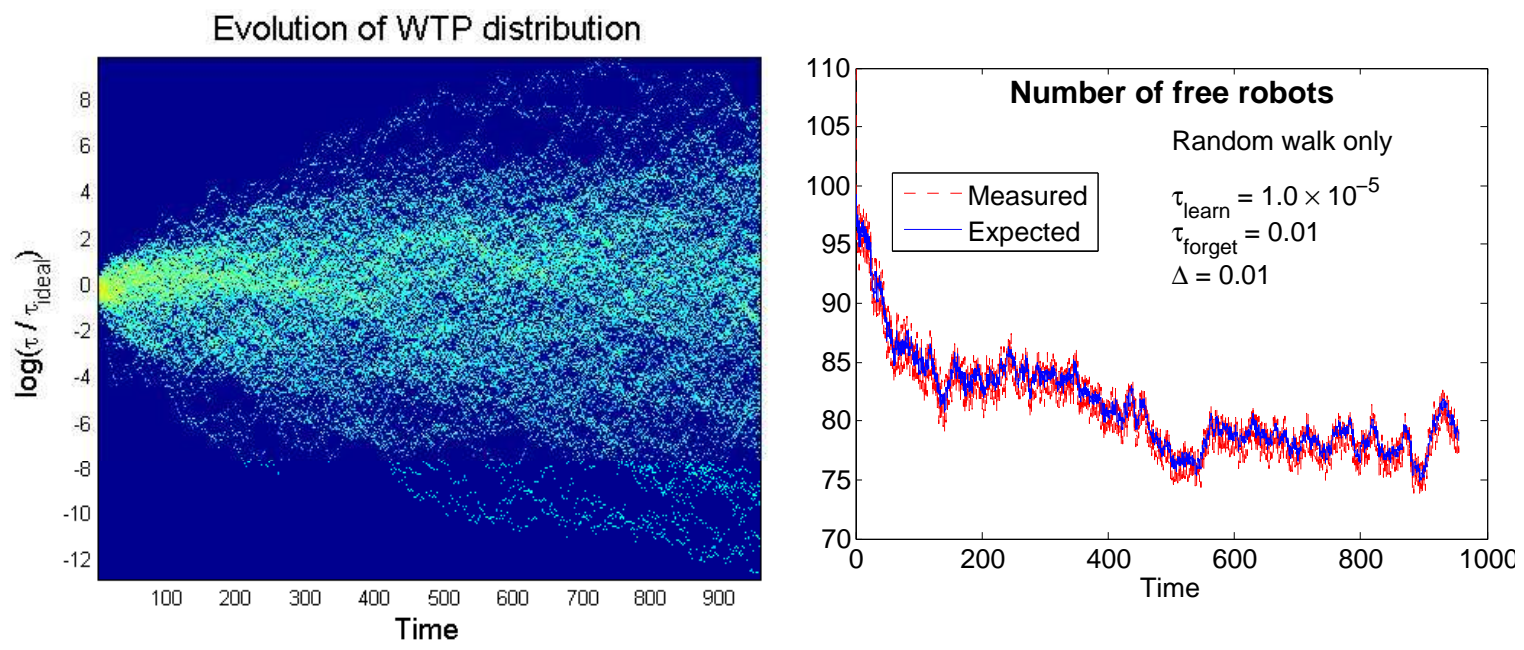

Figure 9.3: (Left) Evolution of the distribution of WTPs in an adaptive simulation. Notice the similarity to a diffusion process. (Right) The number of free robots in the same simulation (expected $=$ based on the current configuration of WTPs). Based on a single simulation with $N=150$ robots, $S_{T}=2000$ sticks, and $3 \times 10^{8}$ updates.

75 , and the convergence time varies. For the unconverged simulations, better adaptation corresponds to final sticks held counts that are closer to the ideal value of 75 .

The dependence on the averaging time $\tau_{\text {forget }}$ is relatively weak. None of the simulations using this algorithm converged (within the iteration limit), due to the values for $\Delta$ and $\tau_{\text {learn }}$. However, the final state approaches 75 as $\tau_{\text {forget }}$ is reduced by a factor of 10 , and moves further away as $\tau_{\text {forget }}$ is increased. Increase in the frequency of WTP changes (decreased $\tau_{\text {learn }}$ ) leads to marginal improvement. A 10-fold increase in the Monte-Carlo step size $\Delta$ improves the adaptation performance to the point where the system converges within the $10^{7}$ iteration cutoff. Further increase of the step size leads to additional improvement in the convergence time. However, the configurations reached in this manner are increasingly incoherent, with a very wide WTP distribution. 

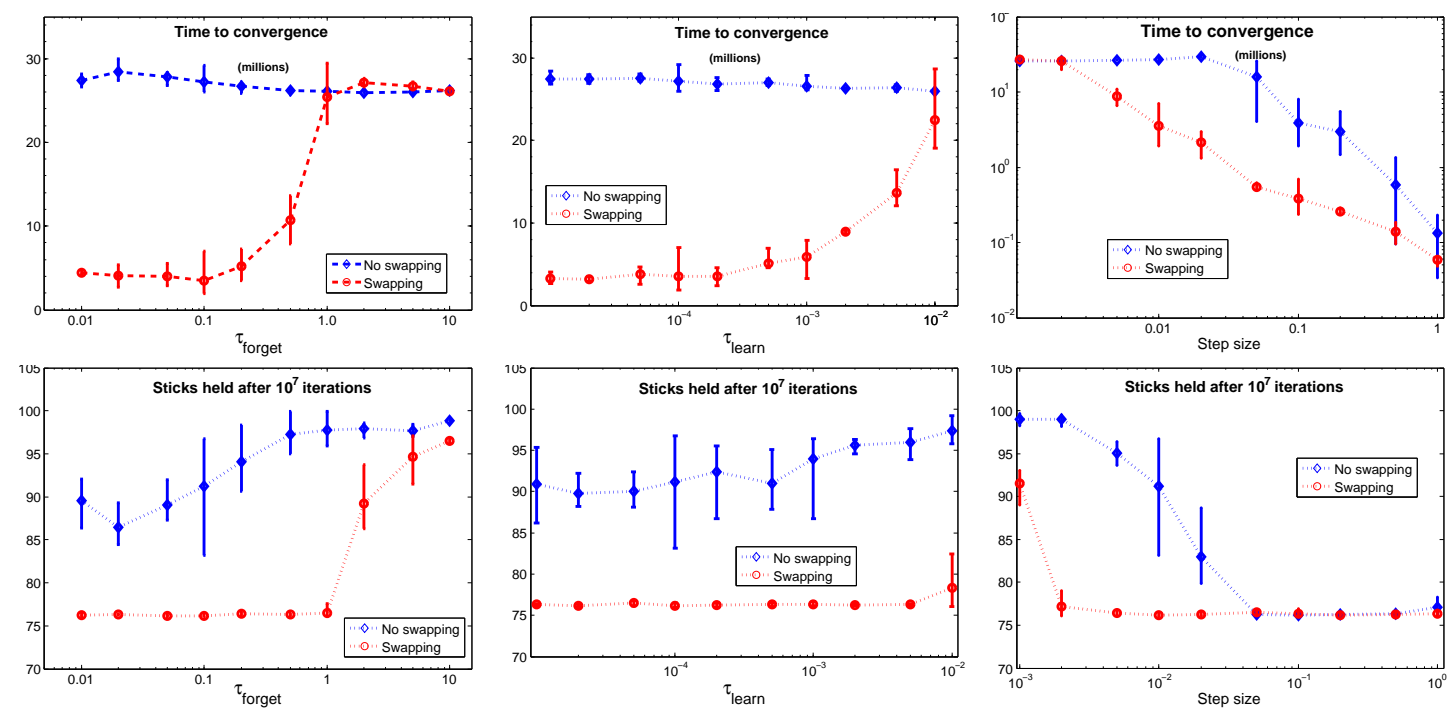

Figure 9.4: Convergence time (top, in millions of iterations) and sticks held after $10^{7}$ iterations (bottom) versus $\tau_{\text {forget }}, \tau_{\text {learn }}$, and MC step size $\Delta$, in the individual adaptive algorithm (blue) and with swapping (red). Each point represents 5-20 simulations with $N=150$ robots and $S_{T}=2000$ sticks. 


\subsubsection{Swapping algorithm}

The introduction of swapping leads to dramatically improved convergence, over all parameter values investigated. It is remarkable that WTP swapping, performed at a frequency corresponding to one swap per every 100 individual WTP changes, improves convergence this much. The parameter sensitivity results for this algorithm are also plotted in Figure 9.4. The effect of parameter changes is qualitatively similar in the two algorithms. The swapping result converges for all but the highest values of the averaging time $\tau_{\text {forget }}$. The performance of the algorithm deteriorates as this parameter is increased, and convergence is lost as $\tau_{\text {forget }}$ goes from 0.1 to 1.0. Increased $\tau_{\text {learn }}$ also reduces the performance of the swapping algorithm.

The first algorithm was the most sensitive to the Monte-Carlo step size $\Delta$. Increased $\Delta$ improved the convergence of both algorithms, and their performance became similar as $\Delta \approx 1$. A value of $\Delta=1$ means that the random change of a WTP is comparable to the value of the WTP. With such large variation steps, the newly selected parameters have little to do with the previous ones. In this limit, the algorithm tends to become purely random selection of parameters rather than a search process (on the level of individual agents).

Swapping dramatically limits the expansion of the WTP distribution, as shown in Figures 9.5 and 9.6. For most parameter values (except very high $\Delta$ ) the simulations resulted in bounded, unimodal distributions with a spread of little more than one order of magnitude, much less than in the individual adaptation case (compare Fig.9.6 and 9.3). In the longer term, some of the simulations exhibit transitions to bimodal distributions. The bimodal distributions we observed had narrow modes, with maxima separated by 1-2 orders of magnitude. While the bimodal distributions extended over almost three orders of magnitude, they remained bounded, and the system eventually transitioned back to the unimodal regime. 

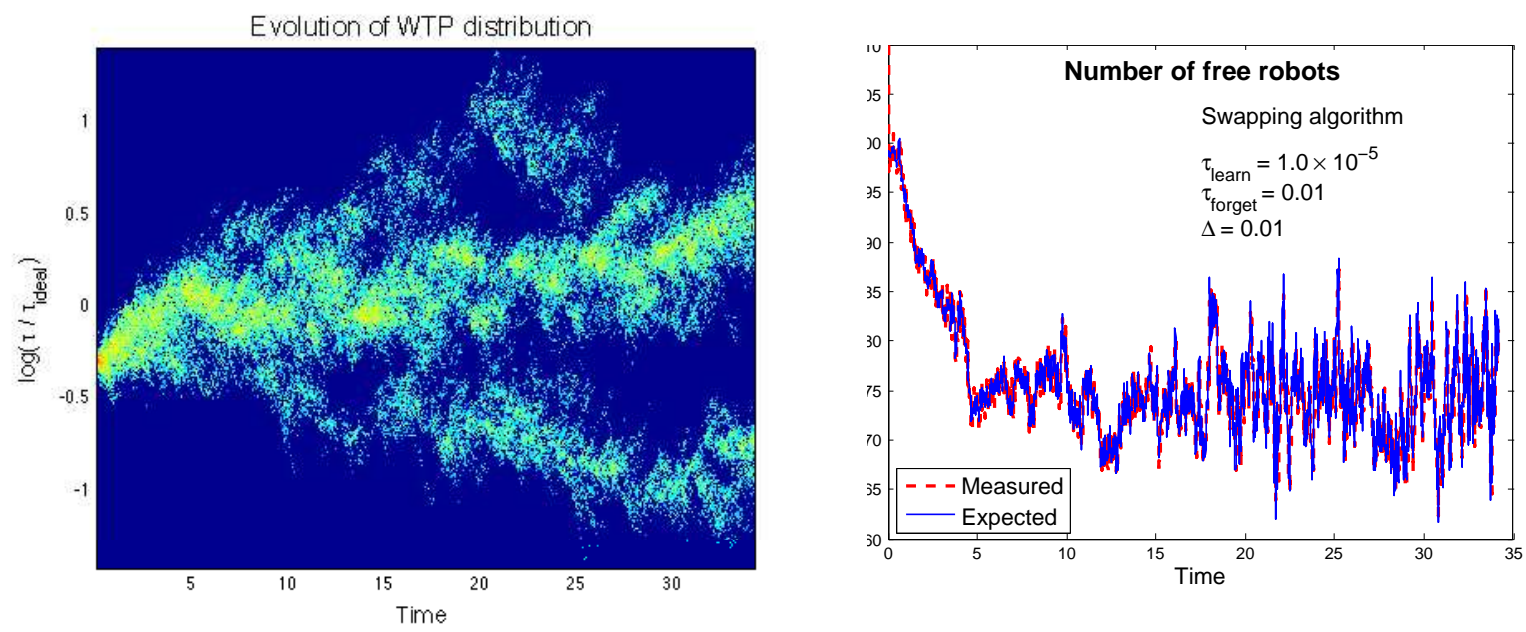

Figure 9.5: Evolution of the distribution of waiting time parameters (left) and the number of free robots (right) for an adaptive simulation with swapping (expected $=$ based on the current configuration of WTPs). Note the different time scale of convergence compared to the non-swapping simulation shown in Figure 9.3; the two simulations have the same adaptation parameters. Based on a single simulation with $N=150$ robots, $S_{T}=2000$ sticks, and $10^{7}$ update steps. 


\subsubsection{Waiting time parameter distributions}

From a design and analysis perspective, configurations with one, two or a small number of distinct waiting time parameters seem more straightforward. By contrast, both adaptation algorithms result in configurations that can only be characterized by a continuous distribution of waiting time parameters, rather than one or a few distinct WTPs shared by groups of agents.

The evolution of the $\log (\tau)$ in the individual adaptation algorithm (Figure 9.3) is similar to pure diffusion, consistent with a random walk. This makes sense because each individual WTP change is a small variation taken from a distribution that is symmetric in terms of $\log (\tau)$. This random walk is influenced through the satisfaction function and is practically confined to globally optimal configurations. The global optimality condition (9.13) corresponds to a single constraint on the $N$ waiting time parameters. If the individual $\log (\tau)$ are allowed to increase or decrease indefinitely, most agents will have waiting times that are either much larger or much smaller than the ideal value. In this case, the $1 /\left(\xi_{i}+\sigma\right)$ terms in Eq.(9.13) would approach $1 / \sigma$ and 0 , respectively. The optimality condition for a configuration with $N_{\text {high }}$ agents with very high WTP $\left(\tau_{i}>>1 \rightarrow \xi_{i}<<1\right.$ ) and the rest with very small WTP is simply

$$
\frac{N_{h i g h}}{N}=\frac{\sigma}{2 \sigma-1}=\frac{S}{2 S-N} .
$$

This simple constraint on the values of the WTPs of the agents in the high and low groups ensures optimality for the late WTP configurations obtained in the individual adaptation algorithm. We will call these divergent-optimal or DO configurations. Presumably, these could also be obtained more easily, by a simple random search on the level of individual agents. A DO configuration can be interpreted as an example of specialization (castes with $\tau=\{0, \infty\})$.

\subsection{Summary and Conclusions}

We have expanded our analysis[57] of the stick-pulling problem and established that each WTP configuration corresponds to a unique equilibrium pulling rate which can be 

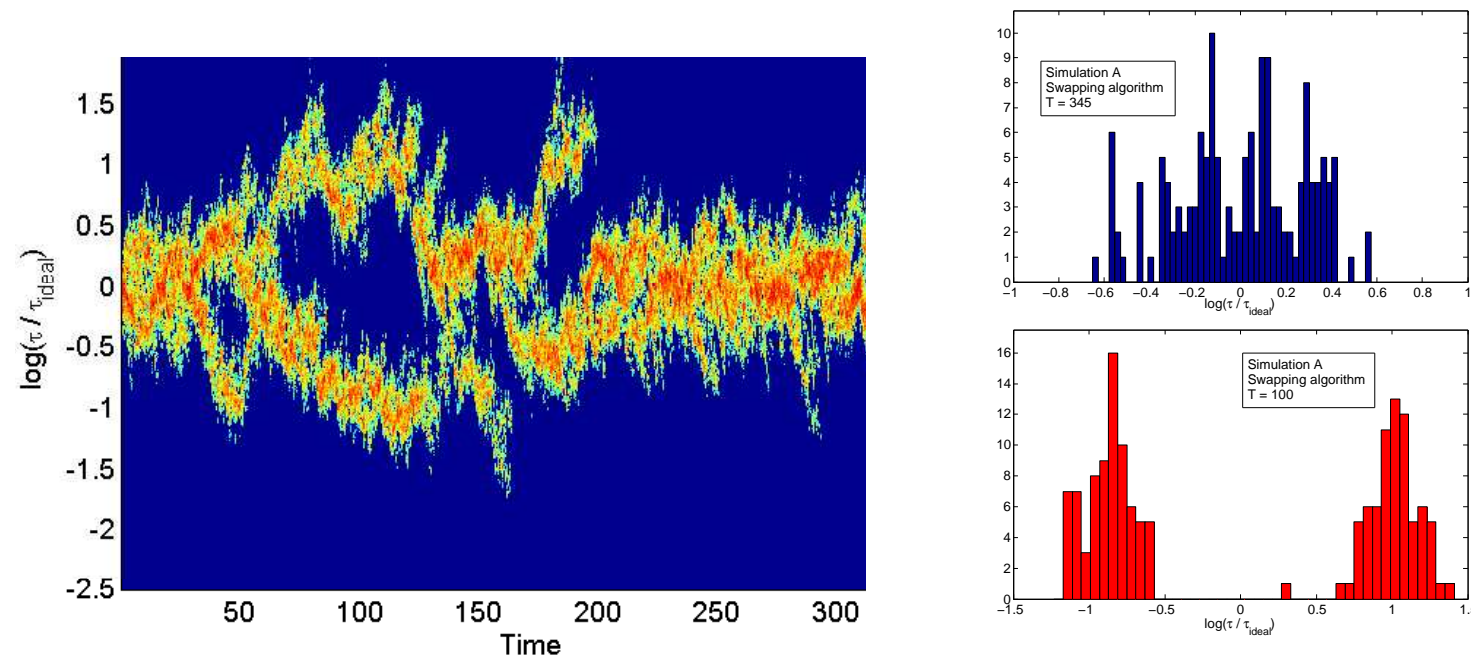

Figure 9.6: Evolution of the distribution of waiting time parameters during a long simulation with swapping. Based on a single simulation with $N=150$ robots, $S_{T}=2000$ sticks, and $10^{8}$ update steps.

estimated analytically. We showed that there is a maximum possible or optimal pulling rate for a given number of sticks and robots (9.9). The optimality requirement can be formulated as a single algebraic condition (9.13) for the $N$ parameters.

We designed and implemented two adaptive optimization strategies and showed that both converge to optimal configurations. The individual adaptation algorithm relies exclusively on the agents' own record of their performance, in the form of a satisfaction function. Robots change their WTP based on this function (low satisfaction $\rightarrow$ higher change rate). Each change is a Monte-Carlo step in a random direction. The evolution of the WTP distribution in this algorithm is consistent with diffusion. The distribution of $\log (\tau)$ approaches a normal whose witdh increases indefinitely, while maintaining optimal performance. The long-term limit for this type of distribution, called divergent-optimal (DO), has WTPs that approach either zero or infinity. Optimality can be ensured by the appropriate ratio between the two groups (9.14). DO configurations can be regarded as extreme examples of emerging specialization. The $\tau \rightarrow \infty$ species specializes in discovering and holding sticks, and the $\tau \rightarrow 0$ specializes in assisting stick holders. 
In the swapping algorithm we supplement individual adaptation with a mechanism that assigns the WTP of well performing agents to under-performing ones. While requiring a limited amount of global communication, this algorithm leads to dramatic improvement of the rate of convergence. It also limits the width of the WTP distributions. Increased Monte-Carlo step size in the swapping algorithm leads to faster converence but eventually results in the emergence of DO configurations.

Emergence of specialization can also be observed in the swapping algorithm, where long-term simulations fluctuate between bounded uni- and bimodal distributions with narrow modes. The bounded bimodal configurations are closer to the idea of specialized groups, each with a narrowly defined set of features (similar to biological phenotypes).

In summary, our results provide two mechanisms by which specialized groups of agents can emerge from an agent-based adaptation strategy. The more easily obtained DO configurations may not be satisfactory for a given application. Further refinements are necessary to stabilize the bounded bimodal configurations. This will require more sophisticated measures of performance, which can enforce our preference for one or another type of WTP distribution. We gave two possible examples of such measures that may be implemented in future applications. Finally, future work in this direction should also integrate results from machine learning and information theory. 


\section{Bibliography}

[1] J.A. Bondy and U.S.R. Murty, Graph Theory with Applications, American Elsevier, New York (1976).

[2] P.A. Catlin, A reduction method to find spanning eulerian subgraph, J. Graph Theory, 12 (1988) 29-45.

[3] P.A. Catlin, Edge-connectivity and edge-disjoint spanning trees, preprint, see: http : //www.math.wvu.edu/ hjlai/Pdf/Catlin_Pdf/Catlin49a.pdf.

[4] P.A. Catlin, Z.Y. Han, H.-J. Lai, Graphs without spanning closed trails, Discrete Math, 160 (1996) 81-91.

[5] P.A. Catlin and H.-J. Lai, Spanning trails jointing two given edges, Graph Theory, Combinatrorics, and Application (vol.1), edited by Y. Alavi, G. Chartrand, O.R. Ollermann, A.J. Schwenk, (1991) 207-222.

[6] F. Harary and C. St. J. A. Nash-Williams, On eulerian and hamiltonian graphs and line graphs, Canad. Math. Bull., 8 (1965) 701-709.

[7] L. Lesniak-Foster and J.E. Williamson, On spanning and dominating circuits in graphs, Can. Math. Bull., 20 (1977) 215-220.

[8] D. Li, H.-J. Lai and M. Zhan, Eulerian subgraphs and Hamilon-connected line graph, Discrete Applied Mathematics, 145 (2005) 422-428.

[9] C. Thomassen, Reflections on graph theory, J. Graph Theory, 10 (1986) 309-324. 
[10] S.M. Zhan, Hamiltonian connectedness of line graphs. Ars Combinatoria, 22 (1986) 89-95.

[11] S.M. Zhan, On hamiltonian line graphs and connectivity, Discrete Math, 89 (1991) 89-95.

[12] F. T. Boesch, C. Suffel, and R. Tindell, The spanning subgraphs of eulerian graphs, J. Graph Theory 1 (1977) 79-84.

[13] H. J. Broersma, L. M. Xiong, A note on minimum degree conditions for supereulerian graphs, Discrete Appl. Math. 120 (2002) 35-43.

[14] P. A. Catlin, Supereulerian Graphs: A survey, J. Graph Theory 16 (1992) 159-163.

[15] P. A. Catlin, Super-Eulerian graphs, collapsible graphs and four-cycles, Congr. Number 58 (1987) 233-246.

[16] P. A. Catlin, X. W. Li, Supereulerian graphs of minimum degree at least 4, J. Adv. Math. 28 (1999) 65-69.

[17] P. A. Catlin, J. W. Grossman, A. M. Hobbs and H.-J. Lai, Fractional arboricity, strength, and principal partitions in graphs and matroids, Discrete Appl. Math. 40 (1992) 285-302.

[18] Z. H. Chen, H.-J. Lai, Reduction techniques for super-Eulerian graphs and related topics-a survey, Combinatorics and graph theory '95, Vol. 1 (Hefei), World Sci. Publishing, River Edge, NJ, 1995 53-69.

[19] Z. H. Chen, H.-J. Lai, Supereulerian graphs and the Petersen graph, Ars Combinatoria 48 (1998) 271-282.

[20] H. Fleischner, "Eulerian Graphs and Related Topics". Part 1, Volume 1. Annals Disc. Math. 45 (North Holland, Amsterdam, 1990).

[21] F. Jager, A note on sub-Eulerian graphs, J. Graph Theory 3 (1979) 91-93.

[22] H.-J. Lai, Contractions and Hamiltonian line graphs, J. Graph Theory 12 (1988) $11-15$. 
[23] H.-J. Lai, Eulerian subgraphs containg given edges, Discrete Math. 230 (2001) 63-69.

[24] X. M. Li, D. X. Li and H.-J. Lai, The Supereulerian Graphs in the Graph Family $C(l, k)$, Discrete Math. 309 (2009) 2937-2942.

[25] C. St. J. A. Nash-Williams, Decompositions of finite graphs into forests. J. London Math. Soc. 39 (1964) 12.

[26] W. R. Pulleyblank, A note on graphs spanned by eulerian graphs, J. Graph Theory 3 (1979) 309-310.

[27] Z. Niu, L. Xiong, Supereulerianity of $k$-edge-connected graphs with a restriction on small bonds, Discrete Appl. Math. 158 (2010) 37-43.

[28] J. Barat and C. Thomassen, Claw-decompositions and Tutte-orientations, J. Graph Theory, 52 (2006) 135-146.

[29] P. A. Catlin, The reduction of graph families closed under contraction, Discrete Math. 160 (1996), 67-80.

[30] P. A. Catlin, A. M. Hobbs and H.-J. Lai, Graph families operations, Discrete Math, 230 (2001), 71-97.

[31] G. A. Dirac, A property of 4-chromatics graphs and some remarks on critical graphs, J. London Math. Soc., 27 (1952), 85-92.

[32] F. Jaeger, On circular flows in graphs, Finite and Infinite Sets, (Eger, 1981), Colloquia Mathematica Societatis Janos Bolyai 37, North Holland, 1984, pp. 391-402.

[33] F. Jaeger, Nowhere-zero Flow Problems, in "Selected Topics in Graph Theory" (L. Beineke and R. Wilson, Eds), Vol. 3 pp91-95. Academic Press, London/New York, 1988.

[34] F. Jaeger, N. Linial, C. Payan and M. Tarsi, Group connectivity of graphs - a nonhomogeneous analogue of nowhere-zero flow properties, J. Combinatorial Theory, Ser. B 56 (1992) 165-182. 
[35] T. Kloks, Treewidth, Computations and Approximations, Springer-Verlag, New York, 1994.

[36] H.-J. Lai, Group connectivity of 3-edge-connected chordal graphs, Graphs and Combinatorics, 16 (2000), 165-176.

[37] H.-J. Lai, Mod $(2 p+1)$-orientations and $K_{1,2 p+1}$-decompositions, SIAM J. of Discrete Math., 21 (2007) 844-850.

[38] H.-J. Lai, Y. H. Shao, H. Wu and J. Zhou, On mod $(2 p+1)$-orientations of graphs, J. of Combinatorial Theory, Series B., 99 (2009), 399-406.

[39] P. D. Seymour, Nowhere-zero 6-flows, J. Combinatorial Theory, Ser. B, 30 (1981) 82-94.

[40] W. T. Tutte, A contribution to the theory of chromatical polynomials, Can. J. Math. 6 (1954), 80-91.

[41] Z.-H. Chen, H.-J. Lai and H. Lai, Nowhere zero flows in line graphs, Discrete Math. 230 (2001), 133-141.

[42] H.-J. Lai, Y. Liang, J. Liu, J. Meng, Y. Shao and Z. Zhang, Contractible graphs with respect to $\bmod (2 p+1)$-orientations, submited.

[43] F. Boesch and F. Harary, Line removal algorithms for graphs and their degree lists, IEEE Transactions on Circuits and Systems, 23 (1976) 778-782.

[44] W. H. Cunningham, Optimal attack and reinforcement of a network. J. Assoc. Comp. Mach. 32 (1985) 549 - 561.

[45] S. Fan, H.-J. Lai, Y. Shao, T. Zhang and J. Zhou, Degree sequence and supereulerian graphs, Disc. Math. 308 (2008), 6626-6631.

[46] S. L. Hakimi, On the Realizability of a Set of Integers as Degrees of the Vertices of a Graph, SIAM J. Appl. Math. 10 (1962), 496-506.

[47] V. Havel, A remark on the existence of fintie graphs (Czech.), Časopis Pěst. Mat. 80 (1955), 477-480. 
[48] H.-J. Lai, P. Li and Y. Liang, Reinforcing matroids with $k$ disjoint bases, Applied Mathematics., 1 (2010), 244-249.

[49] H.-J. Lai, P. Li and Y. Liang, Characterization of removable elements with respect to having $k$ disjoint bases in a matroid, submitted.

[50] J. Li, Degree sequences of graphs, Advances in Mathematics, 23 (1994), 193-204.

[51] D. Liu, H.-J. Lai and Z.-H. Chen, Reinforcing the number of disjoint spanning trees, Ars Combinatoria, 93 (2009), 113-127.

[52] R. Luo, R. Xu, W. Zang, and C.-Q. Zhang, Realizing degree sequences with graphs having nowhere-zero 3-flows, SIAM J. on Discrete Mathematics, 22 (2008) 500-519.

[53] D. Meierling and L. Volkmann, A remark on degree sequences of multigraphs, Math. Meth. Oper. Res. 69 (2009), 369-374.

[54] M. Takahashi, K. Imai and T. Asano, Graphical degree sequence problems, IEICE Trans Fundam E77-A(2):546-552.

[55] X. Yao, X. Li and H.-J. Lai, Degree conditions for group connectivity, Disc. Math., 310 (2010), 1050-1058.

[56] A. Ijspeert, A. Martinoli, A. Billard, and L. M. Gambardella, Collaboration through the Exploitation of Local Interactions in Autonomous Collective Robotics: The Stick Pulling Experiment, Autonomous Robots, vol. 11, no. 2, pp. 149171, 2001.

[57] M. A. Hsieh, A. M. Halasz, E. D. Cubuk, S. Schoenholz, and A. Martinoli, Specialization as an optimal strategy under varying external conditions, in Proceedings of the International Conference on Robotics and Automation (ICRA) 2009, Kobe, Japan, 2009.

[58] E. Kussell and S. Leibler, Phenotypic diversity, population growth, and information in fluctuating environments, Science, vol. 309, no. 5743, pp. 20758, Sep 232005.

[59] M. J. Mataric, Designing and understanding adaptive group behavior, Adaptive Behavior, vol. 4, pp. 5081, 1995. 
[60] K. Lerman, A. Galstyan, A. Martinoli, and A. J. Ijspeert, A Macroscopic Analytical Model of Collaboration in Distributed Robotic Systems, Artificial Life, vol. 7, no. 4, pp. 375393, 2001.

[61] T. Balch, Hierarchic social entropy: An information theoretic measure of robot team diversity, Autonomous Robots, vol. 8, no. 3, pp. 209238, July 2000.

[62] E. Bonabeau, M. Dorigo, and G. Theraulaz, Swarm Intelligence: From natural to artificial systems. New Yok: Oxford University Press, 1999.

[63] L. Li, A. Martinoli, and Y. Abu-Mostafa, Learning and Measuring Specialization in Collaborative Swarm Systems, Adaptive Behavior, vol. 12, no. 34, pp. 199212, 2004, special issue on Mathematics and Algorithms of Social Interactions, C. Anderson and T. Balch (Eds.).

[64] S. Berman, A. Halasz, M. A. Hsieh, and V. Kumar, Optimized stochastic policies for task allocation in swarms of robots, IEEE Transactions on Robotics, vol. 25, no. 4, pp. 927937, 2009. 